University of Massachusetts Amherst

ScholarWorks@UMass Amherst

Chemical Engineering Faculty Publication

Series

2020

\title{
Recent progress in the science of complex coacervation
}

Charles E. Sing

University of Illinois at Urbana-Champaign

Sarah L. Perry

University of Massachusetts Amherst

Follow this and additional works at: https://scholarworks.umass.edu/che_faculty_pubs

\section{Recommended Citation}

Sing, Charles E. and Perry, Sarah L., "Recent progress in the science of complex coacervation" (2020). Soft Matter. 886.

https://doi.org/10.1039/D0SM00001A

This Article is brought to you for free and open access by the Chemical Engineering at ScholarWorks@UMass Amherst. It has been accepted for inclusion in Chemical Engineering Faculty Publication Series by an authorized administrator of ScholarWorks@UMass Amherst. For more information, please contact scholarworks@library.umass.edu. 


\section{Soft Matter}

\section{ARTICLE TYPE}

Cite this: DOI: 00.0000/xxxxxxxxxx

Accepted Date

DOI: $00.0000 / x x x x x x x x x x$

\section{Recent Progress in the Science of Complex Coacerva- tion}

\author{
Charles E. Sing, ${ }^{* a, b}$ and Sarah L. Perry ${ }^{* c, d}$
}

\begin{abstract}
Complex coacervation is an associative, liquid-liquid phase separation that can occur in solutions of oppositely-charged macromolecular species, such as proteins, polymers, and colloids. This process results in a coacervate phase, which is a dense mix of the oppositely-charged components, and a supernatant phase, which is primarily devoid of these same species. First observed almost a century ago, coacervates have since found relevance in a wide range of applications; they are used in personal care and food products, cutting edge biotechnology, and as a motif for materials design and self-assembly. There has recently been a renaissance in our understanding of this important class of material phenomena, bringing the science of coacervation to the forefront of polymer and colloid science, biophysics, and industrial materials design. In this review, we describe the emergence of a number of these new research directions, specifically in the context of polymer-polymer complex coacervates, which are inspired by a number of key physical and chemical insights and driven by a diverse range of experimental, theoretical, and computational approaches.
\end{abstract}

\section{Introduction}

Charged soft matter is ubiquitous in both the synthetic and natural worlds, where the presence of electrostatic interactions serves as a way to imbue systems with the ability to respond to stimuli and enrich the possibilities for self-assembly. ${ }^{1}$ In this review, we consider a class of charged materials - complex coacervates that has emerged over the past few decades as particularly versatile,,$\sqrt{23}$ being found as a common interaction motif in biology, 4 while simultaneously being widely used in the chemical industry as a functional material in personal care products and foods. $\underline{5}$. 7 The widespread relevance of coacervates has led to a recent surge of research over the past decade, which has led to new fundamental scientific concepts, next-generation functional (bio)materials, and has set the stage for a new wave of modern materials that is pushing the boundaries of polymer physics and chemistry.

In this review, we describe and contextualize these recent advances in the science and engineering of polymer-polymer complex coacervates, outlining (i) the need for this flurry of research (ii) what the soft matter community has accomplished, and (iii)

\footnotetext{
a Department of Chemical and Biomolecular Engineering, University of Illinois at Urbana-Champaign, 600 S. Mathews, Urbana, IL, USA. E-mail: cesing@illinois.edu

${ }^{b}$ Beckman Institute of Advanced Science and Technology, University of Illinois at Urbana-Champaign, 405 N. Mathews Ave., Urbana, IL, USA.

${ }^{c}$ Department of Chemical Engineering, University of Massachusetts Amherst, 686 North Pleasant Street, Amherst, MA, USA. E-mail: perrys@engin.umass.edu

${ }^{d}$ Institute for Applied Life Sciences, University of Massachusetts Amherst, 240 Thatcher Road, Amherst, MA, USA
}

some of the exciting directions enabled by this research.

\subsection{What are Complex Coacervates?}

Complex coacervation occurs when two oppositely-charged molecular species undergo an associative phase separation (Figure 1), usually into a dense phase (the coacervate) and a dilute phase (the supernatant). ${ }^{2|8| 9}$ The earliest reports of this process go back almost a century, $\frac{8] 10}{10}$ with observations of liquid-liquid phase separation of biomacromolecules. The species involved in the coacervation process can include combinations of any number of charged molecules: colloids, proteins, surfactants, or polymers. 211,16 In particular, it is the charged attractions that distinguish complex coacervation from other forms of liquid-liquid phase separation, which may occur due to any number of other intermolecular interactions. $\frac{1718}{17}$ We thus restrict the scope of this review to polymeric systems that undergo phase separation primarily due to electrostatic attractions. While this definition of complex coacervation is fairly straightforward, there are an increasing number of instances where a variety of intermolecular interactions may lead to phase separation, $, 4 \mid 18,22$ meaning that complex coacervation might be only one of many possible descriptors for the system, and potentially an inadequate one at that.

\subsection{The Classical Theory of Coacervation}

The earliest theory of complex coacervation, known as the VoornOverbeek theory, ${ }^{9[23}$ established the prevailing conceptual understanding of the phenomenon; here, the translational entropy of 
the charged species competes with the electrostatic attraction between the same species. The Voorn-Overbeek model was specifically for two oppositely-charged polyelectrolytes - a polycation and a polyanion - and combined the Flory-Huggins theory of mixing for polymer solutions ${ }^{17}$ with the Debye-Hückel theory of dilute electrolytes. $24 \mid 25$ This results in the following expression for the mixing free energy $F_{V O}: \underline{923}$

$$
\frac{F_{V O}}{V k_{B} T}=\sum_{i} \frac{\phi_{i}}{N_{i}} \ln \phi_{i}-\alpha\left[\sum_{i} \sigma_{i} \phi_{i}\right]^{3 / 2}+\frac{1}{2} \sum_{i j} \chi_{i j} \phi_{i} \phi_{j}
$$

The first term on the right-hand side is the mixing entropy for each species $i$ (including polyelectrolytes, small-molecule salt ions, and solvent) with volume fraction $\phi_{i}$ and degree of polymerization $N_{i}$ that drives the system towards miscibility. The second term is a Debye-Hückel free energy that is the correlationinduced attraction between oppositely-charged electrolytes. 24,25 Here, the proportionality between the number density and volume fraction of charges is given by the factor $\sigma_{i}$. The strength of the electrostatic energy is given by the quantity $\alpha=\lambda_{B} / 2 a$; $a$ is the radius of the charged of species and $\lambda_{B}=e^{2} / 4 \pi \varepsilon k_{B} T$ is the Bjerrum length, which is distance over which the electrostatic energy is larger than the thermal energy $k_{B} T$. This contribution thus formally considers the attraction between a small, molecular charge and the average distribution of the surrounding oppositely-charged species. The original theory only considered these first two terms, however the third term in Equation 1 is often included, 26 which captures the short-range interactions included in the Flory $\chi$-parameter between each pair of species $i$ and $j \cdot \underline{17}$

This model makes predictions for the phase behavior of polyelectrolyte complex coacervation, with phase separation being observed at low salt and polymer concentrations. ${ }^{923}$ In its original manifestation, 9223 and in all but a few subsequent studies, 29,32 a major simplification is made that the polyanion and polycation species are symmetric, and can thus be considered a single component. The same simplification is also made for the anion and cation in the added salt, resulting in an effective 3-component system (polyelectrolytes, salt ions, water). Figure 2 shows the predicted phase diagram, which is analogous to the standard solventpolymer phase diagram from Flory-Huggins, 17 except the ordinate axis plots the salt concentration rather than the temperature. This correspondence is physically appealing, in that it highlights the role of added salt as a way to weaken the driving force for phase separation in the same way that temperature does for standard $\chi$-driven phase separation.

A key difference between the predictions of Voorn-Overbeek and Flory-Huggins is that, while in the latter the two phases are in thermal equilibrium, the former has two phases in chemical equilibrium. This leads to a subtle difference in the phase diagram, with the two coexisting phases in Flory-Huggins being at the same temperature (so that tie-lines denoting this coexistence are horizontal), 17 while the two coexisting phases in Voorn-Overbeek are at different concentrations. This leads to tie-lines connecting coexisting points in the phase diagram being non-horizontal. $39,23,33,36$ Indeed, Voorn-Overbeek predicts
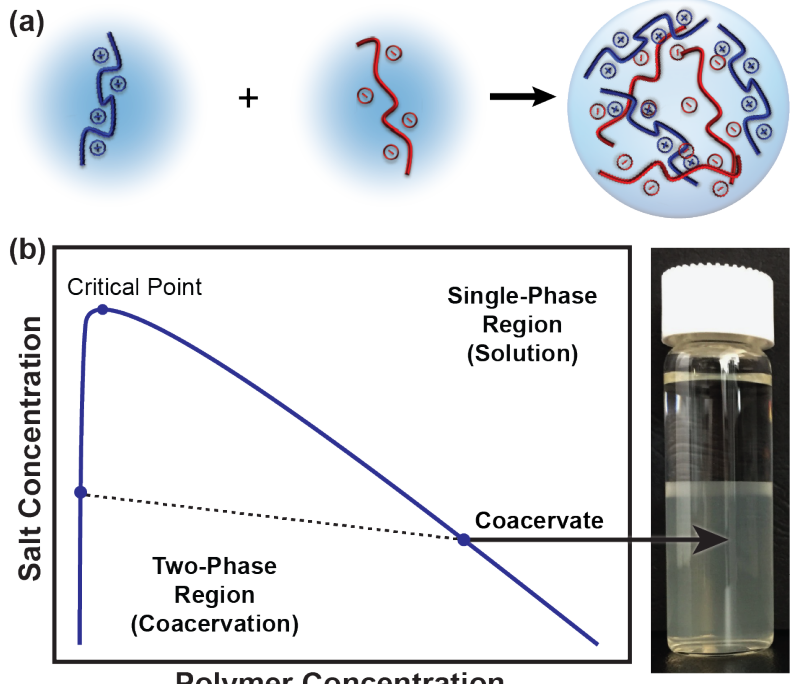

Polymer Concentration

Fig. 1 Coacervate Formation and Phase Behavior. (a) Complex coacervation occurs when two oppositely-charged macromolecules are mixed and form a dense, macroscopic phase of both species. (b) Schematic illustrating the features of a typical salt versus polymer concentration coacervate phase diagram. Coacervation occurs in the two-phase region, where there is a coexistence between the polymer-dense coacervate phase and the polymer-dilute supernatant phase. This process is shown on the right, with macroscopic phase separation leading to the coacervate phase on the bottom and the supernatant phase on the top. The line demarcating the two-phase and one-phase region is known as the binodal, which has a critical salt concentration above which the system is always miscible. The photograph shows a complex coacervate sample formed from poly(4-styrenesulfonic acid, sodium salt) (PSS) and poly(diallyldimethylammonium chloride) (PDADMAC) and 1.6 M KBr.

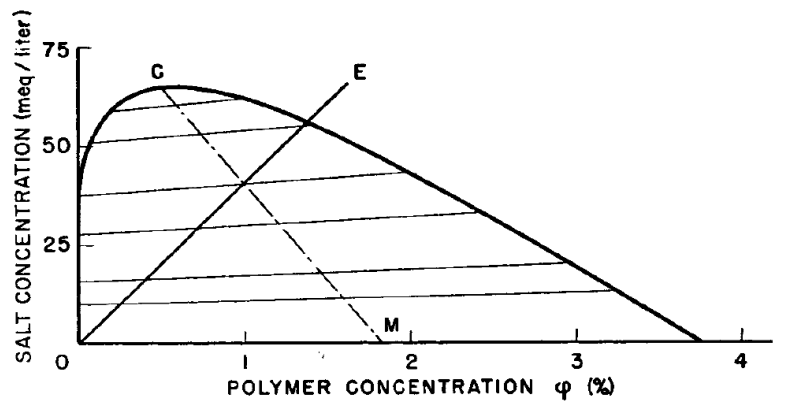

Fig. 2 Voorn Overbeek. Original salt concentration vs. polymer concentration phase diagram for complex coacervation predicted by Overbeek and Voorn. 9 Point $C$ denotes the critical point, with the line to $M$ indicating the center of the tie lines. The line from the origin to $E$ indicates conditions where the salt concentration is entirely composed of the polyelectrolyte counterions. Figure adapted from Ref. 9 , Overbeek and Voorn, J. Cell. Comp. Physiol., 1957, 49, 7-26, with permission from John Wiley and Sons. 
a positive slope for these tie lines (Figure 2);923 because the driving force for phase separation is the favorable electrostatic attraction between the positive and negative species, the small molecule salt ions preferentially partitions into the charge-dense coacervate phase.

\subsection{Experimental Measurement of Coacervation}

The Voorn-Overbeek prediction has, until recently, been the primary theory used to understand key experimental results. $923 \mid 27 / 37+39$ This is in part due to its simplicity, and the reliance on only a few key fitting parameters to match to experimental data: the charge density that sets the stoichiometric amount of charge per monomer, a dimensionless Bjerrum length that captures the strength of electrostatic interactions, and a Flory- $\chi$ parameter between the polymer and solvent species. ${ }^{27}$ Indeed, the phenomenology of coacervate phase behavior is largely captured by Voorn-Overbeek, in that phase separation is observed at low salt concentrations and leads to miscibility at high salt concentrations. 27/34/3540 A number of observables are commonly used as an indicator of phase behavior, though the primary method is to use turbidity measurements that capture the light scattering of small coacervate droplets. 3241,46 Turbid mixtures correspond to phase separation. However, other methods such as UV-Vis, $\frac{34 / 47 / 48}{3}$ or fluorescence spectroscopy, ${ }^{27 / 40 / 49}$ ionic conductivity, $\frac{34|50| 51}{3}$ thermogravimetric analysis (TGA), 35151 NMR spectroscopy, ${ }^{[50}$ radiolabelling,, 52 and quartz crystal microbalance $(\mathrm{QCM})^{53}$ have been used to measure concentrations of polymers and/or salt. Surface properties such as the interfacial tension between the surfactant and coacervate phase have been measured using atomic force microscopy 3854 and surface forces apparatus. ${ }^{55[56}$ The structure and dynamics of bulk coacervates have been characterized using rheology $37 / 47[53[57,65]$ along with X-ray and neutron scattering. $62[66 \mid 67$ Finally, the thermodynamics of bulk coacervation has been characterized using isothermal titration calorimetry (ITC). 43[50|51/68,72

Despite the wealth of reports describing and/or using coacervation over the decades, only recently has the coacervate phase diagram been mapped in a systematic way. A key paper by Spruijt, et al. ${ }^{27}$ in 2010 provided a phase diagram describing the concentration of oppositely-charged poly(acrylic acid) (PAA) and poly(N,Ndimethylaminoethyl methacrylate) (PDMAEMA) in both the coacervate and supernatant phase. These concentrations were measured via fluorescence spectroscopy using a fluorescein-labeled PAA, and assuming that an equal concentration of PDMAEMA was present. The authors explored the phase behavior for complex coacervates formed from polymers with a range of different degrees of polymerization for both the PAA and PDMAEMA, assuming that the concentration of salt was equal between the two phases (Figure $3 \mathrm{a}$ ). In this field-catalyzing effort, Voorn-Overbeek was fit to this series of experimental phase behavior data, systematically showing how parameters were chosen to match all degrees of polymerization simultaneously. 27

The success of this matching between experiment and theory in this paper spurred a renewed effort to understand these materials, along with a number of other experimental efforts emerg- ing from an interest in coacervate applications such as drug delivery 73,78 and underwater adhesives. $54 \mid 56 / 79,81$ The sense that Voorn-Overbeek is an apt (yet simple) model hinted at the possibilities of coacervation as a powerful motif in molecular design and self-assembly. While the latter part of this premise has become more apparent with time, $73,82,86$ Voorn-Overbeek has now been shown to provide a poor physical picture of coacervation. ${ }^{33|34| 87}$ Rather, the ways in which Voorn-Overbeek fails as a model have led to many of the most interesting and promising developments in complex coacervation, and have highlighted the ways in which these materials are amenable to molecular-level design of their phase behavior, dynamics, structure, and other material properties.

\subsection{The Historic Need for a More Refined Picture}

\subsubsection{The Theoretical Limitations of Voorn-Overbeek}

Despite the ability to match Voorn-Overbeek theory to experimental data, 27 from a theory perspective this agreement is surprising. A number of the assumptions built into the theory do not apply in the regime where coacervation occurs; namely, at the high charge densities and concentrations typical of these materials (i.e., synthetic systems typically have a concentration of charged species of the order of $1 \mathrm{M}) .27 / 34 \mid 35[40,43 \mid 45$

1. Debye-Hückel theory is a limiting law for low salt concentrations. Debye-Hückel solves the linearized PoissonBoltzmann equation around a single salt ion,24|25 capturing the mean-field environment around this charged particle. The thermodynamic result is thus a first-order fluctuation effect, which is known to only work in dilute, weak electrolytes (monovalent salts at concentrations below 5 $\mathrm{mM}) .25$ This is ca. 2-3 orders of magnitude lower than typical concentrations for coacervates, which are often at 0.5-3 M. 27/34/35/40,43/45

2. Polymer charges are treated as unconnected electrolyte particles in Voorn-Overbeek. There is no distinction made, in the classical Voorn-Overbeek model, between the salt ions and the charges along the polymer. This is despite the real, physical system, where polymer charges are connected with an (often) high linear charge density.

3. Voorn-Overbeek treats salts, polymers as point particles. At concentrations $>1 \mathrm{M}$, the finite size of the charged species is non-negligible. Experimental measurements have shown that bulk water only takes up somewhere around 70-90\% of the coacervate volume. ${ }^{27}$ This means that the remaining combination of salt ions, polymer, etc. comprise more than $10 \%$ of the system volume. Furthermore, the hydrated size of these species would be expected to be even larger. For instance, calorimetry measurements have suggested that the "non-freezing water" content of protein-based coacervates could be as high as $15 \% \mathrm{w} / \mathrm{w}$.

4. The solvent is assumed to be a continuum. In VoornOverbeek, water solvent is included simply via the relative 
(a)

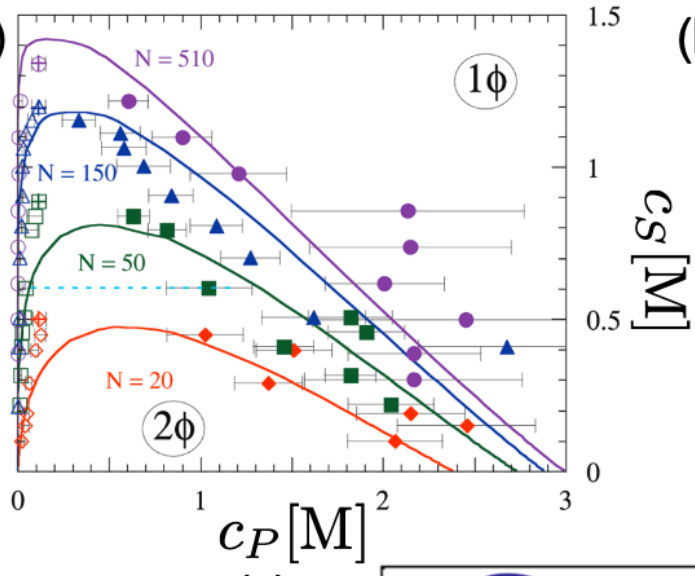

(c)

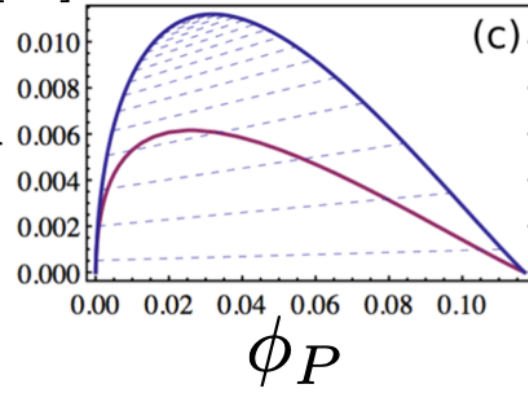

(b)

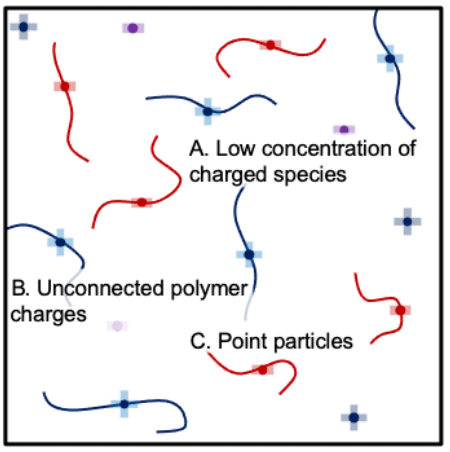

Voorn-Overbeek

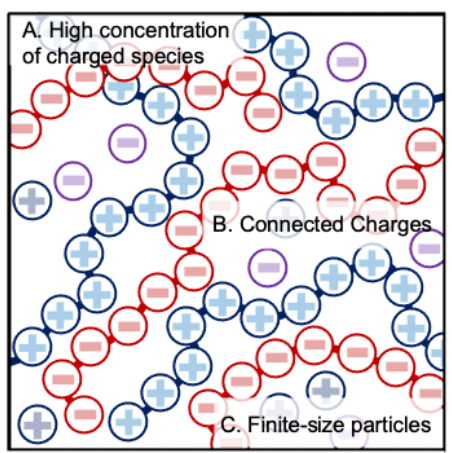

Actual

(d)

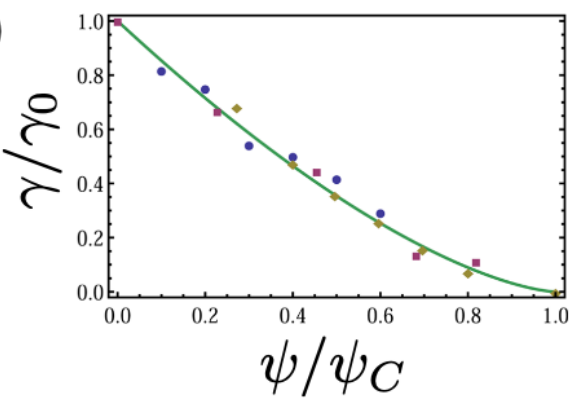

Fig. 3 Voorn Overbeek. (a) Experimental phase diagram from Spruijt et al.,27 for PAA/PDMAEMA of different molecular weights. The average degree of polymerization is indicated on the graph. Lines represent fits to Voorn-Overbeek theory, ${ }^{923} 27$ demonstrating that matching is possible with judicious choice of fit parameters. Reprinted with permission from Ref. ${ }^{[27}$ Spruijt et al., Macromolecules, 2010, 43, 6476-6484. Copyright 2010 American Chemical Society. (b) Schematic illustrating key assumptions associated with Voorn-Overbeek theory, with the left providing a schematic glimpse of what Voorn-Overbeek predicts, while the right contrasts some of the features that Voorn-Overbeek neglects. A: The Debye-Hückel term assumes a low concentration $(<5 \mathrm{mM})$ of charged species, yet experimental coacervate phases are typically $>0.5 \mathrm{M}$. B: Voorn-Overbeek does not distinguish between unconnected salt species and polymer species, except in the translational entropy. $C$ : The Voorn-Overbeek theory assumes point particles, not accounting for the finite size of both salt and polymer species. (c) Voorn-Overbeek prediction, in the salt concentration ( $\psi$ ) versus polymer concentration $(\phi)$ plane. The purple curve illustrates the prediction if the salt concentration $\psi$ is the same between both phases, but the blue curve is the prediction from Voorn-Overbeek if the salt can partition unevenly between the phases. Here, the positively-sloped tie-lines indicate that salt preferentially partitions to the coacervate phase. $\frac{39}{(d)}$ Voorn-Overbeek can match experimental predictions for the scaling of interfacial tension $\gamma$ with salt concentration $\psi$. Theory is the green line, points indicate experimental data from the literature. 383988 (c,d) Reprinted with permission from Ref. 39 Qin et al., ACS Macro Letters, 2014, 3(6), 565-568. Copyright 2014 American Chemical Society. 
dielectric constant in the Bjerrum length $\lambda_{B}$. This approximation, along with the typical inclusion of the hydration shell in the ion radius, is a way of coarse-graining the response of molecular water dipoles to charges. However, it is understood that solvent structure plays a quantitative role in coacervate thermodynamics, both in terms of Hofmeisterlike specific ion effects ${ }^{45}$ and with the 'effective' dielectric constant presumably lower in the polymer-dense coacervate phase. 90

All of these limitations have been appreciated since the original development of Voorn-Overbeek theory, and most theoretical efforts since have focused on moving beyond this simplified picture. 33 Still, the fact that Voorn-Overbeek gets many aspects of the phenomenology qualitatively correct has led to its continued use; 27/28 39|40|97 there are only a few key experimental observations in simple homopolyelectrolyte coacervates that highlight the need to move beyond this simple picture. 35]40/47,98

\subsection{2 'Beyond Voorn-Overbeek' Observations in Coacerva- tion}

Perhaps the most persistent yet subtle challenge of coacervation is that it is indeed possible to fit simple phase-behavior representations to most candidate theories, including Voorn-Overbeek. This is best exemplified by the work of Spruijt et al., which used VoornOverbeek theory combined with a Flory- $\chi$ parameter to parameterize a number of experimental phase diagrams (Figure 3 a);27 successful matching of Voorn-Overbeek theory to experimental interfacial tension measurements (Figure 3d) likewise show how challenging it is to determine that a theory is physically meaningful. $\frac{39}{}$ In an effort to refine the theoretical picture of coacervation, it is thus important to highlight the situations in which VoornOverbeek breaks down experimentally, which must be explained by successful theories:

1. Salt partitioning. Inspired by simulation and theory ef-

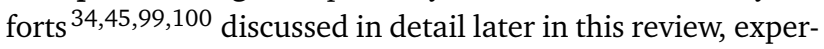
iments have recently found that most coacervation-driven phase separations exhibit a higher salt concentration in the supernatant phase than the coacervate phase.34/35/47/101 This is opposite of what Voorn-Overbeek predicts. 923

2. Entropy versus enthalpy of coacervation. Thermodynamic characterization of coacervation has demonstrated that mixing of the two oppositely-charged polymers is highly entropic, while typically having only minor enthalpic contributions to the overall coacervation process. $43|51| 69,71$ This contrasts with Voorn-Overbeek theory, which considers coacervation to be an enthalpic process driven by the increased electrostatic attraction between the charged components. 9223

3. Molecular or physical justification for fitting parameters. Previous efforts to match Voorn-Overbeek to experiment have been transparent about the fitting parameters used, 27 however it is unclear that the parameters themselves are physically reasonable for the polymers considered. While quantitative, a priori prediction of model parameters is not generally a reasonable expectation for simplified theoretical models. Knowledge of the underlying polymer chemistry should be qualitatively consistent with the interpretation of the parameters used in the model.

These experimental observations have, in part, spurred the further development of coacervate theory and thermodynamic study. However, as the candidate theories have proliferated, there has been a concomitant effort from the experimental side to develop more ways to test and probe the regimes of validity for coacervation models, and to decide for a given system the most appropriate physical explanations of coacervation.

\subsection{The Coacervation Proto-models}

The limitations of Voorn-Overbeek theory have long been wellunderstood, and progress beyond this historical framework has stemmed from a few different models that provide ways to - at least partially — address the aforementioned limitations. 33 We consider three foundational proto-models that have informed the modern approach to this problem; (i) polymer field theory, (ii) scaling theory, and (iii) counterion release models.

\subsubsection{Polymer Field Theory}

Polymer field theory has the closest connection to the original Voorn-Overbeek model, which is a simple manifestation of an underlying field theory approach (Figure 4 a). ${ }^{112}$ The field theoretic model seeks to solve a partition function $\mathscr{Z}: 113[114$

$$
\mathscr{Z}=\mathscr{Z}_{0} \int \prod_{A}\left[\mathscr{D} \phi_{A} \mathscr{D} \omega_{A}\right] \mathscr{D} \psi \mathscr{D} \eta \exp \left(-\mathscr{H}\left[\left\{\phi_{A}\right\},\left\{\omega_{A}\right\}, \psi, \eta\right]\right)
$$

This partition function accounts for the thermodynamic effect of all possible molecular configurations. ${ }^{25}$ In this system, $\mathscr{Z}$ is evaluated by sampling all possible values of density fields $\phi_{A}(\mathbf{r})$ and chemical potential fields $\omega_{A}(\mathbf{r})$ for all species $A$; here, $\mathscr{D}$ denotes integration of these densities/potentials at all possible locations r. This integral also considers all possible electrostatic potential $\psi(\mathbf{r})$ and constraining fields $\eta(\mathbf{r})$, where the latter sets the sum of volume fractions to $\sum_{i} \phi_{i}=1$. The Hamiltonian $\mathscr{H}$ is a functional of these fields, quantifying their relative importance via the Boltzmann factor $\exp (-\mathscr{H})$ that gives significant weight to fields with low values of the free energy. This Hamiltonian is written to account for the electrostatic potential $\psi$ and (often) a $\chi_{i j}$-parameter that includes information related to the short-range interactions between species $i$ and $j: 113[114$

$$
\begin{aligned}
\mathscr{H}\left[\left\{\phi_{A}\right\},\left\{\omega_{A}\right\}, \psi, \eta\right]=-\sum_{A} n_{A} \ln Q_{A}\left[\omega_{A}, \eta, \psi\right]+ \\
+\rho_{0} \int d \mathbf{r}\left[\frac{1}{2} \sum_{i, j} \chi_{i j} \phi_{i}(\mathbf{r}) \phi_{j}(\mathbf{r})+\eta(\mathbf{r})\left(\sum_{i} \phi_{i}(\mathbf{r})-1\right)-\right. \\
\left.-\sum_{i} \omega_{i}(\mathbf{r}) \phi_{i}(\mathbf{r})+\sum_{i} q_{i} \phi_{i}(\mathbf{r}) \psi(\mathbf{r})-\frac{|\nabla \psi(\mathbf{r})|^{2}}{8 \pi \lambda_{B}}\right]
\end{aligned}
$$

Here, the Bjerrum length $\lambda_{B}=e^{2} /\left(8 \pi \varepsilon_{0} \varepsilon_{r} k_{B} T\right)$ represents the length scale over which the electrostatic energy two unit charges $e$ in a medium with relative dielectric constant $\varepsilon_{r}$ ( $\varepsilon_{0}$ is the vac- 


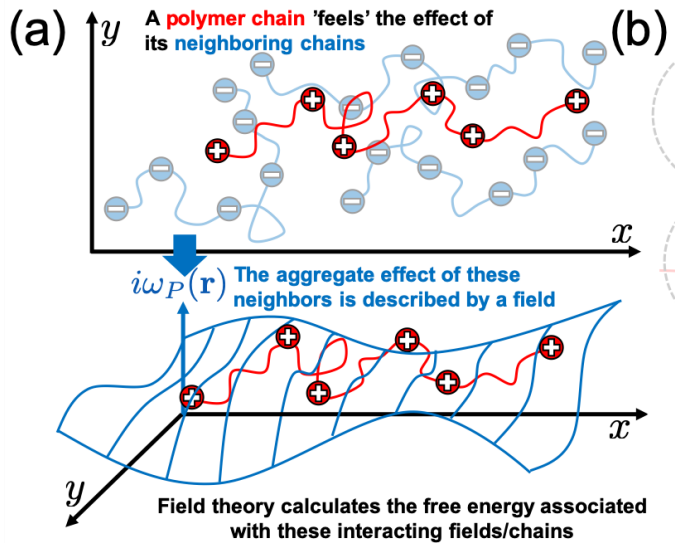

with these interacting fields/chains (b)

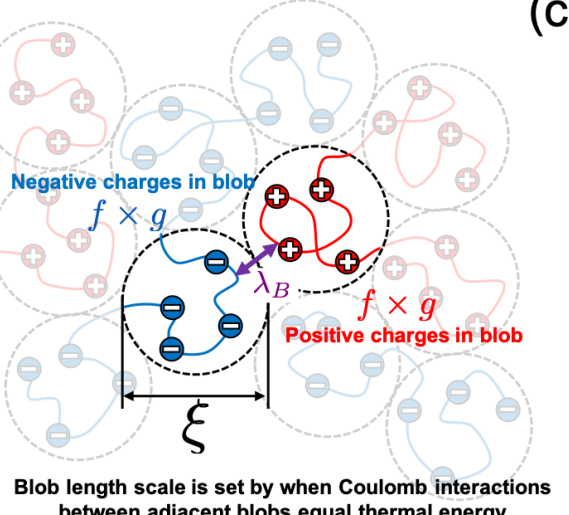

(c)

between adjacent blobs equal thermal energy

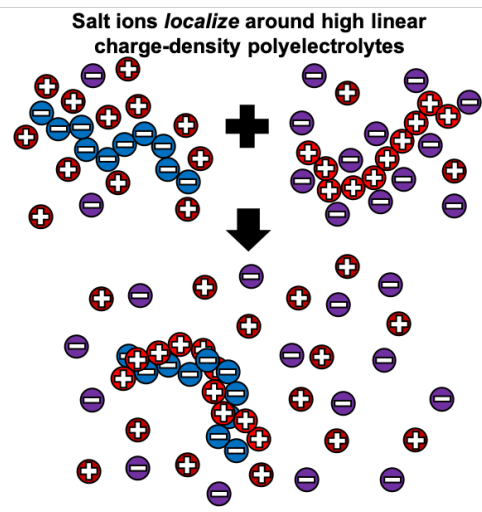

Complexation enables previously-localized salt ions to delocalize, or 'release', gaining translational entropy

Fig. 4 Coacervation Proto-Models. (a) Polymer field theory describes the aggregate effect of neighboring particles by spatially varying 'fields' describing their local density and excess chemical potential (the latter denoted here as $i \omega_{P}(\mathbf{r})$ ). A variety of modeling choices can be made, depending on (i) the extent that different possible fields accounted for in sampling the Hamiltonian in Eq. 3 and (ii) the way that the connectivity and excluded volume of the charged polyelectrolytes are modeled. $\frac{102}{1}$ The most sophisticated versions of this type of theory use complex Langevin approaches that stochastically sample the entire set of field configurations. $102-106$ (b) Scaling, or 'blob' theory, uses the comparison between thermal and electrostatic energy to determine key length scales. The length scale of each 'blob' consists of $f \times g$ charges (i.e., the number of monomers times the charged monomer fraction), where their arrangement on a Gaussian chain is enough such that their electrostatic interactions with neighboring blobs overcome $k_{B} T .107 / 108$ (c) In counterion condensation and release, ${ }^{109}$ salt ions localize around isolated high linear charge-density polyelectrolytes at the cost of their translational entropy. 1101111 This translational entropy can be regained if two oppositely-charge polyelectrolytes localize around each other, such that they no longer need to localize small molecule ions.

uum permittivity) is the same as the thermal energy $k_{B} T$. The charge on each species is given by $q_{i}$, the overall number density of the system is given by $\rho_{0}$, and $n_{A}$ is the total number of species $A$. Finally, the functional $Q_{A}$ is the single-chain partition function, which bookkeeps the Boltzmann weights for all possible polymer conformations in a field of their neighbors. The terms of this equation thus correspond to (i) the set of possible single-chain conformations in the presence of the fields $\omega_{A}, \eta$, and $\psi$, (ii) the short-range interactions between all species at all locations $\mathbf{r}$ as determined by $\chi$, (iii) a Lagrange multiplier to enforce incompressibility, (iv) a Legendre transform in order to treat each location $\mathbf{r}$ as an open system in equilibrium with neighboring grid points, (v) the effect of the electrostatic field on the species charges, and finally (vi) the energy of the electrostatic field itself.

Analytical evaluation of this (or related) models uses a hierarchy of approximations of this otherwise-intractable partition sum. ${ }^{99100|112| 115] 116 \mid}$ These approximations are based on expanding the Hamiltonian around a single set of 'mean' fields, which for bulk coacervation are characterized by concentrations of salt and polymer species that are homogeneous throughout space. ${ }^{102}$ The simplest level in this hierarchy replaces the partition sum over all possible states with only this homogeneous state, assuming that it dominates the partition sum.102 This mean-field approximation is known to be insufficient for all but the weakest-interacting charged systems, because the square gradient term in Eq. 3 disappears for a homogeneous state, resulting in the absence of electrostatic energy. ${ }^{113}$ This term only serves to maintain electroneutrality in homogeneous states, and becomes non-zero only in inhomogeneous systems. $\frac{113 \mid 114}{1}$ This is a problem for coacervation, where the homogeneous coacervate phase occurs due to electrostatic interactions that - in this representation - effectively disappear. The mean-field theory thus cannot predict coacervation.
Further expanding the Hamiltonian to quadratic order is known as the Random Phase Approximation (RPA),117 and is widely used because of the ability to solve the resulting Gaussian integrals in the partition function. The simplest version of this treatment leads to the Voorn-Overbeek theory, ${ }^{923] 112]}$ which results when there is no chain connectivity included in the Hamiltonian. Modifications were first introduced by Borue and Erukhimovich, 115 and subsequently Castelnovo and Joanny, ${ }^{116}$ who used the one-loop expansion of the RPA to predict the effects of salt and $\chi$ on coacervation. Generalizations of these ideas are frequent in the literature; Olvera de la Cruz included short-range correlations via a diagrammatic representation of ion pairs as 'reversible cross-links' and also included a high-q modification to approximate the effect of finite excluded volume. ${ }^{99 \mid 100}$ More recently, Qin and de Pablo demonstrated how the inclusion of chain connectivity - in particular the fractal dimension of the connectivity (e.g., rods versus coils versus branched polymers) plays a significant role on coacervation. 112

\subsubsection{Scaling Theory}

Scaling theory uses so-called 'blob' arguments, that establish relevant length scales in polymer physics via comparison between thermal and other energetic (in this case, electrostatic) aspects of the system (Figure $4 \mathrm{~b}$ ) ${ }^{1171118}$ Scaling arguments for coacervation rely on the competition between the electrostatic attraction between oppositely-charged blobs and the local conformational fluctuations within each blob. 107/108|119!121 The original scaling picture of coacervation considered the self-complexation of diblock polyampholytes,, $107 / 108$ containing both a positively and negatively-charged block. This led to an equivalence between the 
thermal energy and the energy of adjacent 'blobs' of size $\xi$ :

$$
\left|\frac{\lambda_{B} f^{2} g^{2}}{\xi}\right| \approx 1
$$

Here, the strength of the electrostatic interaction over the 'blob' length $\lambda_{B} / \xi$ is multiplied by the number of charges on each blob (two contributions of $f g$, where $f$ is the charge fraction and $g$ is the number of monomers in each blob). This resulting 'scrambled egg' model correspondingly predicts the concentration of a coacervate in the salt-free case,, 107 and also the interfacial tension of a coacervate. $\frac{108}{}$ Recently, this model has become the basis for further study into coacervation phenomena; for example, polyelectrolytes with vastly different linear charge densities have been studied,, 119 showing analogies with simple polyelectrolytes. ${ }^{122}$ This model has also formed the basis for scaling theories of coacervate-core micelles, and the transitions between different micelle geometries. $\underline{123}$

\subsubsection{Counterion Release}

Counterion release stems from an observation in the early polyelectrolyte literature that, for high charge density polymers, there was a divergence in the electrostatic potential that was only solvable if the 'effective' charge density of the chain was renormalized to be below a critical value. ${ }^{110}$ Physically, this 'Manning condensation' effect suggests that oppositely-charged counterions are strongly localized near to a polyelectrolyte chain, while at larger distances the polyelectrolyte appears to have a charge density related to the inverse Bjerrum length $1 / \lambda_{B}$. This counterion localization comes at the cost of the translational entropy of the counterions. A number of theoretical and computational efforts have considered this charge localization phenomena.110|111/124/130

Counterion release occurs during complexation between oppositely-charged polyelectrolytes, which can effectively 'condense' on each other (Figure 45). This removes the driving force for the localization of counterions, which regain their translational entropy.29109 This effect is often invoked during the complexation of two individual polyelectrolytes, $\frac{109}{134}$ and is directly apparent from molecular simulation.131.134 Despite the widespread use of this concept to describe complexation of pair complexes, 29109 only recently has charge localization been regularly invoked in understanding bulk coacervation. 31971135

\subsection{Moving Beyond the Proto-models}

Each of the models considered above provides some insight into the nature of coacervation; however, the limitations and connection between the models is often unclear. For example, length scales that emerge from RPA approaches often agree with those derived by scaling approaches.1121121 Also, the counterion release model specifically considers high charge-density polymers, 110 while fluctuation-induced attractions in scaling and field theoretic approaches are more accurate in the opposite limit. ${ }^{33|112| 116[119}$ Ultimately, progress in understanding the physics of coacervation is tied to specific experimental challenges and questions, and in recent work this has informed the choice in theoretical or computational model.

\subsection{Key Experimental Challenges}

The development of a detailed and predictive understanding of complex coacervation has historically been limited due to the poorly defined chemical and physical properties of many of the naturally-derived polymers that dominated the literature.51612]13]58 Recent advances in controlled polymerization have enabled the synthesis of a diverse palette of chemically and physically well-defined polyelectrolytes that can be used to test specific hypotheses. For instance, synthetic polypeptides have been used extensively as a model polymer system where different side chain functionalities can be introduced along the same backbone. $35[43 / 45[55 / 75 / 77 / 136$ Additionally, solid-phase synthesis enables precise control over chemical sequence, $, 70 \mid 137,[143$ and can be combined with methods for controlled polymerization to allow for the preparation of well-controlled comb polymer architectures. ${ }^{46 / 144}$ Controlled polymerization also has allowed for the synthesis of random copolymers to facilitate the introduction of multiple functionalities. ${ }^{64}$ Lastly, the expanding breadth of synthetic approaches for preparing charged polymers has allowed for the design of elegant experiments to test specific molecular features, such as hydrophobicity ${ }^{53}$ or polarity. 40

Another key challenge for both experiments and for theoretical descriptions is the need to understand how the specific chemical identity of both the polymers and salt ions affect coacervation. Here, the difficulty is the need to understand both the chemical details of the molecules themselves, and how those details affect their interactions with water as the solvent. ${ }^{[145]}$ For instance, changes in the physical size of atomic salts affects the hydration shell of these ions. This hydration behavior then correlates with the strength of their interactions with charged groups on the polymer.146-148 However, the chemical identity of even the neutral aspects of the polymer can further modulate the structure of surface bound water. For all of these questions, the challenge is one of trying to understand changes in the structure of water. To date, the majority of approaches rely on indirect phenomenological measurements such as changes in the phase behavior of coacervates, ${ }^{40|64| 139}$ differences in the amount of water introduced into a sample by different ions,, 53 or measurements of "non-freezing," surface-bound water. ${ }^{89}$ Experimentally, new techniques such as terahertz dielectric spectroscopy have the potential to help directly access these structural changes, 1491150 and these questions look to be key to future materials design questions even beyond coacervation.

\section{Recent developments in the science of complex coacervation}

\subsection{Phase Behavior of Polymer-Polymer Coacervation}

A main scientific focus of the past ten years of effort in the community has been to understand - and predict - phase behavior in a fashion that reflects its molecular origin. As such, the focus has been on isolating and then converting the effect of a number of molecular features into an understanding of coacervation. We highlight a few key attributes; charge connectivity, salt partitioning, polymer architecture, and polymer stiffness. 


\subsubsection{Polymer Connectivity}

The lack of connectivity between charges is immediately apparent as a limitation of the Voorn-Overbeek theory, ${ }^{|9| 23133}$ as it is an obvious aspect of the polyelectrolyte molecular structure. Indeed, the original paper by Voorn-Overbeek explicitly discusses this approximation; ${ }^{9}$ yet, it remains a major challenge to isolate its effect on coacervation. Theory has provided a number of insights into the role of connectivity, from two different perspectives; via fluctuation-induced opposite-charge attraction, or via counterion condensation and release. The current, prevailing view is that both effects can induce coacervation, 151 in the low- and highlinear charge density limits respectively, and this understanding is reinforced by evidence from a combination of theory, simulation, and experiment.

Fluctuation-induced attraction arises due to the coiled structure of the polyelectrolyte chains, where like-charges are spatially nearby due to chain connectivity. This competes with the attraction between charges on oppositely-charged polyelectrolyte chains, which drives these coils to overlap significantly to increase opposite-charge attraction (Figure $5 \mathrm{a}$ ). This is the operative driving force for coacervation when the linear charge density is low, and at some length scale chain segments are unperturbed by electrostatic repulsion or attraction (i.e., the 'electrostatic blob' in scaling theory).122126 This concept is the operating principle of most historical theories; for example, scaling theories such as the one developed by Rubinstein use the concept of both the electrostatic and concentration 'blobs. 107/108|119 In this framework, coacervation occurs when the electrotatic attraction between the adjacent blobs is of the order of thermal energy $k_{B} T .107$ 108 119/123 Here, the coiled chain structure enters as the connection between the concentration and the size of the component 'blobs', which get progressively smaller as the linear charge density increases, and correspondingly the coacervate concentration increases. This fluctuation-induced attraction is similarly the basis for theories built on RPA, such as the work by Borue and Erukhimovich, 115 Joanny and Castelnovo, 116 and Kudlay and Olvera de la Cruz. 99100

Recent extensions of both field theory and scaling ideas have expanded on the role of chain connectivity in fluctuation-induced attraction, in a number of different scenarios. Qin and de Pablo provided a unifying picture of analytical theory in the low linear charge density limit, by expressing the RPA result of the correlation free energy for molecules with arbitrary dimensionality $d$ in a general form: 112

$$
f_{\text {corr }}=\frac{\csc (3 \pi /(d+2))}{12 \pi} \frac{v}{\lambda^{3}}
$$

The value $v$ is a reference volume, and the parameter $\lambda \sim$ $\lambda_{0} \phi^{-1 /(d+2)}$ is the concentration-dependent correlation distance. This distance $\lambda$ decreases with polymer density $\phi$ due to increased charge screening, and is also related to the molecular dimensionality that sets the compactness of polymer charges (i.e., branched versus linear structures) ${ }^{112}$ Here, we note the key components of fluctuation-induced attraction; the coiled chain structure is represented by the dimensionality $d$, with an electrostatic interaction that strengthens with increasing polyelectrolyte volume fraction $\phi$.

This class of theoretical results provides analytical expressions due to the approximation that the charge species have density fields exhibiting Gaussian fluctuations. 99]100|112]116 A complementary, computational field theory approach has been used to move beyond this simplifying assumption, and simultaneously enabled the study of inhomogeneous (i.e., spatially-varying) features of coacervate systems such as interfaces and self-assembled structures. A series of papers by Fredrickson, et al. have used complex Langevin simulations to calculate the full field theory (Figure 5b) $188,103,106$ Connectivity is inherently included in the calculation of these field theories, usually as adjacent segments of a Gaussian chain that are connected via Hookean springs. $\frac{88102-106}{10}$ In most recent field theoretic implementations, charge is spread around these segment locations via a Gaussian distribution, and is combined with a soft chain-chain repulsive interaction that includes the effect of excluded volume.104 At this level of coarse-graining, the model approximates the locallyunperturbed chain segments of charge as individual entities, but the field theory fully captures how these entities themselves are connected and arranged in the coacervate. 103

Scaling theory has been used to further probe the role of chain connectivity, including recent work by Rumyantsev et al. that maps out the effect of solvent quality and salt concentration (Figure $5 \mathrm{c}, 120$ and by Rubinstein, focusing on the structure of coacervates with disparate linear charge-densities (Figure $5 \mathrm{~d}$ ). ${ }^{[19}$ The former describes two different behaviors; at low salt a single length scale is set by the electrostatic blob due to the screening between oppositely-charged chains, while at high salt the screened electrostatics is smaller than the concentration blobs (Figure 5, bottom). ${ }^{120}$ Rubinstein subsequently uses scaling blob arguments to explain how a relatively high linear charge density polyanion induces the formation of a surrounding 'coat' of low linear charge density polycation. 119 This leads to two characteristic length scales, and establishes an analogy with the structure of polyelectrolytes, 126 with the low linear charge density polycation playing a similar role to the counterions in standard scaling theories of polyelectrolyte solutions. This model predicts the existence of a peak in the polyanion/polyanion scattering function, 119 which was verified by simulation and can in principle be tested by scattering experiments (Figure $5 \mathrm{~d}$, bottom) $\sqrt[6266]{6 i} \mathrm{Fi}$ nally, this theory also offers a rough prediction for where the assumption of low linear-charge density is no longer applicable, 119 which is where the electrostatic blob is roughly the same size as an individual monomer, corresponding to the high charge-density limit.

Counterion release and ion pairing arguments are generally motivated by a need to describe the high linear chargedensity limit, characterized by the significant localization of opposite charges to polyelectrolyte chains. 110|111|124|152 This has long been shown to exist in simulation, $111|129| 131$ with the complexation of pairs of oppositely-charged chains corresponding directly to the release of their counterions. $\frac{109}{}$ The primary theoretical challenge has been to incorporate the concept of counterion condensation and release into a model of bulk coacervation, be- 
(a)
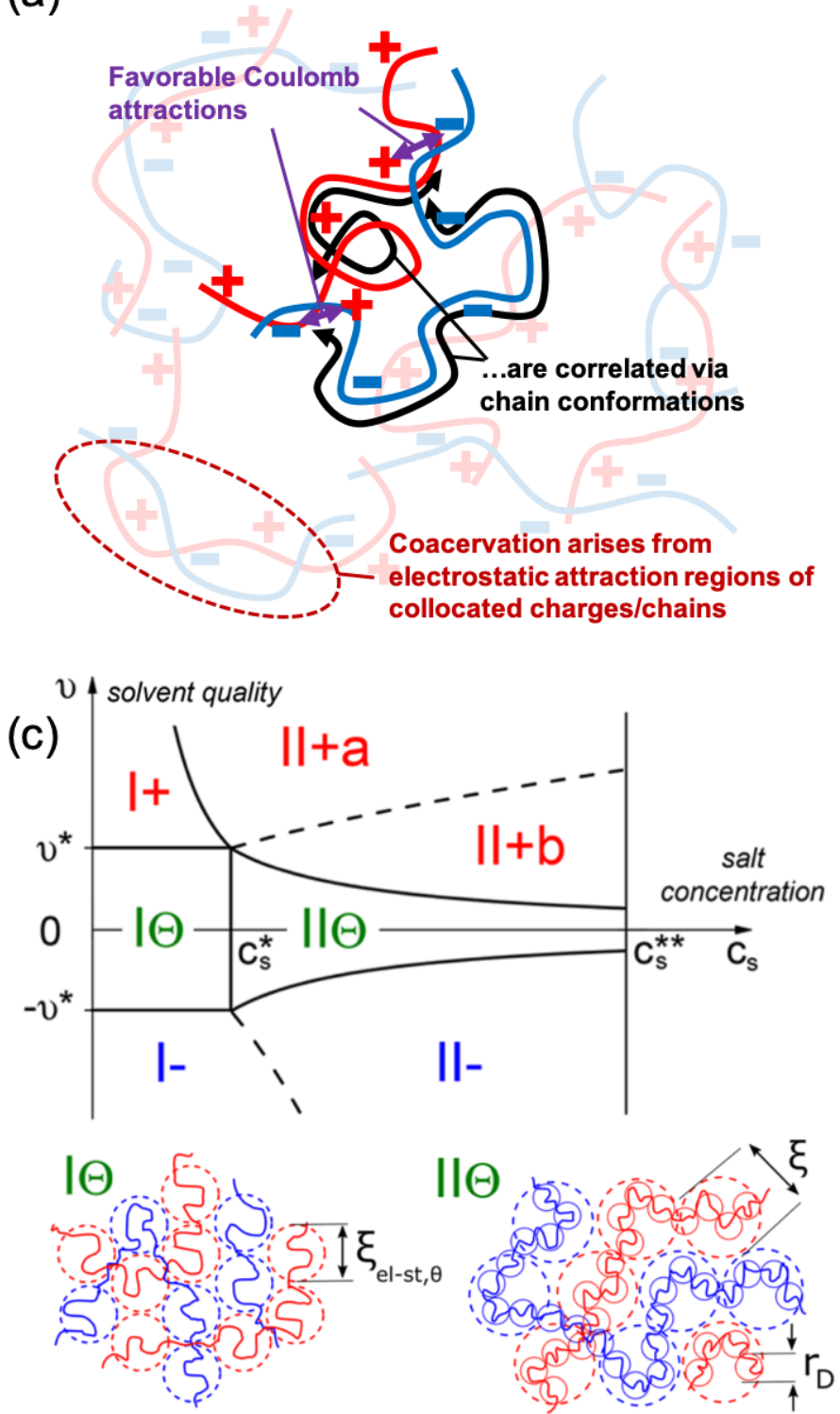

Length scale set by electrostatic blob (i.e. chain-chain screening)
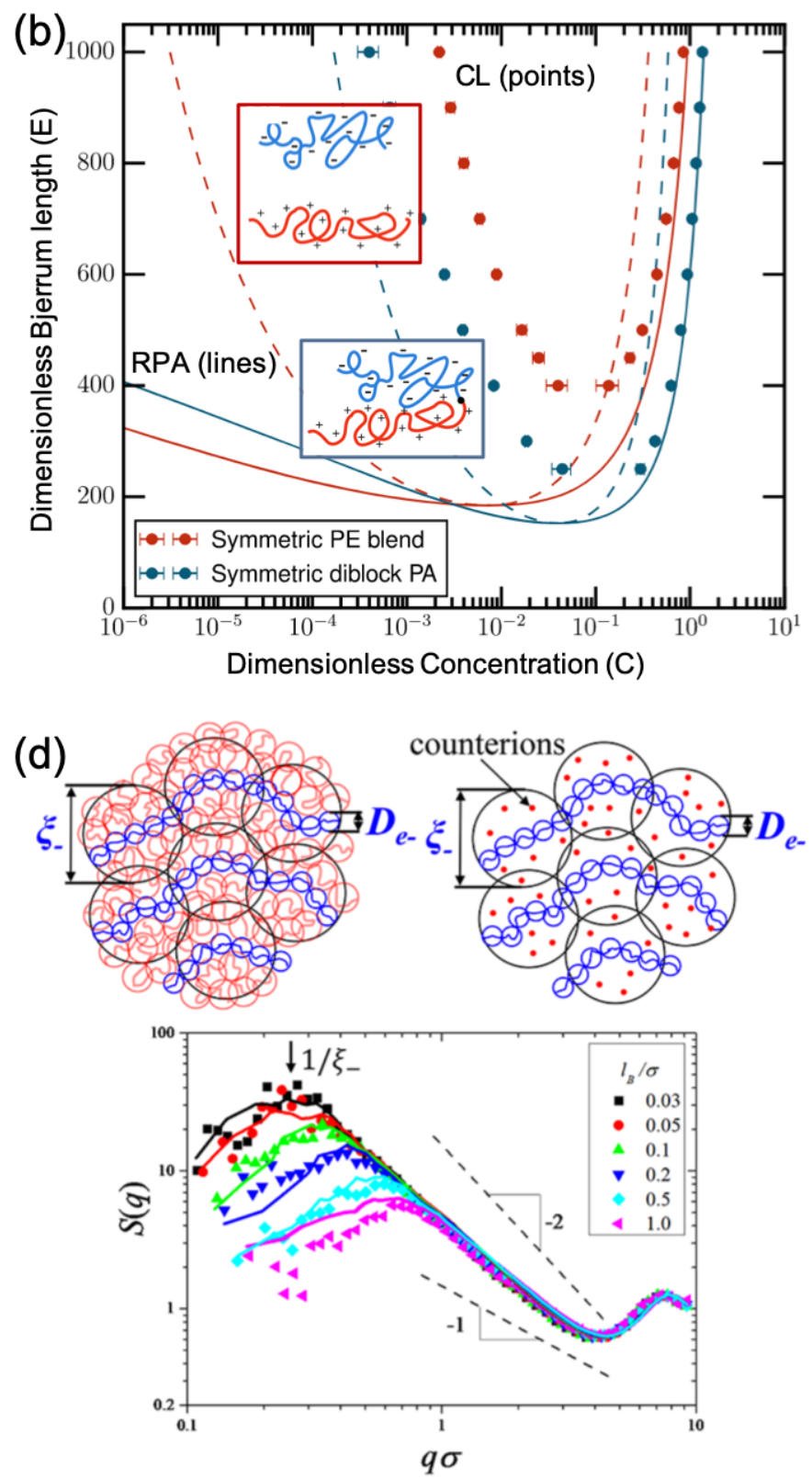

Fig. 5 Fluctuation-Driven Phase Separation. (a) Schematic illustrating the principle of fluctuation-driven coacervation. Favorable Coulomb interactions are correlated to each other via connectivity, often assuming a Gaussian or near-Gaussian chain conformation. In this way, there is a collocation of charges, enhancing their electrostatic attraction. Field theory and scaling approaches are governed by these key ingredients, with a variety of different levels of sophistication. (b) Work by Delaney and Fredrickson showcases how different levels of model sophistication can lead to different results. 104 Predictions from a full field theoretic phase diagrams (binodals) of both polyelectrolyte and polyampholyte coacervation processes are shown as points, which consider the full set of possible concentration fluctuations. Lines indicate binodal (solid) and spinodal (dashed) predictions from the Random Phase Approximation, which accounts only for Gaussian distributed fluctuations. Here, phase behavior is plotted as a function of dimensionless parameters that account for the strength of electrostatic interactions $(E)$, and the polymer concentration $(C)$. Reprinted from Ref. 104 , Delaney and Fredrickson, J. Chem. Phys., 2017, 146(22), 224902, with the permission of AIP Publishing. (c) Scaling predictions also provide fluctuation-based predictions. This phase diagram by Rumyantsev et al., delineates two regimes; 120 where salt concentration is low and the coacervate concentration is dictated by 'electrostatic blobs' $\xi_{e l-s t}$ where chains screen each other, and $I I$ where the mesh size $\xi$ is determined by the concentration and electrostatics is screened at small length scales $r_{D} .120$ Illustrative schematics shown for $\Theta$-solvent, but different regimes are observed as solvent quality becomes good/bad. Reprinted (adapted) with permission from Ref. 120 Rumyantsev, Zhulina, and Borisov, Macromolecules, 2018, 51(10), 3788-3801. Copyright 2018 American Chemical Society. (d) Scaling arguments by Rubinstein, et al. can be extended to asymmetric charge densities, leading to 'brush-like' structures analogous to polyelectrolyte solutions. 119 In these predictions, as in most scaling and field theory calculations, key length scales are determined that may be observable as peaks in the scattering function $S(q) .104 \cdot 106119$ Reprinted (adapted) with permission from Ref. ${ }^{[19]}$ Rubinstein, Liao, and Panyukov, Macromolecules, 2018, 51(23), 9572-9588. Copyright 2018 American Chemical Society. 
cause the opposite-charge localization requires the inclusion of some sort of hard-core potential between the charged species. Here, the local organization of charges - known as charge correlations - become an important aspect of describing coacervation. ${ }^{98}$ High linear charge-density is indeed the limit where field theoretic methods are known to break down;105|106|116 while it is in principle possible to perform finely-grained field theoretic simulations at this limit, $\frac{102}{10}$ such an effort would require a small grid to sufficiently capture local charge correlations (on the length scale of the ion size), and it remains numerically difficult to include hard-core interactions. $\frac{88}{}$ Two primary theoretical approaches have been taken to explore this limit; liquid state theory is capable of capturing the structure of these local charge correlations, 153 and/or ad hoc counterion condensation arguments can be used to include connectivity.

The use of liquid state theory to describe complex coacervation is a recent development, spurred by the desire to capture local charge correlations. Liquid state theory has long been a successful approach to describe the equilibrium structure of polyelectrolyte solutions $155-157$ and polyelectrolyte blends and block copolymers 158 capturing the strong counterion localization around polymers that is important for high linear-charge density coacervation. The premise of liquid state theory is to selfconsistently solve the thermodynamically-exact Ornstein-Zernike equation with an approximate closure relationship that connects pair correlation functions to an underlying interaction potential.153|163 The first attempt at using liquid state theory to describe complex coacervation, by Sing and Perry, $\underline{87}$ used a modified version of the Ornstein-Zernike equation known as the polymer reference interaction site model (PRISM): 163

$$
\hat{h}_{i j}=\hat{\omega}_{i k} \hat{c}_{k l} \hat{\omega}_{l j}+\hat{\omega}_{i k} \hat{c}_{k l} \rho_{l} \hat{h}_{l j}
$$

Here, $h_{i j}=g_{i j}-1$ is the matrix of overall pair correlation functions between species $i$ and $j$, with the hat denoting a Fouriertransformed property. The Ornstein-Zernike equation writes the correlations included as $h_{i j}$ as either the result of a direct correlation function $c_{i j}$ (first term on the right) or an indirect contribution that is a convolution of $c_{k l}$ and $h_{i j}$ (second term on the right); $\frac{153}{15}$ in PRISM, there is a further convolution with the directly connected monomers via the intramolecular correlation function $\omega_{i j} .163$ Sing initially chose this $\omega_{i j}$ term to be the known expression for a rigid rod, 164 to capture the locally stretched polyelectrolyte chain. $\frac{87}{}$ Combined with the Debye-Hückel extended mean spherical approximation (DHEMSA) closure developed by Zwanikken and Olvera de la Cruz, 165 then it is possible to write an expression for the $f_{\text {corr }}$ (or the related excess chemical potential $\mu_{\text {exc }}$ ) analogous to the correlation free energy determined in RPA-based theories. 112 What PRISM theory enables, however, is a determination of the local liquid-state charge structure for hard-core charges at high linear charge-density coacervates (Figure 6a). Indeed, the results of this theory are able to (i) have a limiting behavior equivalent to Voorn-Overbeek yet (ii) capture the effects of charge connectivity by changing the properties of the intramolecular correlation function $\omega_{i j}$. $\frac{87}{}$ Furthermore, it is possible to capture the pair correlations around the polyelec- trolyte chains to show how opposite charges localize strongly at high charge-density chains (Figure 6 a, inset). 87

Other implementations of liquid state theory by Wang et al. take a different approach, $36|166| 167$ using known expressions for excess free energy due to hard-core interactions (the BoublikMansoori-Carnahan-Starling-Leland expression), 168|169 and the excess free energy due to the Mean Spherical Approximation for unconnected charges. $\frac{36 / 153}{}$ Connectivity was incorporated perturbatively via a method developed by Wertheim, 170|171 using a pairwise assumption that approximates the long-chain connectivity in coacervates. These efforts by Wang extensively studied coacervation phase behavior (Figure 6p) beyond the initial efforts by Sing and Perry, ${ }^{87}$ for example considering the effect of nonstoichiometric charge ratios between the polyelectrolyte species. 30

Liquid state theory is a rigorous way to capture local packing in high charge-density; yet, despite advances in making the formalism more accessible, $\frac{172}{1 t}$ it remains challenging to numerically implement and is subject to approximations that emerge from the choice of closure relationship. 153 This has inspired the development of ad hoc descriptions of charge localization near high linear charge-density polyelectrolytes. Zhang and Shklovskii developed an early picture, primarily for complexation between two polymer chains, but including a prediction of bulk coacervation when oppositely-charged polyelectrolytes were near stoichiometric ratios. ${ }^{29}$ A more recent approach was used for coacervates by Salehi and Larson (Figure 6e), 97 which has been extended in collaboration with Qin (Figure 6 d), 40|154 in which there is a fraction of paired charges between the oppositely-charged chains. These approaches are analogous to the theory developed by Olvera de la Cruz for polyelectrolyte condensation with multivalent ions essentially coacervation where one species has a very small degree of polymerization - where condensed counterions can be modeled as a a fluctuating 'effective charge. ${ }^{173 \mid 174}$ In all of these efforts, connectivity is implicitly considered via the inclusion of counterion condensation, and is parameterized to match observable phase behavior. ${ }^{40 \mid 97 / 154}$ Pairing has also been considered in a model by Adhikari and Muthukumar, $135[175$ who model coacervation as the attraction between pairs of complexed polyelectrolytes. The advantage of these ad hoc models is that they do not require an explicit description of the locally condensed charges, and instead only need to be appropriately parameterized. However, in contrast to (for example) liquid state theories, these models remain limited in their ability to provide predictive insight into the behavior of the local charge correlations.

A recent development by Sing extends this picture as a way to separate the structural correlations and the electrostatic interaction energy, by mapping the three-dimensional, correlated coacervate to a one-dimensional adsorption model (Figure 6, left). 31]176/177 Each polyelectrolyte monomer is an adsorption 'site' that carries a paired ion or polyelectrolyte charge of the opposite sign, with an adsorption chemical potential that is related to the external concentration. 31 Structural correlations arise due to the ability of the polyelectrolytes run adjacent to each other, 176 leading to predictions that can match nearly quantitatively with simulation (Figure 6e, right). This simplified picture can be modified to account for a number of different connectivity effects, such 

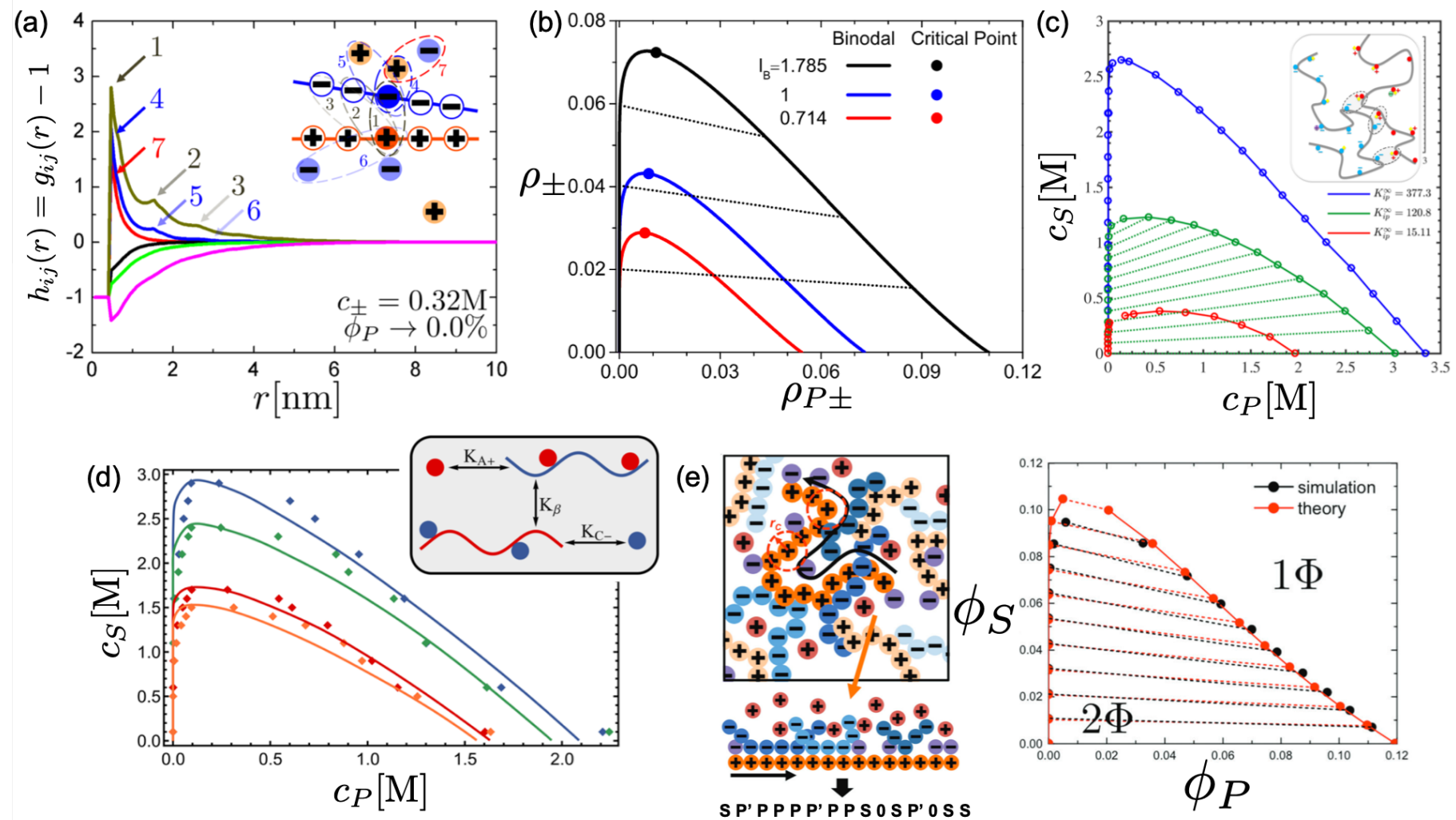

Fig. 6 Charge Localization and Counterion Condensation. (a) Pair correlations from PRISM theory, for a dilute polyelectrolyte solution. Reprinted (adapted) with permission from Ref. ${ }^{87}$ Perry and Sing, Macromolecules, 2015, 48(14), 5040-5053. Copyright 2015 American Chemical Society. Peaks in the correlation functions are attributed to neighboring charge interactions and connectivity as illustrated in the inset. Notably, these correlations are relatively short-ranged, and sensitive to molecular features. (b) Work by Zhang et al.,$\frac{30}{30}$ using a different liquid-state theory to similarly account for charge correlations in a coacervate phase diagram (salt number concentration $\rho_{ \pm}$versus polymer number concentration $\rho_{P \pm}$ ). Reprinted from

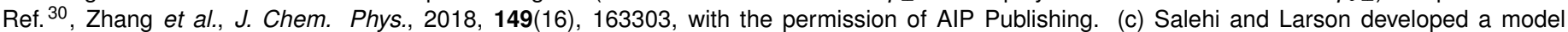
to account for this type of charge localization, in addition to $\mathrm{pH}$ effects in weak polyelectrolytes.97 They show that the extent of charge localization (modeled as a reversible reaction) strongly changes the location of the binodal. Reprinted (adapted) with permission from Ref. 97 Salehi and Larson, Macromolecules, 2016 49(24), 9706-9719. Copyright 2016 American Chemical Society. (d) Qin and Xia were able to match experimental binodal curves with an extension of the Larson model, including RPA-based electrostatics to capture long-ranged correlations and the effect of sidechain polarity. ${ }^{[54}$ Reprinted (adapted) with permission from Ref. ${ }^{40}$ Lou et al., ACS Central Science, 2019 5(3), 549-557. Copyright 2019 American Chemical Society, https://pubs.acs.org/doi/10.1021/acscentsci.8b00964. Further permissions related to the material excerpted should be directed to the ACS. (e) Lytle and Sing extended this idea to account for along-the-chain correlations (i.e., allowing for the types of local 'ladder' configurations seen in (a) where the oppositely-charged polyelectrolytes align), in a 'transfer-matrix' (TM) model. 31 The TM model closely matches simulation data. Ref. 31 , Lytle and Sing, Soft Matter, 2017, 13, 7001-7012 - Reproduced by permission of The Royal Society of Chemistry. 
as different charge spacings ${ }^{31}$ or architectures. 176

Experimental and simulation observables. Despite the wealth of theoretical efforts to model the effect of connectivity among polymer charges, it remains challenging to isolate the connectivity effect in complex coacervation. Work by Perry and coworkers provides perhaps the most direct study, 46 by varying the architecture of coacervate-forming polymers. This paper demonstrated that branches of four like-charges along a polyelectrolyte form less stable coacervates than polymers with the same number of charged monomers in the backbone.46]133[178 The key point here is that the charges are the same, but physically connected in a different way, leading to drastically different phase behavior. Satisfyingly, these experimental observations match well with both simulation and theory, ${ }^{46 / 176}$ lending credence to the less testable theoretical predictions.

In a more indirect way, simulations provide testable insight into the effect of connectivity on complexation thermodynamics. Whitmer et al., used expanded-ensemble algorithms with coarse-grained Monte Carlo simulations to show that the different complexation mechanisms exist at different linear charge densities, with high charge-density polyelectrolyte complexation being entropically-driven (i.e., counterion release) and low charge-density complexation driven by energetic interactions (i.e., fluctuation-induced attraction). $\frac{151}{151}$ This illustrates the connection between the two different modes of coacervation, and establishes a connection to thermodynamics as measurable by isothermal titration calorimetry. $\frac{43 / 72}{}$ Indeed, a number of examples of synthetic polymer complexes in the literature have been measured using calorimetry, $43|50| 51|68,70| 70,72$ mostly consistent with an entropic driving force. This consistency is likely due to the relative high linear charge-density of the polymers used in these studies, with each monomer carrying a charge. However, there remains a need for low linear charge-density polyelectrolyte systems that exhibit coacervation to test the transition between the two mechanisms for complex coacervation.

\subsubsection{Salt Partitioning}

Salt partitioning has emerged as an experimental observable that is at odds with Voorn-Overbeek theory, and provides a meaningful test for theoretical and computational models. $\frac{36}{36}$ This is usually denoted on phase diagrams as 'sloped' tie-lines in plots of salt concentration $\phi_{S}$ versus polymer concentration $\phi_{P}$ phase diagrams. 923 For example, a downward sloped tie line connects a supernatant (low- $\phi_{P}$ phase) at a higher $\phi_{S}$ than the coacervate (high- $\phi_{P}$ phase), meaning that salt prefers the supernatant phase (Figures 1p, 6p,d, 7b). The opposite is also possible, with a positive slope indicating that salt prefers the coacervate phase (Figure 2, 6. ). While it is widely understood that this salt partitioning occurs, phase diagrams do not always indicate this aspect of equilibrium. 2740

The original Voorn-Overbeek theory predicts a non-negligible salt-partitioning, in that case to the coacervate phase (Figure 2); ;9 23 this is driven by the fluctuation-induced attraction between all the charged species, including the salt species. This observation is consistent across most analogous field theory models, and thus a number of recent works have also reported positive tie lines (Figure 6e) $\frac{39|97| 105}{3}$ The earliest example of the opposite prediction is the work by Kudlay and Olvera de la Cruz, ${ }^{99 \mid 100}$ who explicitly include the effect of excluded volume via a modification to the form of the Coulomb potential (Figure 7p). This result is an ad hoc addition, mathematically chosen to be straightforwardly incorporated into the RPA formalism.99|100 This advance crucially captures the experimentally-observed phenomenology of salt-partitioning to the supernatant, $\frac{34}{34}$ however the magnitude of the effect is sensitive to the highly-approximate form of the excluded volume. $99 \mid 100$

Recent interest in coacervation has revived the discussion of salt-partitioning, initially via the work of Sing and Perry, who were able to use liquid state PRISM theory to reinforce the Olvera de la Cruz prediction. 87 Liquid state theory has the advantage of being able to directly include the effect of hardcore interactions, which can be directly observed via predictions of pair correlation functions. $87|153| 163$ In this work, salt partitions significantly to the supernatant phase, except as the model approaches the limit of small charges and unconnected charges (i.e., the Voorn-Overbeek limit). 87 This work was followed by simulation and experimental results, 34 also by Sing and Perry, confirming the earlier PRISM theory results. Experiments considered a coacervate formed from poly(4-styrenesulfonic acid, sodium salt) (PSS) and poly(diallyldimethyl ammonium chloride) (PDADMAC) with potassium bromide (KBr) salt, and used ion conductivity measurements coupled with UV-Vis spectroscopy to quantify the amount of salt and polymer in each phase. These experimental data were in near-quantitative agreement with Gibbs Ensemble Monte Carlo simulations using a coarse-grained coacervate model (Figure 7p), with both demonstrating the previouslypredicted partitioning of salt into the supernatant phase. ${ }^{34}$ These results followed the same trend as prior experimental data on the same system from Schlenoff et al., (Figure $7 \mathrm{k}$ ). ${ }^{50}$ In both the PRISM theory and Monte Carlo simulations, ${ }^{34 / 87}$ this partitioning was attributed to the presence of excluded volume, with the finite density of the coacervate-forming species expelling the salt ions.

This initial work by Sing and Perry prompted discussion of salt partitioning in the context of a number of theoretical, 31/31|36 87|135|167|177 computational,34|35|179 and experimental efforts. $\frac{3435}{35}$ The immediate follow-up to the Sing and Perry work was the development of the transfer-matrix theory by Sing, 31 which incorporates a phenomenological cubic term in the free energy to account for the excluded volume and packing effects in dense, coacervate systems. This theory exhibits nearquantitative matching with their prior simulation work (Figures 6d, 8, , 34 179 and extensions to multivalent ions suggest that divalent ions may alternatively lead to partitioning into the coacervate. 176 This inversion in salt partitioning was attributed to a combinatorial entropy gained by the divalent salts due to the increased number of species present in the coacervate phase.176

A parallel effort by Wang led to a new liquid state theory of coacervation, $36[167$ which provided a more nuanced prediction. Here, most of the phase diagrams are consistent with predictions of salt-partitioning to the supernatant phase, yet this inverts at very low salt concentrations (Figure 8p). 36/167 Wang attributes this observation primarily to connectivity, by comparing a series 

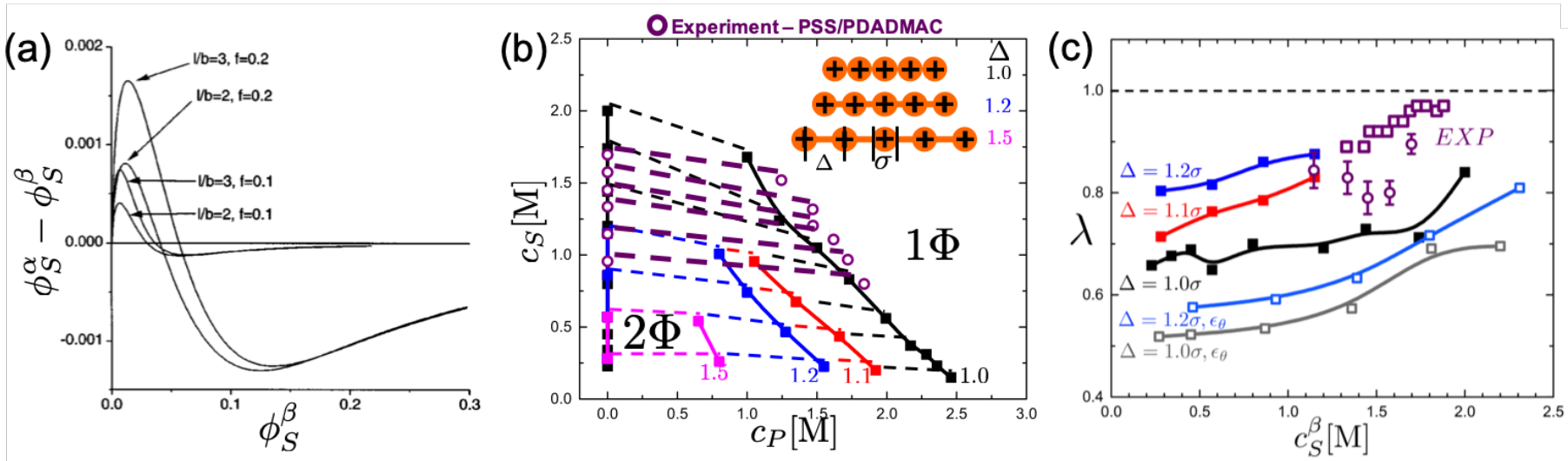

Fig. 7 Salt partitioning. (a) Prediction from Kudlay and Olvera de la Cruz ${ }^{991100}$ that the inclusion of finite-size charges leads to a partitioning of small molecule salt ions into the supernatant. Here, the plot is of the polymer-phase salt concentration minus the supernatant salt concentration $\left(\phi_{S}^{\alpha}-\phi_{S}^{\beta}\right)$ as a function of the supernatant salt concentration $\phi_{S}^{\beta}$. Reprinted from Kudlay and Olvera de la Cruz, J. Chem. Phys., 2004, 120, 404-412, with the permission of AIP Publishing. (b) Combined simulation and experiment phase diagram (salt versus polymer concentration, $c_{S}$ versus $c_{P}$ ) from Radhakrishna et al. 34 showing qualitative matching of phase behavior for PSS/PDADMAC coacervates formed in $\mathrm{KBr}$ salt solution (open symbols) and Gibbs Ensemble polymer simulations (filled symbols). Negatively sloped tie lines for both simulation and data denote partitioning of salt to the supernatant, in agreement with Kudlay and Olvera de la Cruz. Different binodal curves calculated in simulation for different linear charge spacings, and graph axes are switched from original version. 34 (c) Salt partitioning for phase diagrams in (b), measured as the ratio of salt concentration in the coacervate $(\alpha)$ and supernatant $(\beta)$ phases, $\lambda=c_{S}^{\alpha} / c_{S}^{\beta}$, versus the supernatant salt concentration $c_{S}^{\beta}$. A number of different coarse-grained representations (charge spacing $\Delta$, short-range attractions $\varepsilon_{\theta}$ ) exhibit qualitative matching with experimental data (open symbols) from Perry 34 and

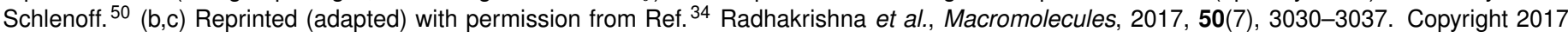
American Chemical Society.

of models with different combinations of assumptions (including Voorn-Overbeek and RPA) .36 Finally, a theory by Muthukumar, et al. predicts the partitioning of salt to the supernatant phase as well, in this case attributed to the presence of strong, favorable interactions between the dipoles formed between paired complexes and the surrounding salt ions. 135

Experimental and computational efforts further probed the Olvera de la Cruz prediction, 99100 to demonstrate results consistent with the Sing and Perry observations. One notable exam-

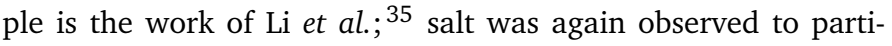
tion into the supernatant phase, in this case for a different set of coacervate-forming polypeptides (poly ( $L$-lysine) and poly $\left(D, L^{-}\right.$ glutamic acid)), using thermogravimetric analysis to calculate the coexisting salt and polymer concentrations. ${ }^{[35}$ Comparisons were also made to simulation, contrasting with the Sing results by the use of explicit solvent. $\frac{3435}{13 n t e r e s t i n g l y, ~ t h e y ~ o b s e r v e d ~ t h e ~ s a m e ~}$ salt partitioning inversion seen in Wang's theory at low salt concentrations (Figure 8k,,${ }^{36}$ in both the experiment and simulation. ${ }^{35}$ It is noteworthy that the use of explicit solvent in these simulations required the use of soft potentials, and that the form of these potentials resulted in pronounced changes in phase behavior consistent with the argument that excluded volume was key to observe partitioning of salt into the supernatant phase. 35

Despite extensive efforts to isolate the physical reasons for salt partioning, regardless of the preferred phase, there remains a degeneracy of theoretical predictions. The Wang work highlights this challenge, showing how subtle differences in model can lead to similar — but not identical — results. $\frac{36}{6}$ Ultimately, the field suffers from a dearth of data, with only a few experimental phase diagrams that explicitly consider salt partitioning. $.34 \mid 35447$ Support for these theoretical observations thus rely on only a few different chemistries, highlighting the need for more phase behavior data that includes partitioning for a wide variety of of polyelectrolyte and salt combinations. In addition to the challenges discussed thus far, related to understanding salt partitioning and the role of different polymer chemistries, the role of water in complex coacervation is a challenge that has thus far gone largely unconsidered due to the difficulties in studying such effects experimentally, as well as in theory and simulations.

\subsubsection{Polymer Stiffness and Architecture}

Most studies of coacervate phase behavior have focused on the fundamental, molecular nature of the phenomena, in particular the role of polymer connectivity and excluded volume. However, the relevance of biomacromolecules has highlighted the importance of other polymer physical attributes of the coacervateforming polyelectrolytes. In particular, biopolymers can exhibit both significant 'stiffness,' such as in DNA where the persistence length of ca. $50 \mathrm{~nm}$ is larger than the molecular dimensions of many synthetic polymers,, 180 and can also have significant molecular branching, such as in many biopolymers such as mucins ${ }^{181}$ and other glycoproteins. $\frac{182 \mid 183}{}$ Beyond simple arguments capturing the strength of electrostatic interactions and long-range conformational structures, understanding the role of stiffness and architecture require a more detailed molecular view of chargecharge correlations.

Recent efforts have begun to explore these molecular features, in both biopolymer and synthetic polymer systems. Both architecture and stiffness effects have been widely explored in the context of charged complexes, such as between DNA and branched polyelectrolytes or dendrimers, as a way to tune the interactions in polyplexes. $.133|144| 185 \mid 186$ This understanding has been extended 

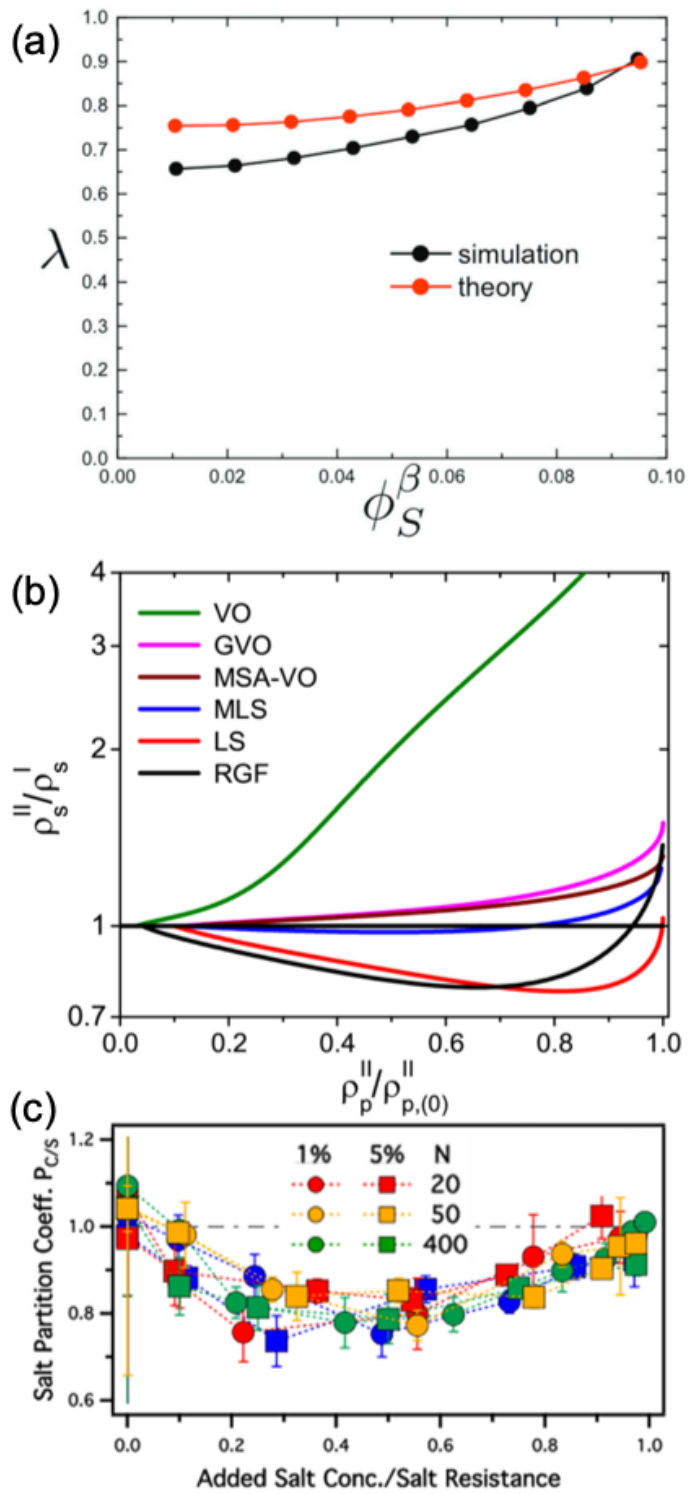

Fig. 8 Voorn-Overbeek. (a) Predictions of salt partitioning from the Transfer Matrix theory, ${ }^{[31}$ defining the salt partitioning coefficient $\lambda=$ $c_{s}^{\alpha} / c_{s}^{\beta}$ as the ratio of salt in the coacervate phase $(\alpha)$ versus supernatant phase $(\beta)$, the same as in Figure 7 k. ${ }^{34}$ For both simulation and theory, salt preferentially partitions to the supernatant phase. Ref 31 , Lytle and Sing, Soft Matter, 2017, 13, 7001-7012 - Reproduced by permission of The Royal Society of Chemistry. (b) Comparisons by Zhang et al. 36 for the same quantity, with a number of different theoretical approaches; Voorn-Overbeek (VO), generalized VO (GVO), mean-spherical approximation VO (MSA-VO), liquid state theory (LS), modified LS (MLS), and renormalized Gaussian fluctuation (RGF). Most of these are combinations of liquid state or field theory approaches, with only some exhibiting preferential partitioning to the supernatant. Reprinted (adapted) with permission from Ref. 36 Zhang et al., Macromolecules, 2018, 51(15), 55865593. Copyright 2018 American Chemical Society. (c) Experimental results from $\mathrm{Li}$ et al. 35 for a number of different polymer concentrations ( $1 \%$ and $5 \%$ ), degrees of polymerization $N$, and all normalized by the salt resistance. Similar to earlier observations,, 34 and consistent with (a) and (b), salt partitions preferentially to the supernatant. Reprinted (adapted) with permission from Ref. ${ }^{35}$ Li et al., Macromolecules, 2018, 51(8), 2988-2995. Copyright 2018 American Chemical Society.
A Single-stranded 66 nt DNA + 50 aa pLys

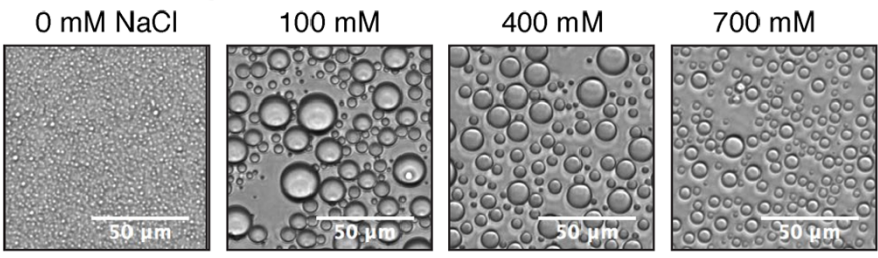

B Double-stranded 66 bp DNA + 50 aa pLys
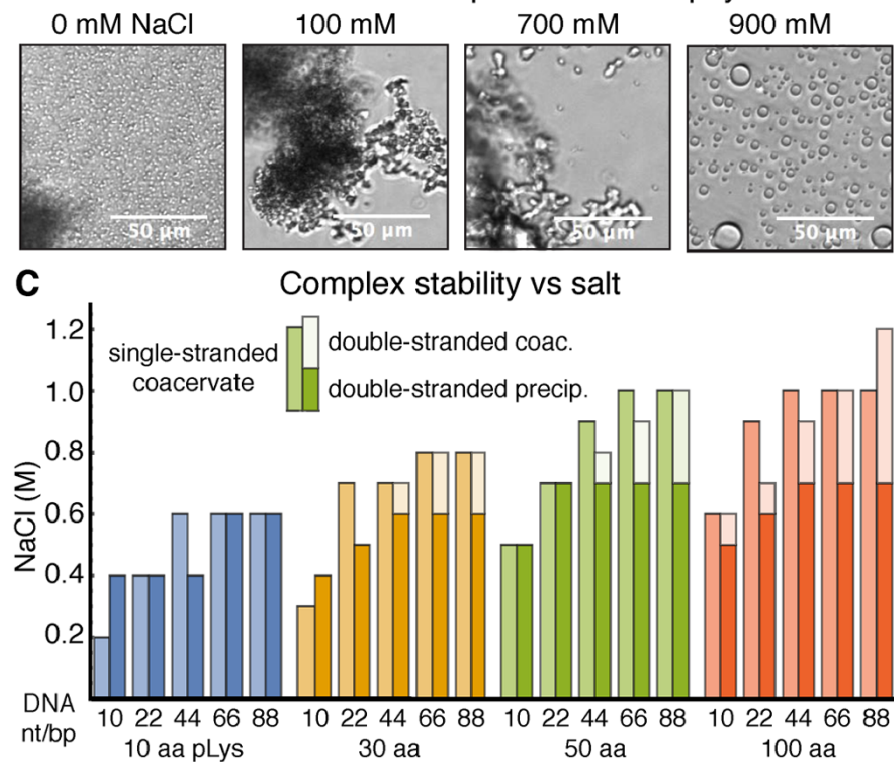

D Fraction of soluble DNA vs [NaCl]

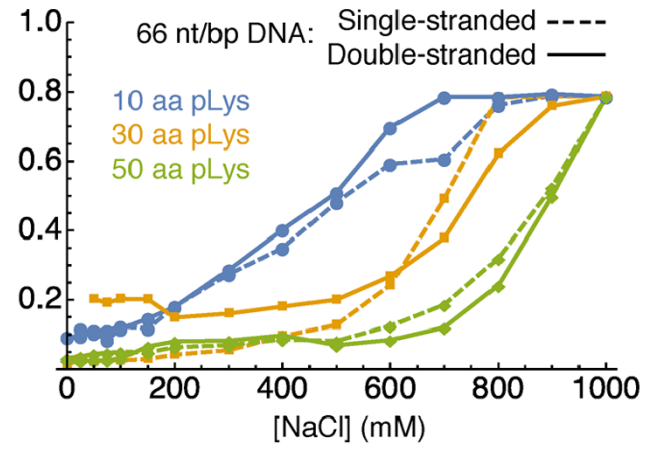

E Double-stranded 10bp DNA + 50 aa pLys, $300 \mathrm{mM} \mathrm{NaCl}$

\begin{tabular}{|c|c|c|c|c|}
\hline $49^{\circ} \mathrm{C}$ & $50^{\circ} \mathrm{C}$ & $51^{\circ} \mathrm{C}$ & $52^{\circ} \mathrm{C}$ & $53^{\circ} \mathrm{C}$ \\
\hline 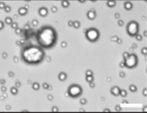 & 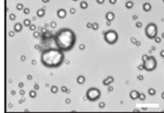 & 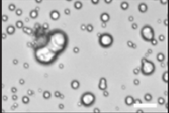 & 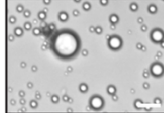 & 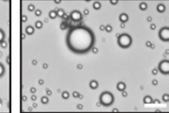 \\
\hline
\end{tabular}

Fig. 9 Polymer Stiffness. Oligonucleotide-peptide polyelectrolyte complex behavior vs. salt concentration and temperature. Representative phase contrast images of complexes formed between polylysine (pLys) and $(\mathrm{A})$ single-stranded DNA or (B) double-stranded DNA as a function of increasing salt concentration (scale bar $50 \mu \mathrm{m}$ ). (C) Phase diagram for oligonucleotide-pLys complexation. (D) Fraction of DNA complexed vs. $\mathrm{NaCl}$ concentration and polymer length for single and double-stranded DNA of length $66 \mathrm{nt} / \mathrm{bp}$. Values are normalized to the average value at $1 \mathrm{M} \mathrm{NaCl}$ to aid visual comparison. (E) Thermal melting of solid complexes of $10 \mathrm{bp}$ double-stranded DNA and pLys. (Scale bar $=25 \mu \mathrm{m}$.) Reprinted (adapted) with permission from Ref. 184 Vieregg et al., J. Am. Chem. Soc., 2018, 140(5), 1632-1638. Copyright 2018 American Chemical Society. 
to coacervates with non-linear polymer architectures, in the context of understanding the role of connectivity (vide supra) where short charged branches have been compared to analogous systems with charges along a linear polymer. The presence of short branches was demonstrated to decrease the stability of coacervates compared to their linear counterparts. 46 Combined theory, experiment, and simulation studies attributed this to a decreased 'effective' linear charge density due to the presence of only short runs of connected charges. $\underline{46176}$

Experiments have also explored the effect of stiffness and charge density, by comparing the behavior of coacervates formed from single- and double-stranded DNA. ${ }^{184}$ Here, a primary challenge is the tendency to form kinetically-trapped precipitate structures when the DNA contained more than $\sim 40 \%$ double-stranded DNA (Figure 9). The effect of stiffness is difficult to deconvolute from the differences in linear charge density, but apparent differences between stiff double-stranded and flexible singlestranded DNA coacervate phases are small and (mostly) show a decrease in coacervation with the stiff double-stranded DNA.184 This is consistent with simulation and theory that also shows that rigidity suppresses coacervate formation. 176|187 Additional experiments taking advantage of methylphosphonate substitution demonstrated that decreasing the charge density led to coacervation. 184

\subsection{Dynamics and Mechanics of Complex Coacervates}

The phase behavior and structure of coacervation has been a primary focus in the polymer physics community, however coacervate dynamics remains an emerging area that has implications for both the self-assembly and industrial application of these dense polymer solutions. In most practical cases, coacervate dynamics are inseparable from efforts to understand equilibrium properties, where there are typically questions of whether or not a coacervate system is even in equilibrium. The line between equilibrium and non-equilibrium is often determined visually, as the difference between precipitates and droplets observed in microscopy; $\underline{4450 \mid 188}$ nevertheless, recent work has begun to address the need for a more rigorous understanding of the timescales present in coacervate systems.

\subsubsection{Coacervate Rheology and the Complex/Coacervate Continuum}

Reports on the phase behavior of complexation have described the occurrence of both liquid complex coacervates and solid precipitation, though the terminology is frequently not always clearly defined. 44 The formation of solid precipitates has largely been considered unfavorable because of the intractability of these materials to further processing via traditional methods such as solvent or temperature. ${ }^{189}$ This has long motivated speculation on the relationship between solid precipitates, liquid complex coacervates, and other forms of polyelectrolyte complexes such as layer-bylayer (LbL) films, and recent efforts have attempted to better eludicate these relationships. In particular, a seminal report by Wang and Schlenoff demonstrated that solid precipitates and liquid coacervates could exist along a continuum of salt concentration (Figure $10, .50$ This idea of salt-driven plasticization, or 'salo-

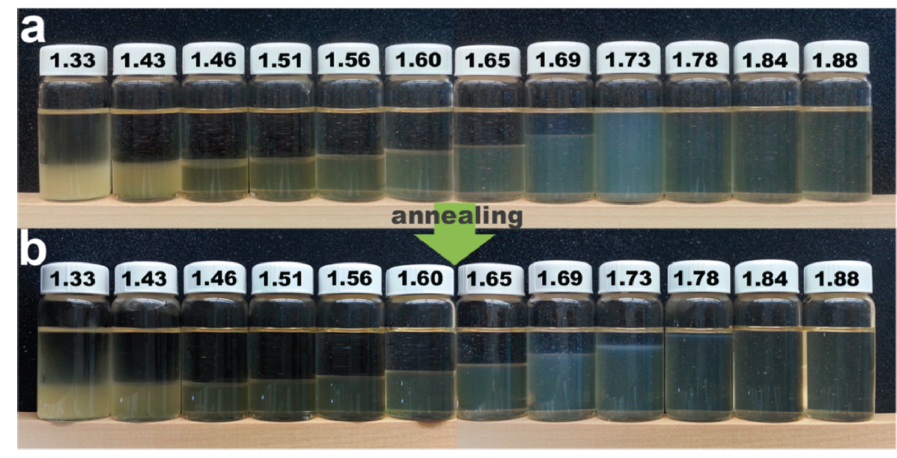

Fig. 10 Solid-to-Liquid Continuum. Photographs of (a) as-prepared poly(4-styrene sulfonic acid sodium salt)/poly(diallyldimethylammonium chloride) (PSS/PDADMAC) coacervate samples stored for 30 days and (b) the samples 10 days after annealing for about $3 \mathrm{~h}$ at $60^{\circ} \mathrm{C}$ and cooled to room temperature. The numbers indicate the concentration of $\mathrm{KBr}$ (in $\mathrm{M}$ ) for each sample. Increasing water content is observed in the coacervate phase up to $1.80 \mathrm{M} \mathrm{KBr}$, above which, only a single solution phase is observed. Reprinted (adapted) with permission from Ref. 50 Wang and Schlenoff, Macromolecules, 2014, 47(9), 3108-3116. Copyright 2014 American Chemical Society, https://pubs.acs.org/doi/abs/10.1021/ma500500q. Further permissions related to the material excerpted should be directed to the ACS.

plasticity' has opened up a tremendous range of opportunities for using salt to process polyelectrolyte complex materials as liquid coacervates, and then solidify the materials by the removal of salt, and a range of accounts describing ultracentrifuged 190 or extruded materials, 194 spin-coated films, $195 \mid 196$ 3D printed structures, 197 and electrospun fibers $65[198 \mid 199$ have been reported.

\subsubsection{Understanding the Role of Salt and Water on Coacer- vate Mechanics}

Studies aimed at understanding the rheology of complex coacervates have focused on the linear viscoelastic response, and interpreting molecular, coacervate dynamics in the context of macroscopic phase behavior (and thus the composition of the material). Here we will discuss three separate models that have been used to describe the rheology of coacervate-based materials, (i) the idea of time-salt superposition, (ii) the importance of water content, or the swelling, of the material, and (iii) the relationship between the water content and the number of ion pairs present.

Time-Salt Superposition: Oscillatory shear rheology experiments typically used to study the mechanics of viscoelastic samples are only able to access a relatively narrow range of frequency space. Superposition principles have long been used as a tool in rheology to access a range of timescales that would otherwise be out of experimental reach by using an experimental variable such as temperature or strain rate as a means for accelerating activated processes. 58 In the context of complex coacervates, Spruijt et al. demonstrated the idea of a time-salt superposition (Figure 11 . 3757

Time-salt superposition of linear viscoelasticity data for complex coacervates can be achieved by shifting individual frequency sweep curves with respect to a reference condition (Figure 11, b). The data are typically shifted both horizontally and vertically. The vertical shift factor $G_{c}$ serves as a correction to the moduli 

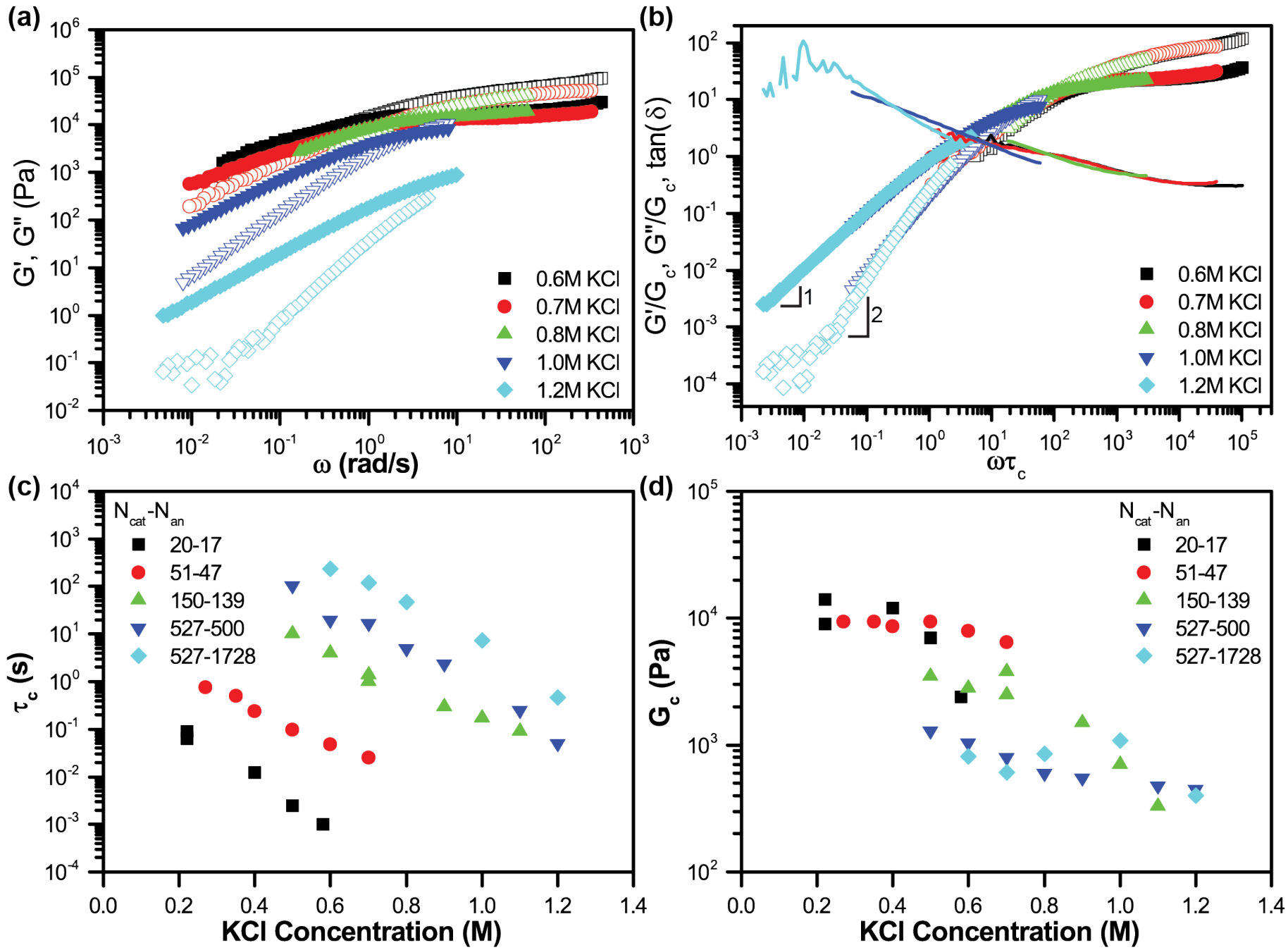

Fig. 11 Time-Salt Superposition. (a) Frequency sweep data for stoichiometric poly(N,N-dimethylaminoethyl methacrylate)/poly(acrylic acid) (PDMAEMA/PAA) coacervates prepared in $\mathrm{KCl}$ solutions at $\mathrm{pH} 6.5$ with an average degree of polymerization of $\mathrm{N}=527$ for PDMAEMA and $\mathrm{N}=$ 1728 for PAA. The storage modulus $G^{\prime}$ is shown in open symbols, the loss modulus $G^{\prime \prime}$ is shown in closed symbols. (b) A rescaled time-salt superposition of the data from (a) where the frequencies have been rescaled using a salt-dependent shift factor $\tau_{c}$ shown in (c), and the storage (open) and loss moduli (closed) have been rescaled using a salt-dependent shift factor $G_{c}$ shown in (d). The continuity of the tan $(\delta)$ plot (line) demonstrates the quality of the superposition. The crossover between $G^{\prime}$ and $G^{\prime \prime}$ was taken as the reference condition to define $\omega \tau_{c}=1$ and $G^{\prime} / G_{c}=1$. Figures adapted

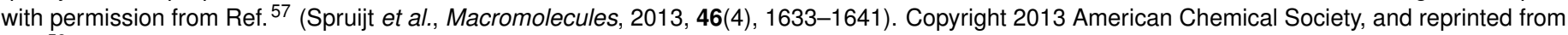
Ref. ${ }^{58}$ Adv. Colloid Interface Sci., 239, Liu, Winter, and Perry, Linear Viscoelasticity of Complex Coacervates, 46-60, Copyright (2017), with permission from Elsevier. 
$\left(G_{\text {scaled }}^{\prime}=G^{\prime} / G_{c}\right.$ and $G_{\text {scaled }}^{\prime \prime}=G^{\prime \prime} / G_{c}$ ) to account for differences in the polymer concentration in the coacervate at the different salt concentrations (Figure 11d). 37/57 The horizontal shift factor $\tau_{c}\left(\dot{\gamma} \rightarrow \dot{\gamma} \tau_{c}\right)$ accounts for changes in the stress-relaxation behaviour of the material, which is attributed to the weakening of electrostatic interactions via charge screening (Figure 11k). 3757 This is analogous to the traditional time-temperature superposition, which has also been reported for coacervates ${ }^{53[59}$ and polyelectrolyte complexes, 200 except that salt concentration weakens interactions (and expedites dynamics) rather than temperature.

The approach by Spruijt et al. describes the stress relaxation of the polymer chains in the coacervate in terms of a "sticky" Rouse model, 37/57201 where electrostatic interactions between chains act as "sticky" points that enhance the effective friction of polymer chains, slowing their relaxation. The breaking of these "sticky" points is related to the dissociation rate of the ion-ion pairs, and was described as an activated process governed by an energy barrier:

$$
\tau_{0}=\frac{1}{\omega_{0}} \exp \left[\frac{E_{a}\left(c_{\text {salt }}\right)}{k_{B} T}\right]
$$

where $E_{a}$ is estimated as the difference between the electrical free energy of four separated ionic groups in an electrolyte solution, and the Coulombic energy of two ion-ion pairs in contact, as described by the Debye-Hückel approximation.

$$
\tau_{c} \propto \frac{1}{\omega_{0}} N^{\alpha} \phi_{p}^{\beta} \exp \left[-a(T) \sqrt{c_{\text {salt }}}+\lambda_{B} / d\right]
$$

This time scale $\tau_{c}$ is related to an attempt frequency $\omega_{0}$, the degree of polymerization $N$ and polymer concentration $\phi_{p}$ (with exponents $\alpha$ and $\beta$ ), $a$ is a constant defined in Ref. $\frac{37}{\text {, }}$, and $d$ is the distance between two charges at contact. The physical argument for the energy stem stems from the difference of energy at contact $\left(\sim k_{B} T \lambda / d\right)$ and the electrostatic energy for unconnected charges $\sim k_{B} T \lambda \kappa$ Thus, this model predicts a square root dependence of the activation energy with respect to salt concentration $\sqrt{c_{\text {salt }}}$. $37 / 60$ Hamad et al. later extended this result, considering that cooperativity between polymers would result in a characteristic "sticky" point involving interactions from a cluster of cooperative charges. By including the number of individual ionic interactions within a characteristic "sticky" point, the authors were able to collapse shift factor data from polymers of different chain lengths onto a single, universal curve. 60

$$
\tau_{c} \propto \frac{1}{\omega_{0}} N^{\alpha} \phi_{p}^{\beta} \exp \left[-n\left(a(T) \sqrt{c_{s a l t}}+\lambda_{B} / d\right)\right]
$$

where $n$ is now the number of cooperative charges, for which the authors found $n=5.60$ Only a single simulation paper by Andreev et al. has sought to reproduce this result, 202 reproducing the effect of time-salt superposition and experimental $G^{\prime}$ and $G^{\prime \prime}$ curves; however, the trends in shift factors were not observed to match with experimental values. $\frac{202}{20}$ This was attributed to the need to include more atomistic detail beyond the coarse-grained model used in simulations, especially the effect of ion hydration. 202

While the majority of reports have focused on the rheological response of liquid coacervates, an analysis by Liu et al. examined changes in the linear viscoelasticity of complexes as salt concen- tration was changed to convert the material from a liquid to a solid state. ${ }^{47}$ The decrease in salt concentration and the commensurate decrease in water content of the coacervates was shown to result in the formation of a physical gel, where a network of trapped electrostatic crosslinks percolates the sample at a critical salt concentration.

Time-salt superposition master curves can be created for liquid and solid samples on either side of the critical gel point; 47 however, superposition requires that the material be self-similar across all of the conditions. The formation of a percolated network at the critical gel point results in a divergence in the relaxation behavior at the critical gel point. Thus, while the modulus of a saloplastic material may continue to smootly increase with decreasing salt/water concentration, $\sqrt[53]{5}$ one should not perform a continuous time-salt superposition across the gel point. 47

The Effect of Swelling: One of the challenges of studying coacervate dynamics is the need to separately characterize the phase behavior and rheology. This requirement would typically necessitate separate measurements; however, Shull and coworkers used a quartz crystal microbalance with dissapation (QCM-D) methodology to simultaneously characterize the swelling behavior of a thin polyelectrolyte complex film, along with the modulus and phase angle of the material. ${ }^{53}$ While studies had previously demonstrated that the choice of salt could dramatically affect the phase behavior of coacervates, 45 the use of QCM-D enabled the authors to demonstrate that changes in the mechanical properties of the film were dictated by the degree of swelling in the film, rather than the identity of the salt used (Figure 12 a-c).

Water Content Per Ion Pair: As was mentioned in the context of time-salt superposition between liquid and solid polyelectrolyte complex materials, a critical aspect of any superposition is the requirement that the structure of the physical network must be unchanged. This requirement raises particular questions in the context of the swelling argument presented by Sadman et al., as swelling can be due to both salt and water, $194 \mid 204$ particularly in water limited regimes that can result in solid polyelectrolyte complexes, such as layer-by-layer films. 203

In the context of these water-limited materials, the internal structure implied by the hydrated electrostatic interactions described as "sticky" points in the context of the linear viscoelasticity of complex coacervates can better be described as a combination of intrinsic and extrinsic ion pairs. $\frac{190 / 194}{10}$ Intrinsic ion pairs represent electrostatic interactions between oppositely-charged polymers, while extrinsic ion pairs can form between a polymer and a free ion in solution. Thus, increasing the concentration of salt in the sample would be expected to increase the number of extrinsic ion pairs due to competitive binding. 194/204

Most of the discussion surrounding the idea of intrinsic ion pairing and material properties of polyelectrolyte complexes has been focused on understanding the presence of a glass transitionlike thermal transition.194/203|205|209 Lutkenhaus and coworkers explored this effect using kinetically trapped polyelectrolyte complex solids and multilayers where the water and ion content of the material could be varied independently to explore the effect of these parameters on the resulting material properties. Experimental results from differential scanning calorime- 


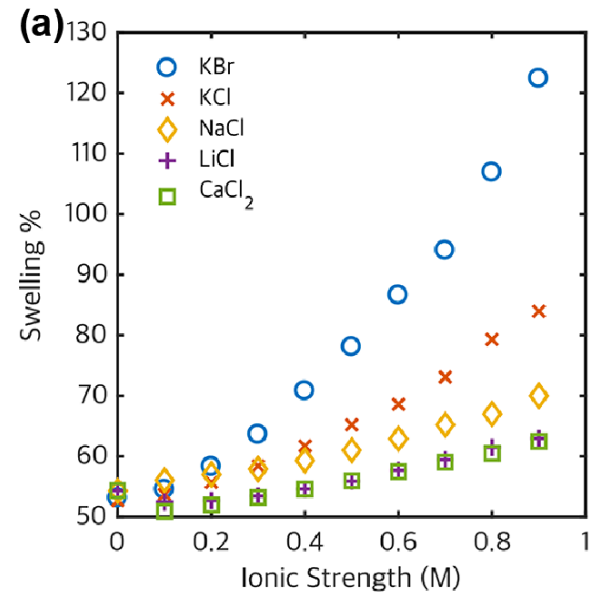

(d)

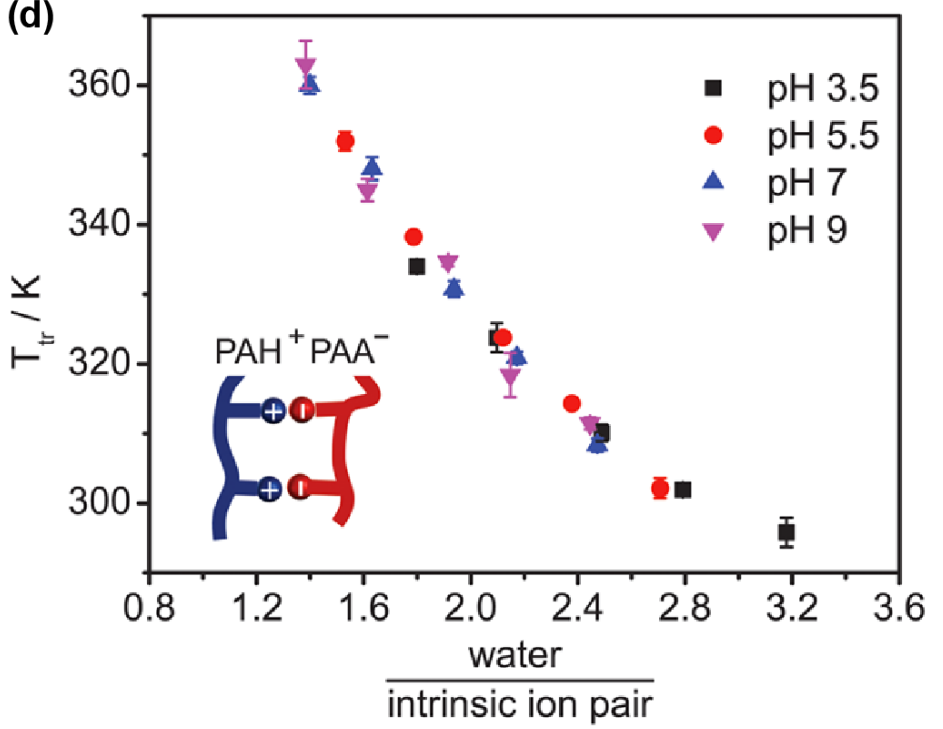

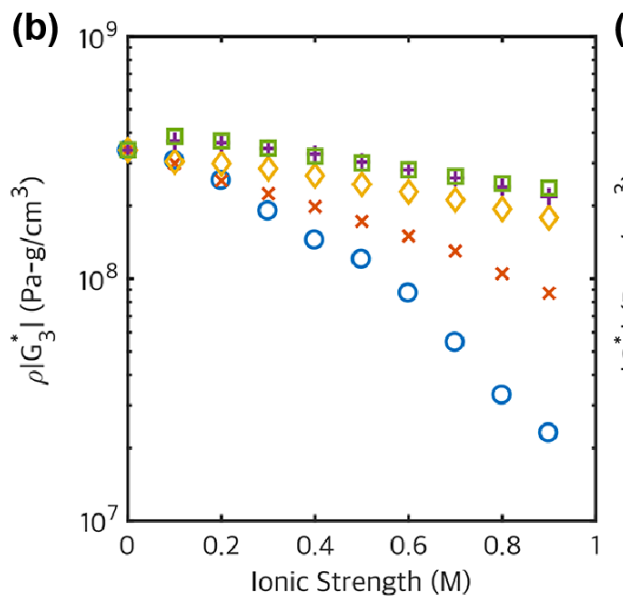

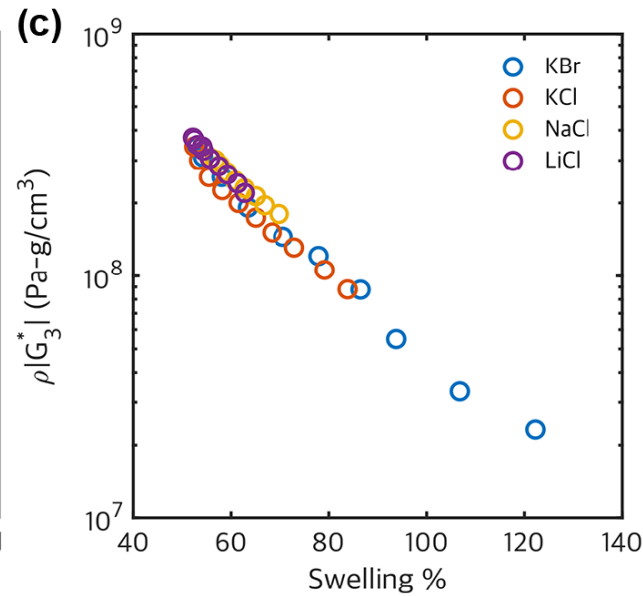

(e)

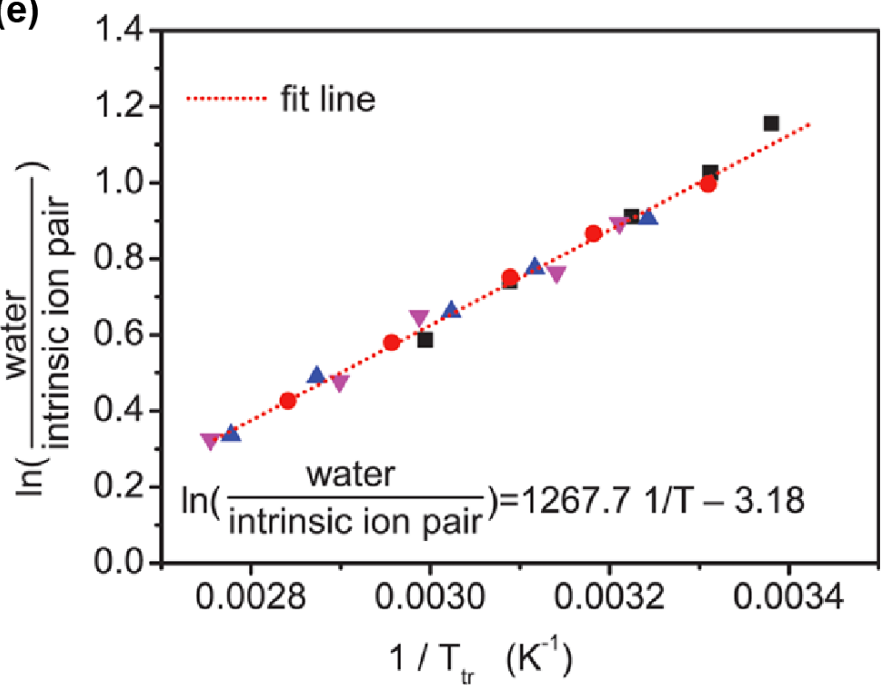

Fig. 12 Effect of Swelling. (a) Swelling behavior calculated in reference to the dry film and (b) density-shear modulus as a function of the solution ionic strength and salt identity for spin-coated PSS/PDADMAC films. (c) The combined graph of density-shear modulus vs. \% swelling shows near universal collapse of the data from different salts. (a-c) Reprinted (adapted) with permission from Ref. ${ }^{[53}$ Sadman et al., Macromolecules, 2017, 50(23), 9417-9426. Copyright 2017 American Chemical Society. (d) Thermal transition temperature $\left(T_{t r}\right)$ vs. the number of water molecules per intrinsic ion pair in hydrated poly(allylamine hydrochloride)/poly(acrylic acid) (PAH/PAA) complexes prepared at pH 3.5, 5.5, 7, and 9. The number of water molecules was taken as the total amount water added to the complex. The number of intrinsic ion pairs was calculated from the mass and polymer composition of the complex, assuming that all PAH units were ionized and participate in intrinsic ion pairing. (e) Linear fitting of In(water/intrinsic ion pair) vs. $1 / T_{t} r$. The legend in (d) also applies to (e). (d,e) Reprinted (adapted) with permission from Ref. ${ }^{203}$ Zhang et al., Macromolecules, 2016, 49(19), 7563-7570, Copyright 2016 American Chemical Society, https://pubs.acs.org/doi/10.1021/acs.macromol.6b00742. Further permissionsrelated to the material excerpted should be directed to the ACS. 
try (DSC), thermogravimetric analysis (TGA), electrochemical impedance spectroscopy (EIS), and dynamic mechanical testing (DMA) were compared by molecular dynamics (MD) simulations by Sammelkorpi and coworkers. $63 \mid 200 / 203 / 210$ These reports ultimately determined that the temperature of the thermal transition between samples prepared at different water, salt, $\mathrm{pH}$, solvent, and other additive contents could be collapsed onto a universal curve by normalizing based on the water concentration per intrinsic ion pair (Figure 12 d). 203206 Furthermore, it was possible to linearize this universal curve by plotting the natural log of the ratio of (water/intrinsic ion pair) as a function of $\left(1 / T_{t r}\right)$ (Figure 12 ). These results were further extended to show that it was possible to perform both a time-temperature and a time-water superposition at constant salt because the variation in the intrinsic ion pair concentration was minimal. 200

Ultimately, these three areas of study into coacervate dynamics consider a wide range of time/length scales; the work on glass transition-like thermal transitions considers local segmental and water dynamics, 194/203/205,209] while the time-salt superposition work borrows from the 'sticky Rouse' theories that consider the dynamics of the entire chain conformation. 37/57/201 These areas of recent progress thus remain only brief glimpses at a rich dynamic behavior of coacervate dynamics and rheology, and suggest the importance of hydration and other atomistic-level effects in the practical application of these materials.

\subsection{Hierarchical Coacervate Self-Assembly}

Coacervate-driven phase separation, similar to $\chi$-driven phase separation, $211 \mid 212$ can drive nanoscale assembly in block copoly-

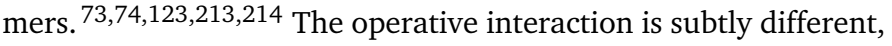
in that instead of a dislike of two different species, phase separation occurs due to the association of two oppositely-charged species. There are typically two polymeric species; one polyanion and one polycation, where at least one of the species is a block copolymer with a single neutral block. ${ }^{73}$ The requirement of a binding partner can complicate coacervation, along with the importance of electrostatic interactions. Another key difference is the aqueous nature of both phases in a self-assembled system, which has ramifications for applications in e.g., drug delivery or encapsulation, 6/76/215 where it may be desirable to incorporate a hydrophobic or a hydrophilic/charged species into the center of a micelle or other self-assembled structure. Thus, concomitant with efforts to understand the fundamental phase behavior of bulk coacervates, there have been extensive studies on how coacervate-driven self-assembly occurs in polyelectrolyte block copolymers. 73

\subsubsection{Phase Behavior in Coacervate Assembly}

Early efforts focused on the formation of 'coacervate-core micelles', 73/75/77/213|214/216|218 which have particular relevance to drug delivery technologies. Extensive reviews have covered early work on these systems, 73 and highlight the abundance of material systems considered and the micellar morphologies that can be obtained. Indeed, it is arguable that at some point the promise and interest in coacervate-core micelles led the state of understanding in these systems to surpass even bulk coacervation; a richer array of different polyelectrolytes, combined with a wider exploration of the parameter space, resulted in questions that have been discussed only in the past few years for bulk coacervation. ${ }^{73}$ What is the nature of kinetic trapping versus liquid-like coacervation in the micelle core? ${ }^{45|219| 220}$ What are the ramifications of nonstoichiometric charge on assembly? ${ }^{221 \mid 222}$ How do we rationalize the phase behavior of micelles with repsect to the electrostatic attractions versus the role of counterion entropy? ${ }^{223}$ Nevertheless, the answers to these questions have long been stymied by a lack of understanding in bulk coacervation, a dearth of theoretical modeling and theory for coacervate-core micelles, and the extent that potential applications focused work almost exclusively on dilutesolution micelles. ${ }^{73 / 224}$ Ultimately, more recent efforts have gone a long way in considering these questions as the field has begun to fill in a more fundamental picture of coacervation.

Systematic experiments on coacervate block copolyelectrolyte self-assembly have considered both diblock and triblock copolymers, and used X-ray and neutron scattering to map out the ordered morphologies that occur at increasingly high block copolymer concentration. A series of papers show the formation of ordered morphologies such as BCC-packed micelles and hexagonally-packed cylinders, along with a few examples of lamellae or gyroid phases. $82[86|123| 179|225| 226$ These morphologies correspond to those found in traditional $\chi$-driven selfassembly, and it would be unsurprising if more elaborate phases seen in $\chi$-driven systems are similarly observed in the future for coacervate-driven assembly.

A phase diagram for diblock coacervate-driven assembly has been determined for short charged blocks, in the salt versus polymer concentration plane, and compared directly with triblock copolyelectrolyte assemblies (Figure 13a). 83|84|86|225 The features of these self-assembled structures were further characterized by cryo-TEM (Figure $13 \mathrm{p}$ ). .85 The latter was actually better explored, due to the ability of triblocks to assemble into gels. $\frac{83}{}$ Here, the end-blocks are charged and form coacervatebased 'crosslinks' connected by neutral mid-blocks. The desirable property of these gels is as a highly tunable soft material, and a number of papers have considered their mechanical, assembly, and phase behavior over a number of molecular parameters (e.g., block fraction, salt concentration, polymer concentration). $83|84| 86|218| 225|227,230|$ In particular, it has been recently shown that these gels form for triblocks at extremely low concentrations, phase-separating to form a percolated network. 83

Simulation and theory have also been developed to explain some aspects of coacervate solution self-assembly, however there still remain practical challenges to determining the suitability of these observations. Perhaps the earliest prediction was from Audus et al. ${ }^{82}$ which used the one-loop RPA theory of Castelnovo and Joanny 116 as the input to a self-consistent field theory model, in a method dubbed the 'embedded fluctuation model'.82 This was performed for triblock copolyelectrolyte assembly, and exhibited qualitative matching with an experimentally-determined phase diagram in the low-salt limit (Figure 13 f). ${ }^{82}$ More recently, a scaling approach has considered the assembly of coacervatecore micelles, providing predictions for morphology transitions in dilute solutions of block copolyelectrolytes (Figure 13d). 123 

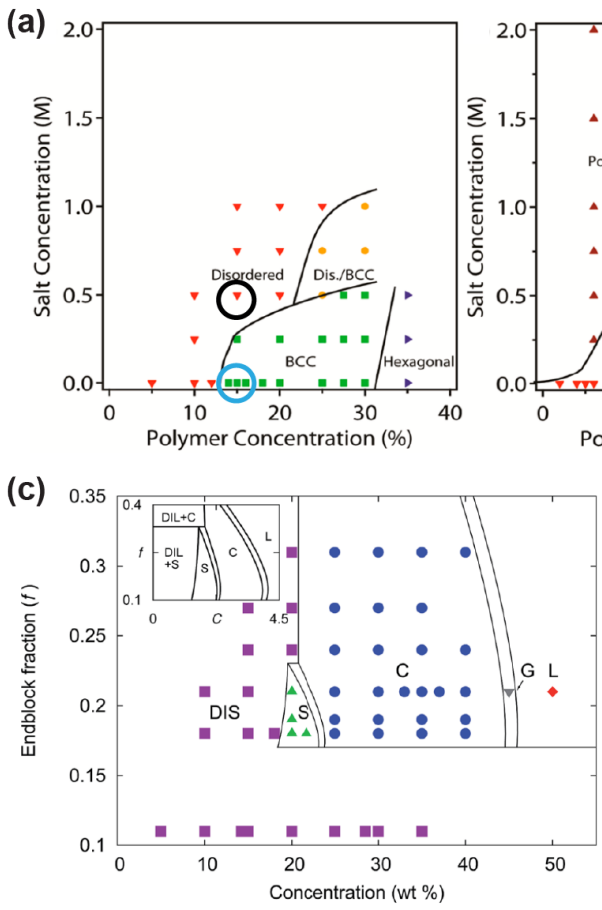

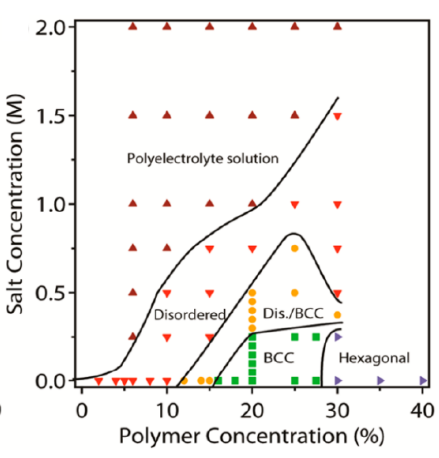

(d) (b)

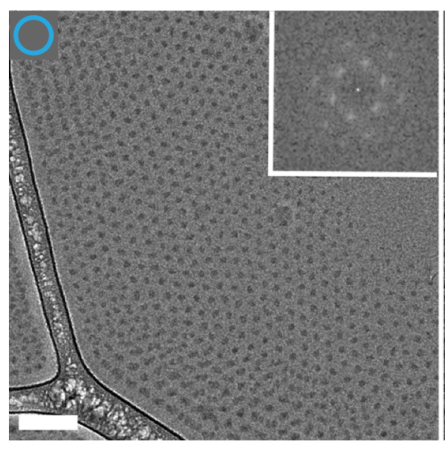

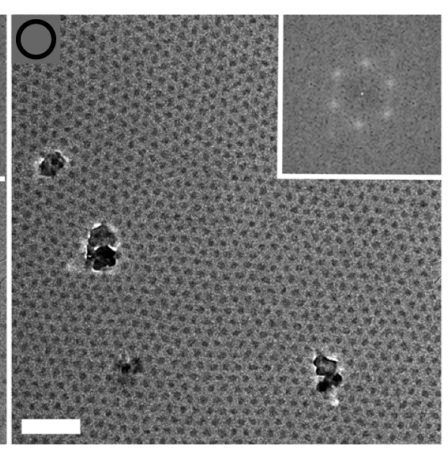

(e)

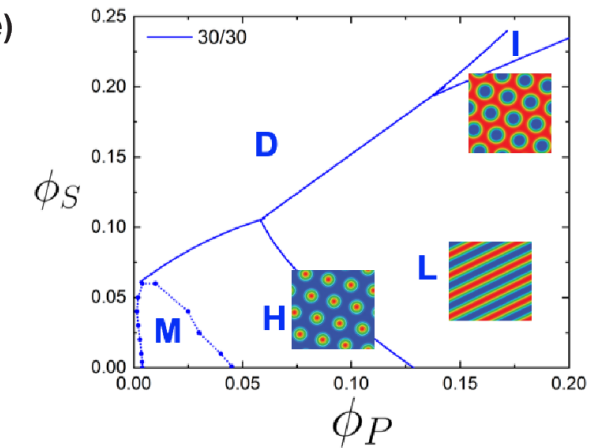

Fig. 13 Coacervate Self-Assembly. (a) Work from Krogstad et al.,, 86 mapping the self-assembled phase behavior of diblock (left) and triblock (right) block copolyelectrolytes. Disordered micelles, BCC-packed micelles, and hexagonally-packed cylinders are all observed. Reprinted (adapted) with permission from Ref. ${ }^{86}$, Krogstad et al., Macromolecules, 2014, 47(22), 8026-8032. Copyright 2014 American Chemical Society. (b) Self-assembled morphologies were characterized by cryogenic tunneling electron microscopy (cryoTEM), with circles in upper left corresponding to points on the phase diagram in (a). Reprinted (adapted) with permission from Ref. 85 , Krogstad et al., J. Phys. Chem. B, 2014, 118(45), 13011-13018. Copyright 2014 American Chemical Society. (c) Audus et al. developed the embedded fluctuation model to predict phase diagrams (inset) that match with experimental data for triblock coacervate self-assembly. Reproduced from Ref. 82 with permission from The Royal Society of Chemistry. (d) Scaling theory has been used to predict the transitions among different micelle structures in the low polymer concentration limit. Reprinted (adapted) with permission from Ref. 123, Rumyantsev et al., ACS Macro Lett., 2018, 7(7), 811-816. Copyright 2018 American Chemical Society. (e) Phase diagram calculated by Ong and Sing using hybrid transfer matrix-SCFT calculations. 226 This example is performed in two-dimensions, capturing similar phase behaviors to those seen in both (a) and in the uncharged self-assembly literature. ${ }^{231232}$ Reproduced from Ref. ${ }^{226}$, with permission from The Royal Society of Chemistry. 
While consistent with other scaling models of complex coacervation, $107 / 108 / 120$ these predictions remain to be experimentally verified.

Both the scaling and embedded fluctuation predictions have focused on fluctuation-driven coacervation, suitable for low chargedensity systems. Recently, the opposite limit of high chargedensity has also been incorporated into self-consistent field theory, ${ }^{[226}$ in a manner analogous to the embedded fluctuation approach. Here, the model for charge correlations uses the transfermatrix approach,, 31 and was used to predict the self-assembled structures for a wide range of polymer block fractions, and salt and polymer concentrations (Figure 13 e). 226 This predictions focused on two-dimensional assembly, but nevertheless determined phase behaviors similar to those found in uncharged, $\chi$-driven assembly.231.234 The primary difference here is that the temperature is replaced by salt concentration as the parameter that 'weakens' the driving force for self-assembly. 226 An extension of this theory is capable of capturing the transition from sequencedependent macro-phase separation ${ }^{137}$ to macrophase separation as blockiness increases. 235

Except for the work by Audus,, 82 there has been very little comparison with experiment for most of these theoretical predictions. However, comparisons have been made to simulation, $\underline{83}$ using a coarse-grained model analogous to simulations for bulk coac-

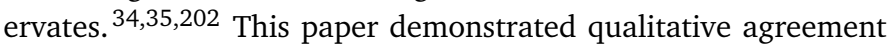
between experiment and simulation in the differences in phase behavior for diblock and triblock coacervate self-assembly. ${ }^{83}$ This represents an opportunity for theory, which has yet to be connected to these specific observations.

\subsubsection{Dynamics of Block Copolymer Assemblies}

Despite progress in understanding coacervate-driven selfassembled phase behavior, comparison between experiment and theory is stymied by the presence of long-lived, kineticallytrapped states. This challenge is similar to the issue of liquid coacervate vs. polyelectrolyte complex solids in bulk coacervates, and has been increasingly appreciated in the community, leading to a concerted effort to characterize the dynamics of structure formation and approach to equilibrium in coacervation-driven selfassembly. ${ }^{86}$ At the very least, a deeper understanding of time scales in coacervate assembly will allow the categorization of selfassemblies as in equilibrium or not; however, application of these systems will also likely rely on their dynamic characteristics (for example) in drug delivery or as stimuli-responsive materials.

\section{Modern Directions in Coacervation}

Recent interest in coacervation as a phenomena has led to deep questions that influence - and are influenced by - other emerging areas in polymer physics, materials engineering, and biophysics. In the scope of these larger trends in soft matter, it is apparent that the electrostatic interactions that drive complex coacervation are broadly relevant due to their sensitivity to molecular structure and chemical identity. Biology, in particular, makes use of this interaction motif to great effect. The outlook of coacervation thus exists at the interface between polymer science, molecular engineering, and biology, and we highlight a few directions that show particular promise.

\subsection{Connection to Layer-by-Layer Films}

The associative electrostatic interactions driving complex coacervation are an obvious parallel to those used to drive the formation of layer-by-layer (LbL) films. 236|238 While the field has long been cognizant of these parallels, 2239|240 the difficulties associated with predicting the dynamics of polyelectrolyte complex systems has limited the amount of progress that has been made. Recent experimental work by Salehi et al. has suggested a possible correlation for predicting the growth rate of LbL films based on measurements of coacervate phase behavior. ${ }^{241}$ However, further validation of this approach with a broader range of polymer systems is still needed.

\subsection{Sequence Control and Intrinsically-Disordered Proteins}

Coacervation, in a reductive sense, is merely a phase separation; much of the research in the field has significant connection with 'traditional' $\chi$-driven polymer-solvent phase separation where salt concentration takes the place of temperature. This is apparent both in bulk coacervation and in coacervate-driven assembly, with nuanced differences associated with the multi-component and electrostatic nature of the phenomenon. However, it is becoming apparent that the biological world leverages these subtleties in (for example) creating functional compartments in the cell. $18 \mid 21242$ Here, the ability of biology to precisely tune the sequence of monomers along proteins and other biomacromolecules seems to play a key role in biological function, even in highly disordered systems similar to the types of polymer solutions found in non-biological coacervation. 4 Understanding and mimicking these systems has thus become an active area of research, due not only to the impliciations in biophysics, but in a more fundamental aspiration to control polymer structure and function via monomer sequence. 252

\subsubsection{Intrinsically-Disordered Proteins}

Proteins and protein-segments that do not spontaneously fold into stable structures, called intrinsically-disordered proteins (IDPs), are known to form functional droplets in the cell that can compartmentalize and sequester biomolecules for participation in biochemical reactions. $18,21|242| 244 \mid 251$ These functional and dynamic structures, called membraneless organelles or biomolecular condensates, 253 have been the focus of extensive research in the biophysics literature. In particular, they are notable for their ability to dynamically form as needed to participate in biological processes, 253 and are thus responsive to both the concentration of components and the charged/crowded environment. $242|243| 254 \mid 257$

Significant experimental, computational, and theoretical effort has probed how the distribution of amino acids reflects in the ability of IDPs to form liquid-liquid phase separated droplets. $19|20| 22|246| 258 \mid 264$ Currently, evidence points to the critical role of monomer sequence in determining phase behavior, manifesting in a number of different ways. First, the general abundance of charged amino acids prevents folding into close- 
packed structures. The ability to phase separate then follows from the particular non-electrostatic interactions governing the interactions between IDPs and their surroundings. However, the precise sequence of positively- and negatively-charged monomers has been shown to be a key parameter in the phase behavior of these intracellular systems. 19 20|22

The importance of charge sequence was highlighted in a study by Pak et al., looking at the phase separation of the negativelycharged nephrin intracellular domain (NICD). 22 Experiments demonstrated that phase separation of NICD required both complexation with a positively charged partner, to weaken the electrostatic repulsion and facilitate phase separation, along with further stabilization by shorter-range interactions involving aromatic and hydrophobic residues. The effect of sequence was explored using mutagenesis to alter the distribution of charged and hydrophobic residues. Interestingly, the sequence of hydrophobic residues had little effect on the resulting phase behavior of the coacervate, while the distribution of charged amino acids had a significant effect.

Theory and simulation have been developed to provide insight into sequence effects in IDP-based liquid-liquid phase separation. 258 265 Efforts initially focused on single-chain properties, in an attempt to correlate IDP conformation to sequence-related parameters such as the sequence charge decoration parameter (SCD),,$\sqrt{266}$ the fraction of charged residues (FCR),, 262 and patterning parameters that relate the 'blockiness' of a charged IDP. 265 Simulation was able to show conformations ranging from random coils to disordered globules, and connection to liquid-liquid phase separation was implied. 262 More recently, this connection has been made more concrete in a number of papers using coarsegrained simulations relating single-chain conformations to bulk liquid-liquid phase separation, 259260 showing this to generally be the case (Figure 15a). Alternative approaches have considered RPA-based theories to explore the role of IDP sequence, and show that with appropriate choice of parameters it is possible to qualitatively predict some sequence trends (Figure 15b). 258/263|264 These key advances in IDP biophysics have necessarily focused on leveraging coarse-grained models or theories to capture liquidliquid phase separation, with a need to parameterize their connection to biologically-relevant sequences. This suggests a convergence with the polymer physical approaches considered in the next section, which similarly relies on a non-atomistic picture of polyelectrolyte thermodynamics.

\subsubsection{Polyelectrolyte and Polyampholyte Sequence}

Inspired by this effort to understand sequence effects in IDPs, and the recent focus in the biophysical community on using models increasingly similar to those routinely used in polymer physics, 259260|262 265 polymer physics has begun to focus on the role of sequence in non-biological settings. The promise of harnessing sequence effects to control polymer thermodynamics has prompted a number of investigations into a general polymer physical understanding of sequence-defined coacervation. $70|105| 106|121| 137|235| 267 \mid$ This builds on a larger polymer physics literature interested in sequence-defined polymers, with useful material properties emerging from (for example) multi- block copolymers, $\stackrel{268}{26 r a d i e n t}$ and tapered polymers, 269 and precision ionomers. 270271 This recent literature also follows work on understanding the role of sequence on the stability of pairwise polyampholyte/polyelectrolyte complexes, $272+274$ which is distinct from the desire to understand bulk coacervate phase behavior.

Systematic variation of sequence in coacervate-forming polymers was performed by Perry and coworkers, using homopolymers of poly(glutamate) and sequence-defined, cationic poly(glycine-co-lysine). ${ }^{[70}$ The copolymer sequence was varied from alternating charge/neutral residues to regular blocks as large as eight monomers long. The stability of the coacervate, defined as 'salt resistance,' or the concentration of salt needed to destabilize phase separation, increased with blockiness, along with the magnitude of the entropic driving force for coacervation as measured by calorimetry. These results were qualitatively consistent with simulation phase diagrams and calculations of coacervate formation. Follow-up work extended the transfer-matrix theory of Lytle and Sing to provide predictions of a number of non-regular sequences using the same sequence-defined polymer systems (Figure 14, 137 They were able to demonstrate matching between simulation, theory, and experiment, even with subtle monomer-level changes to sequences that otherwise exhibited the same charge fraction and average charge blockiness. The same team demonstrated qualitative matching was also observed for self-coacervation of a variety of sequence-defined polyampholytes, once again using polypeptides (Figure 15, d).138

Alternative theoretical approaches have also been considered to understand the role of polyelectrolyte sequence in coacervation, especially for sequence-defined polyampholytes due to their close connection to biophysical IDP systems. Danielsen and coworkers used the field theoretic framework, $\frac{104}{10}$ in close connection with MD simulations, to understand the role of sequences of polyampholyte self-coacervation. $105[106]$ They were able to show how a number of sequence effects, such as blockiness or charge asymmetry, couple with changes in the strength of electrostatic interaction and excluded volume (Figure 15e).105|106 This work focuses on the fluctuation-induced attraction between sequenced polyampholytes, but the general sequence trends are consistent with later work on high charge-density systems, such as the aforementioned transfer matrix theory from Sing and Perry (Figure 15, d) that compares favorably with experiment.138

\subsubsection{Combining Electrostatics and other Interactions}

Looking beyond the effects of charge patterning, the next steps in understanding phase separation look to combine electrostatic effects with orthogonal interactions such as hydrogen bonding, dipole-dipole interactions, $\pi$ - $\pi$ interactions, cation- $\pi$ bonding, and hydrophobic effects. However, the combination of these relatively short-range effects with Coulombic interactions results in a convergence of length scales which leads to a competition of effects that are challenging to describe both theoretically and conceptually. 160

To date, there have been only a handful of reports that have looked into the effect of combining interactions. For example, a series of reports from the Tirrell and de Pablo groups 

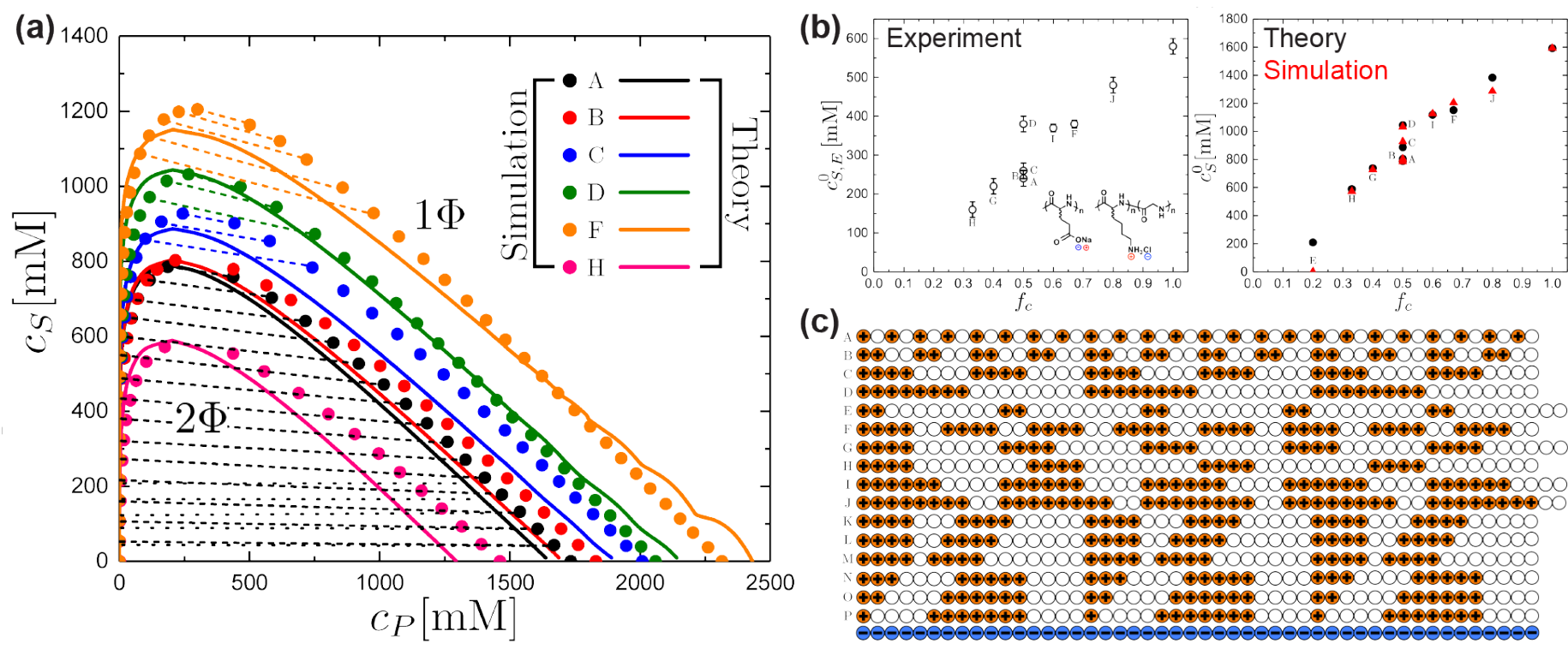

(c)

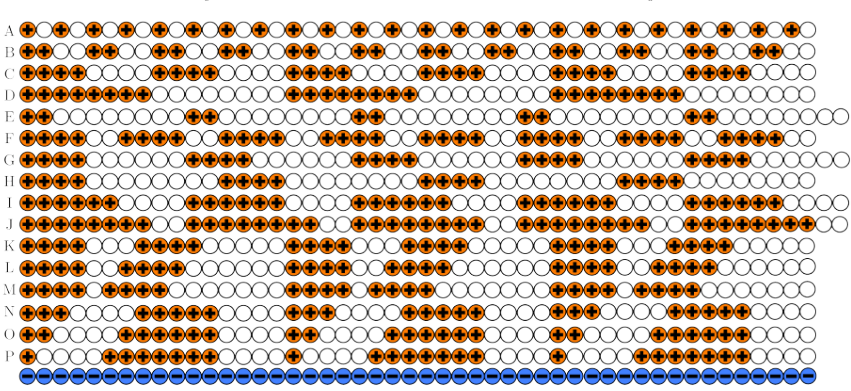

Fig. 14 Charge Patterning. (a) Coacervate phase diagram from simulation and TM theory for a number of different monomer sequences for a patterned polycation/homopolyanion system. (b) Experiment, theory, and simulation results exhibiting qualitative matching for a large number of sequences, measured by the salt resistance $\left(c_{s}^{0}\right.$, theory/simulation). (c) The entire set of sequences considered in (a) and (b). Reprinted (adapted) with permission from Ref.137, Lytle et al., ACS Central Science, 2019, 5(4), 709-718. Copyright 2019 American Chemical Society, https://pubs.acs.org/doi/10.1021/acscentsci.9b00087. Further permissions related to the material excerpted should be directed to the ACS.

described the potential for hydrogen bond formation between the backbones of oppositely-charged polypeptides during complexation. 45]140|275 A combination of experimental results using polypeptides of controlled chirality and molecular dynamics simulations demonstrated that a continuous sequence of approximately eight amino acids of the same chirality was needed to drive the formation of a stable $\beta$-sheet structure, tipping the stability of the system from liquid complex coacervates to solid precipitates. Systematic variation of hydrophobicity in both polypeptides ${ }^{139}$ and synthetic polymers $\sqrt{40|53| 64}$ has mostly demonstrated that hydrophobic polymers form denser coacervate phases, $40[53139$ though in some cases these differences were negligible. $\frac{64}{}$ In a separate study, Hyman, Alberti, and Pappu used mutagenesis studies of the FUS family of IDPs to demonstrate a hierarchy of interactions driving phase separation. 19 While electrostatic effects enhanced phase separation generally, the distribution of cation- $\pi$ interactions between arginine and phenylalanine residues affecting the material properties of the resulting coacervate in a manner consistent with the theory of associative polymers. $276 \mid 277$

\subsubsection{Conjugated Polymers}

Complex coacervates formed from conjugated polymers represent an example system where electrostatic and $\pi$ - $\pi$ stacking interactions coexist. Here, the combination of associative interactions and the potential for the exclusion of solvent from around the polymer tends to drive complexes into a solid state. Work by Danielsen et al. demonstrated the ability to form liquid coacervates through the use of cosolvent mixtures. 278 Beyond the novelty of invoking $\pi-\pi$ interactions, coacervates of conjugated polymers are particularly interesting because of their optical properties. In particular, the formation of a polyelectrolyte com- plex has been shown to result in an increased $\pi$-conjugation length, enhanced emissivity, and a dramatically increased fluorescence quantum yield. These enhanced properties have been attributed to a planar polymer architecture that leads to excited states that are highly delocalized along the polymer backbone. 279 This idea of using molecular-level interactions and self-assembly to tune materials properties is emblematic of the future potential of coacervate-based materials.

\subsection{Bioencapsulation}

While the discussion thus far has focused on complex coacervation between two relatively unstructured polyelectrolytes, the utility of complex coacervation across a range of applications has motivated the study of a wider range of macro-ions. In particular, we will highlight here studies focused on the inclusion of globular (folded) proteins.

\subsubsection{Protein-Polymer Coacervation and Theory}

Recent efforts have generated a growing body of work related to the direct complex coacervation of proteins with polymers; however, our physical understanding of the underlying interactions involved in many of these coacervating systems can be traced back to studies focused on binding interactions between proteins and DNA or RNA.

The importance of electrostatic interactions on protein/DNA binding, and the potential role of counterion release in providing an entropic driving force for this binding was identified in the late 1960s-1970s based on the observed decrease in binding constant between lac repressor and lac operator DNA with increasing salt concentration. ${ }^{280}$ This "counterion release force," identified by Record et al. ${ }^{109280}$ and further developed by Manning 281 provided a correlation between the logarithms of the binding con- 

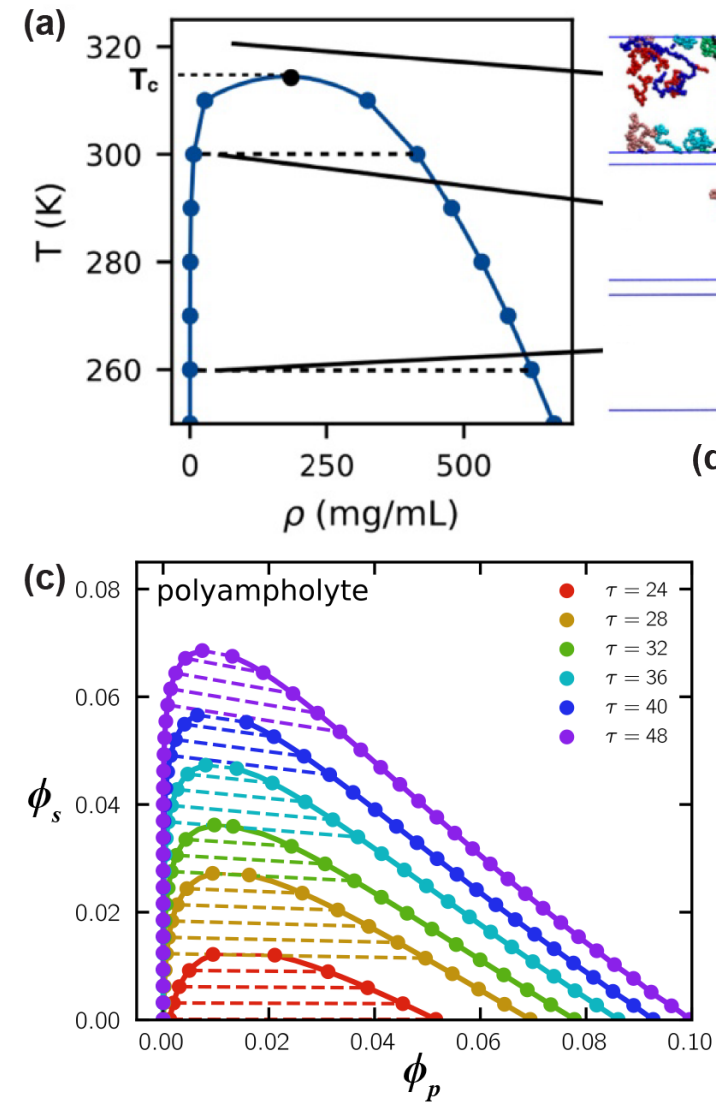

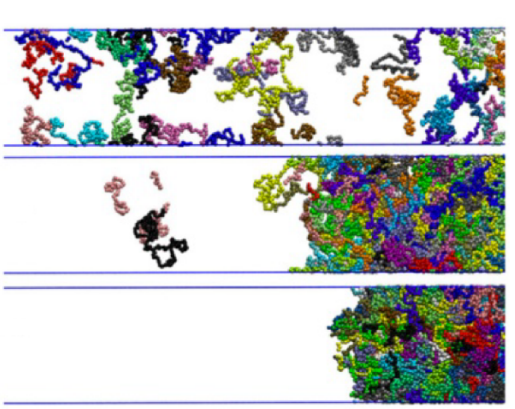

(d)
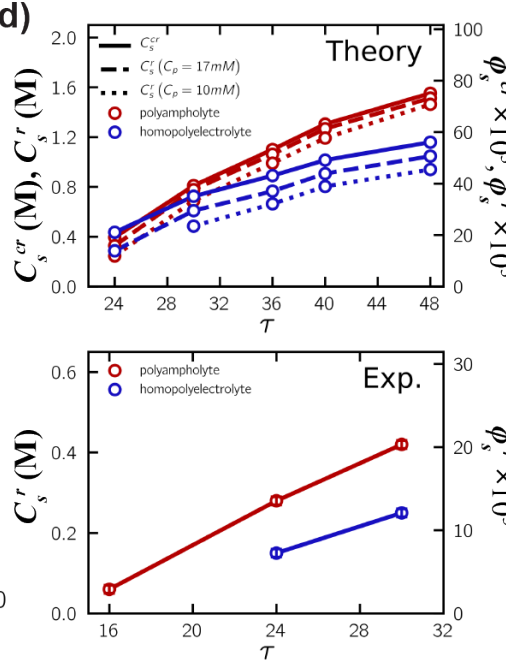

(b)

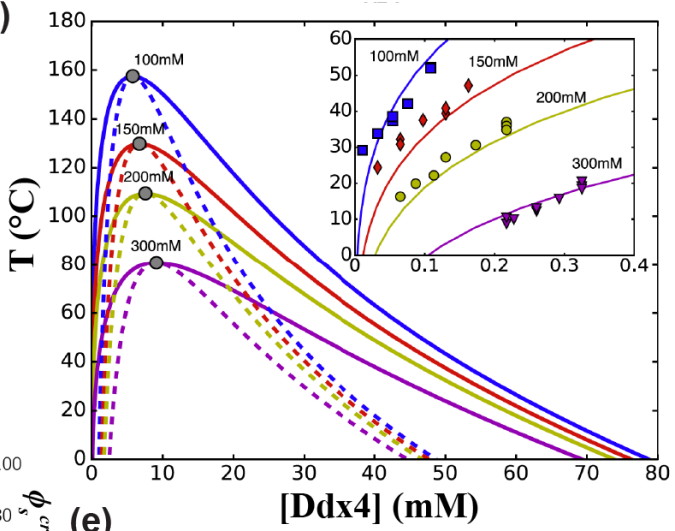

(e)

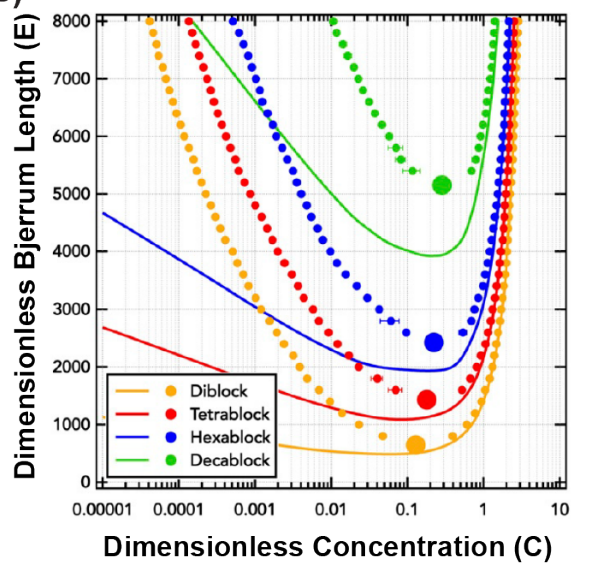

Fig. 15 Coacervate Self-Assembly. (a) Dignon et al. used the 'slab method' to calculate phase behaviors in coarse-grained models of an intrinsically disordered protein FUS, 260 treating electrostatics via screened Debye-Hückel interactions. Reproduced from Ref. ${ }^{260}$. (b) Phase diagrams for intrinsically-disordered polypeptide Ddx4, predicted from RPA and compared with experimentally determined (inset) phase boundaries. 258263 Reprinted from J. Mol. Liq., 228, Lin et al., Random-phase-approximation theory for sequence-dependent, biologically functional liquid-liquid phase separation of intrinsically disordered proteins, 176-193, Copyright 2017, with permission from Elsevier. (c) Polyampholyte phase diagrams from Madinya et al. calculated via the sequence-defined transfer matrix theory, also showing an increase in the two-phase region with increasing block length $(\tau / 2)$. 138 (d) Salt resistance $c_{S}^{r}$ and critical salt concentration $c_{s}^{c r}$ in (c) as a function of $\tau$ for both polyampholytes (red) and homopolyelectrolytes (blue), compared with experimental measurements (inset) of the same quantity from sequence-defined polypeptides. 138 (c,d) Adapted from Madinya et al. Mol. Syst. Des. Eng., 2020, Advance Article, DOI: 10.1039/C9ME00074G - Reproduced by permission of The Royal Society of Chemistry. (e) Phase diagram from Danielsen et al., as a function of $E$ (dimensionless Bjerrum length) and $C$ (dimensionless concentration) calculated for multiblock polyampholytes at constant chain lengths via field theoretic calculations. 103/104/106 Multiblocks exhibit decreasing ability to form coacervates, as block length decreases. Reproduced from Ref. 106 . 
stant and salt concentration and the number of counterions released upon binding, provided that no preferential binding or hydration interactions are present.

$$
\frac{d \ln K_{b}}{d \ln C_{\text {salt }}}=-\Delta n_{\text {ion }}
$$

This approach has been applied to a variety of systems using both ITC and fluorescence-based binding assays to provide information on the details of charge-driven binding, ${ }^{280 \mid 282}$ with experimental evidence correlating well with results from simulations. 283

While these binding studies were performed under very dilute conditions to avoid multi-body interactions, a number of studies have reported on both bulk complex coacervation 2/12 15/76/284/291 and microphase separation $\frac{73 / 292,301}{\text { involv- }}$ ing globular proteins and polyelectrolytes. Of particular note from these studies is the identification of a critical charge content necessary for complex coacervation to occur between a protein and an oppositely-charged polymer. 286 Additionally, a higher charge content was required for the formation of stable coacervate-core micelles because of the additional conformational constraints imposed by the presence of the micellar interface.

Recently, fundamental numerical theory has provided an alternative picture to the counterion release mechanisms of Record, et al. 280 and Manning, 1et al.281 to understand polymer-particle and polymer-protein coacervation. 302 These efforts have used polymer self-consistent field theory to determine pairwise and three-body interaction potentials between charged particles. These efforts were used to predict phase boundaries for these asymmetric systems, which not only consider the electrostatic interactions but other competing effects such as polymer depletion and colloidal radius. 303 Finally, extensions to particles with oppositely-charged patches provides insights into proteinpolymer coacervation. 302

\subsubsection{Designer Sequences for Selective Encapsulation}

In addition to net charge, the clustering of charge can have a significant impact on coacervation. For example, the localization of charge onto a terminal tag resulted in a much stronger tendency for variants of green fluorescent protein (GFP) to coacervate, as compared with variants where the same net charge was distributed isotropically across the protein surface (Figure 16, 15 Similarly, the presence of a cluster of charges on the protein lysozyme resulted in significantly higher levels of protein incorporation into a two-polymer coacervate than for a serum protein where the surface charges were isotropically distributed.291 These examples highlight open questions and opportunities in the field for using charge specificity to facilitate uptake, separation, release, etc.

\subsection{New Directions for Coacervate Modeling and Theory}

Recent progress has been due to a confluence of both experimental and theoretical/computational investigations. The central role played by modeling and theory is enabling to the emerging directions in coacervation, especially as the phenomena of coacervation is applied to an increasing list of polymer chemistries, ar-

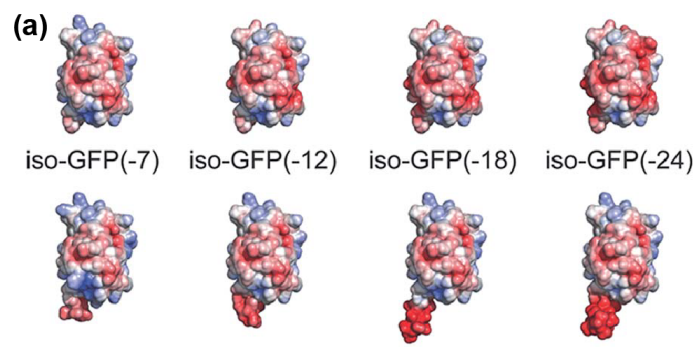

tag-GFP(-7) tag-GFP(-12) tag-GFP(-18) tag-GFP(-24)
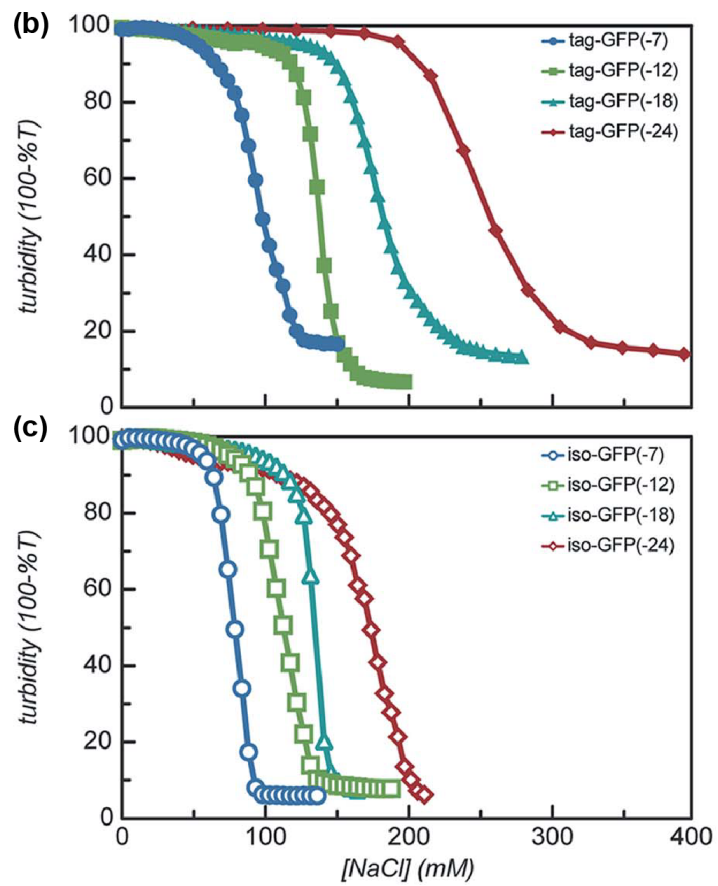

Fig. 16 Bioencapsulation. (a) Electrostatic surface potential representations of the solvent accessible surface area for GFP variants as calculated using the linearized Poisson-Boltzmann equation. Positive residues are colored blue, negative residues are red. Titration studies showing the effect of salt on complexes of (b) ionically-tagged GFP and (c) isotropic charge varients of GFP in complex with quaternized poly(4-vinyl N-methyl pyridinium iodide) in $10 \mathrm{mM}$ Tris buffer, $\mathrm{pH}$ 7.4. Figure adapted from Ref. 15, Kapelner and Obermeyer, Chem. Sci., 2019, 9(4), e1442 - Published by The Royal Society of Chemistry. 
chitectures, and applications, including proteins. The community has begun to establish the structure-property relationships in this field, but there remain a number of key areas where advances in theory and simulation will be important.

Atomistic detail has, except for a few studies, $167|200| 203|206| 207$ been largely neglected; yet, variations in polymer chemistry and salt identity lead to significant differences in coacervate phenomena.40/45/53/139 It is unclear the extent to which coarse-grained models will be able to - even with careful parameterization - capture the physical aspects of these differences. What is more apparent, however, is that there are at least some situations where an atomistic view is crucial. One area is broadly related to the presence of non-equilibrium complexes, often referred to as 'precipitates' that do not form the liquid coacervate droplets/phases that prior theory predicts. This is where there is already some atomistic simulation work, with a focus on polypeptide chirality 188275 and glassy polyelectrolyte complexes has made progress.167200|203/206/207 These studies have elucidated key aspects of these non-equilibrium states, including the central role of water structure in complex dynamics $167|200| 203 / 205 / 207$ and the importance of racemic peptide sequences in the formation of polypeptide coacervates. $\frac{1882275}{2}$ However, these specific dynamic and structural effects represent only initial forays into understanding these complexes; for example, there is no comprehensive understanding of how and why these trapped states occur, nor is there any consensus on the highly process-dependent molecular structure. This may stymie the application of polymer complex coacervates, as the kinetically-trapped precipitate is typically undesirable in the formation of predictable materials.

Another area where atomistic simulation will be needed is in the elucidating the role of water in coacervate interactions (both electrostatic and non-electrostatic). This is a challenging field of physical chemistry, and even simple salts are notoriously difficult to understand; it is accepted that there are no coarse-grained parameterizations agnostic to all physical circumstances. ${ }^{306}$ There are also non-trivial effects on non-electrostatic interactions, with evidence that e.g., monomer sequence can alter water structure in a way that has significant effects on hydrophobic interactions. 307308

Atomistic effects are thus extremely important, yet it is a major modeling challenge to resolve these details in the context of the prior work that uses coarse-grained representations. There is a need to incorporate short time/length scales into the larger-scale phase behaviors studied in most of the existing coacervate literature, and this will require new advances in multiscale modeling beyond the current state of the art.

Coacervate dynamics represent a related area of need, in part due to the challenge of modeling kinetically-trapped complexes.4750|188 However, even for coacervates that are visibly liquid, theory and modeling has not extensively studied dynamics. Much of the current theory mostly revolves around the incorporation of Debye-Hückel arguments into a sticky-Rouse model. $375760 \mid 309$ While this provides some insight, this picture is inconsistent with the recent developments outlined in this review showing that Debye-Hückel is insufficient to describe coacervate phase behavior. ${ }^{33|35| 87}$ Yet, there are theoretical and com- putational challenges that pose a challenge to progress in coacervate dynamics. For simulation, limitations for coacervates are the same as the limitations for any non-dilute polymer system, except exacerbated by the need to model counterions and electrostatic interactions. In particular, polymer solution dynamics require consideration of hydrodynamic interactions that represent the coupled motions of charges due to local solvent flows.117310 314 These are extremely computationally expensive, especially for polymers in the so-called 'semidilute' regime that extists above the 'overlap' concentration that is typical of coacervates; here, the polymer solution is characterized by extensive interpolymer interactions as polymer conformations begin to impinge upon each other. ${ }^{118}$ There have been very few examples of simulations of semidilute polymer dynamics, with most focusing on algorithmic advances to circumvent the otherwise-prohibitive computational cost of performing these calculations. ${ }^{315}[19$ Thus, it remains unclear how feasible it is to perform molecular simulations of coacervates, with sufficient molecular resolution to see how e.g., small molecule ions may affect the dynamics observed in experiment. 3201321 The detailed motion of coacervate chains and ions represents a challenge, but also an opportunity to clarify the molecular interpretation of salt effects in coacervate dynamics. 37,5760

Non-polymer/polymer coacervation is of primary relevance to the application of polymers in both industry as well as biological systems, where coacervate-forming species include surfactant micelles, folded proteins, and nanoparticles. 12 14476/284|285|287/289|296/305/322/327| Here, molecular geometry and surface-charge patterning will play a significant role in the prediction of coacervate properties; it will no longer be possible to take advantage of (i) the symmetry between the larger charged species and (ii) the highly interpenetrating conformations, both of which help the theoretical development of polyelectrolytepolyelectrolyte systems. Only a few theoretical or computational papers have considered these systems,, $302 / 303 / 328 / 330$ with the bulk of the work performed experimentally. Much of the insight developed in recent advances in coacervate theory could be modified to account for these more complicated scenarios; however, many of the methods may need to be combined in new ways. For example, the spatial structure of polymer/colloid interactions would require theoretical tools such as polymer field theory or PRISM theory. $172[302[331+333$ The theoretical challenge is to include models or simulations that capture local charge correlations into these larger length-scale methods, especially in the case of high charge-density polymers. 31 87/97/177

\section{Outlook and Conclusions}

Recent advances in the science and engineering of complex coacervates has provided the foundation for their practical use in a variety of applications, ranging from self-assembled, functional materials to tunable material encapsulants and industrially-practical viscosity modifiers. This foundation is now built on a rich array of theory and simulation tools that provide physical insights into materials design, which is crucial to navigate the extensive parameter space spanned by even simple complex coacervate systems. If recent efforts are any indication, coacervation not only has in- 
teresting problems that remain to be addressed, but the study of these problems will provide insight to a large number of peripheral areas. Connections to sequence-defined polymers, glassy dynamics, and intrinsically-disordered protein biophysics are already apparent - and areas of active inquiry that we have mentioned. Looking forward, we anticipate that the need to reckon with the role of hydration and atomistic detail, the possibility of higher-order molecular self-assembly with sequence-defined polyelectrolytes, and the importance of non-electrostatic interactions in biology are all emerging areas in both coacervation as well starting points for deeply challenging questions in molecular engineering and chemical physics. Thus, despite the extensive efforts of the community to date, there remain endless opportunities for polymer scientists - both experimentalists and theorists - to find interesting problems the study of complex coacervation.

\section{Conflicts of interest}

There are no conflicts to declare.

\section{Acknowledgements}

C.E.S. acknowledges support from the National Science Foundation under NSF CAREER Award No. DMR-1654158. S.L.P. acknowledges support from the National Science Foundation under NSF CBET-1804177 and CMMI-1727660.

\section{Notes and references}

1 M. Muthukumar, Macromolecules, 2017, 50, 9528-9560.

2 J. v. d. Gucht, E. Spruijt, M. Lemmers and M. A. Cohen Stuart, J. Colloid Interface Sci., 2011, 361, 407-422.

3 S. Srivastava and M. V. Tirrell, Advances in Chemical Physics, John Wiley and Sons, Hoboken, NJ, 2016.

4 S. L. Perry, Curr. Opin. Colloid Interface Sci., 2019, 39, 86-97.

5 S. L. Turgeon, C. Schmitt and C. Sanchez, Curr. Opin. Colloid Interface Sci., 2007, 12, 166-178.

6 C. Schmitt and S. L. Turgeon, Adv. Colloid Interface Sci., 2011, 167, 63-70.

7 A. Matalanis, O. G. Jones and D. J. McClements, Structured biopolymer-based delivery systems for encapsulation, protection, and release of lipophilic compounds, 2011, 25, 18651880.

8 H. G. B. d. Jong and H. R. Kruyt, Proc. Koninklijke Nederlandse Akademie Wetenschappen, 1929, 32, 849-856.

9 J. T. G. Overbeek and M. Voorn, J. Cell. Comp. Physiol., 1957, 49, 7-26.

10 F. Tiebackx, Zeitschrift für Chemie und Industrie der Kolloide, 1911, 1, 198-201.

11 P. Welch and M. Muthukumar, Macromolecules, 2000, 33, 6159-6167.

12 C. L. Cooper, P. L. Dubin, A. B. Kayitmazer and S. Turksen, Curr. Opin. Colloid Interface Sci., 2005, 10, 52-78.

13 C. G. d. Kruif, F. Weinbreck and R. d. Vries, Curr. Opin. Colloid Interface Sci., 2004, 9, 340-349.

14 E. Kizilay, A. B. Kayitmazer and P. L. Dubin, Adv. Colloid Interface Sci., 2011, 167, 24-37.
15 R. A. Kapelner and A. C. Obermeyer, Chem. Sci., 2019, 9, e1442.

16 J. E. Laaser, M. McGovern, Y. Jiang, E. Lohmann, T. M. Reineke, D. C. Morse, K. D. Dorfman and T. P. Lodge, J. Phys. Chem. B, 2017, 121, 4631-4641.

17 P. J. Flory, Principles of Polymer Chemistry, Cornell University Press, Ithaca, NY, 1953.

18 C. P. Brangwynne, P. Tompa and R. V. Pappu, Nat. Phys., 2015, 11, 899-904.

19 J. Wang, J.-M. Choi, A. S. Holehouse, H. O. Lee, X. Zhang, M. Jahnel, S. Maharana, R. Lemaitre, A. Pozniakovsky, D. Dreschel, I. Poser, R. V. Pappu, S. Alberti and A. A. Hyman, Cell, 2018, 174, 688-699.

20 T. J. Nott, E. Petsalaki, P. Farber, D. Jervis, E. Fussner, A. Plochowietz, T. D. Craggs, D. P. Bazett-Jones, T. Pawson, J. D. Forman-Kay and A. J. Baldwin, Mol. Cell, 2015, 57, 936947.

21 E. W. Martin and T. Mittag, Biochemistry, 2018, 57, 24782487.

22 C. W. Pak, M. Kosno, A. S. Holehouse, S. B. Padrick, A. Mittal, R. Ali, A. A. Yunus, D. R. Liu, R. V. Pappu and M. K. Rosen, Mol. Cell, 2016, 63, 72-85.

23 I. Michaeli, J. T. G. Overbeek and M. Voorn, J. Polym. Sci., 1957, 23, 443-450.

24 P. Debye and E. Huckel, Phys. Z., 1923, 24, 185.

25 D. A. McQuarrie, Statistical Mechanics, University Science Books, Sausalito, 2000.

26 K. I. Tainaka, Biopolymers, 1980, 19, 1289-1298.

27 E. Spruijt, A. H. Westphal, J. W. Borst, M. A. Cohen Stuart and J. van der Gucht, Macromolecules, 2010, 43, 6476-6484.

28 P. K. Jha, P. S. Desai, J. Li and R. G. Larson, Polymers, 2014, 6, 1414-1436.

29 R. Zhang and B. I. Shklovskii, Physica A, 2005, 352, 216238.

30 P. Zhang, N. M. Alsaifi, J. Wu and Z.-G. Wang, J. Chem. Phys., 2018, 149, 163303.

31 T. K. Lytle and C. E. Sing, Soft Matter, 2017, 13, 7001-7012.

32 D. Priftis, X. Xia, K. O. Margossian, S. L. Perry, L. Leon, J. Qin, J. J. d. Pablo and M. Tirrell, Macromolecules, 2014, 47, 3076-3085.

33 C. E. Sing, Adv. Colloid Interface Sci., 2017, 239, 2-16.

34 M. Radhakrishna, K. Basu, Y. Liu, R. Shamsi, S. L. Perry and C. E. Sing, Macromolecules, 2017, 50, 3030-3037.

35 L. Li, S. Srivastava, M. Andreev, A. B. Marciel, J. J. de Pablo and M. V. Tirrell, Macromolecules, 2018, 51, 2988-2995.

36 P. Zhang, K. Shen, N. M. Alsaifi and Z.-G. Wang, Macromolecules, 2018, 51, 5586-5593.

37 E. Spruijt, J. Sprakel, M. A. Leermakers, F. A. M. Cohen Stuart and J. v. d. Gucht, Phys. Rev. Lett., 2010, 105, 208301.

38 E. Spruijt, J. Sprakel, M. A. Cohen Stuart and J. v. d. Gucht, Soft Matter, 2010, 6, 172-178.

39 J. Qin, D. Priftis, R. Farina, S. L. Perry, L. Leon, J. Whitmer, K. Hoffmann, M. Tirrell and J. J. d. Pablo, ACS Macro Letters, 2014, 6, 565-568. 
40 J. Lou, S. Friedowitz, J. Qin and Y. Xia, ACS Cent. Sci., 2019, 5, 549-557.

41 R. Chollakup, W. Smitthipong, C. Eisenback and M. Tirrell, Macromolecules, 2010, 43, 2518-2528.

42 R. Chollakup, J. B. Beck, K. Dirnberger, M. Tirrell and C. D. Eisenbach, Macromolecules, 2013, 46, 2376-2390.

43 D. Priftis, N. Laugel and M. Tirrell, Langmuir, 2012, 28, 15947-15957.

44 D. Priftis and M. Tirrell, Soft Matter, 2012, 8, 9396-9405.

45 S. L. Perry, Y. Li, D. Priftis, L. Leon and M. Tirrell, Polymers, 2014, 6, 1756-1772.

46 B. M. Johnston, C. W. Johnston, R. A. Letteri, T. K. Lytle, C. E. Sing, T. Emrick and S. L. Perry, Org. Biomol. Chem., 2017, 15, 7630-7642.

47 Y. Liu, M. B., H. Winter and S. Perry, Soft Matter, 2017, 13, 7332-7340.

48 S. Ali, B. M. and V. Prabhu, ACS Macro Letters, 2019, 8, 289293.

49 M. T. Wei, S. Elbaum-Garfinkle, A. S. Holehouse, C. C. H. Chen, M. Feric, C. B. Arnold, R. D. Priestley, R. V. Pappu and C. P. Brangwynne, Nat. Chem., 2017, 9, 1118-1125.

50 Q. Wang and J. B. Schlenoff, Macromolecules, 2014, 47, 3108-3116.

51 J. Fu and J. B. Schlenoff, J. Am. Chem. Soc., 2016, 138, 980990.

52 J. Fu, F. H.M. and J. B. Schlenoff, Macromolecules, 2017, 50, 1066-1074.

53 K. Sadman, Q. Wang, Y. Chen, B. Keshavarz, Z. Jiang and K. Shull, Macromolecules, 2017, 50, 9417-9426.

54 S. Lim, D. Moon, H. J. Kim, S. H. Seo, I. S. Kang and H. J. Cha, Langmuir, 2014, 30, 1108-1115.

55 D. Priftis, R. Farina and M. Tirrell, Langmuir, 2012, 28, 8721-8729.

56 D. S. Hwang, H. Zeng, A. Srivastava, D. V. Krogstad, M. Tirrell, J. N. Israelachvili and J. Waite, Soft Matter, 2010, 6, 3232-3236.

57 E. Spruijt, M. A. Cohen Stuart and J. v. d. Gucht, Macromolecules, 2013, 46, 1633-1641.

58 Y. Liu, H. H. Winger and S. L. Perry, Adv. Colloid Interface Sci., 2017, DOI:10.1016/j.cis.2016.08.010.

59 S. Ali and V. Prabhu, Gels, 2018, 4, 11.

60 F. Hamad, Q. Chen and R. Colby, Macromolecules, 2018, 51, 5547-5555.

61 M. Tekaat, D. Bütergerds, M. Schönhoff, A. Fery and C. Cramer, Phys. Chem. Chem. Phys., 2015, 17, 2255222556.

62 A. B. Marciel, S. Srivastava and M. V. Tirrell, Soft Matter, 2018, DOI: 10.1039/C7SM02041D.

63 P. Suarez-Martinez, P. Batys, M. Sammalkorpi and J. Lutkenhaus, Macromolecules, 2019, 52, 3066-3074.

64 J. Huang, F. Morin and J. E. Laaser, Macromolecules, 2019, 52, 4957-4967.

65 J. Sun, S. Perry and J. Schiffman, Biomacromolecules, 2019, 20, 4191-4198.
66 E. Spruijt, F. A. M. Leermakers, R. Fokkink, R. Schweins, A. A. v. Well, M. A. Cohen Stuart and J. van der Gucht, Macromolecules, 2013, 46, 4596-4605.

67 M. Z. Markarian, H. H. Hariri, A. Reisch, V. S. Urban and J. B. Schlenoff, Macromolecules, 2012, 45, 1016-1024.

68 X. Feng, M. Leduc and R. Pelton, Colloids Surf., A, 2008, 317, 535-542.

69 D. Priftis, K. Megley, N. Laugel and M. Tirrell, J. Coll. Interf. Sci., 2013, 398, 39-50.

70 L. W. Chang, T. K. Lytle, M. Radhakrishna, J. J. Madinya, J. VÂllez, C. E. Sing and S. L. Perry, Nat. Commun., 2017, 8, 1273.

71 A. B. Kayitmazer, Adv. Colloid Interface Sci., 2017, 239, 169177.

72 L. Vitorazi, N. Ould-Moussa, S. Sekar, J. Fresnais, W. Woh, J.-P. Chapel and J.-F. Berret, Soft Matter, 2014, 10, 94969505.

73 I. K. Voets, A. de Keizer and M. A. Cohen Stuart, Adv. Colloid Interface Sci., 2009, 147, 300-318.

74 M. A. Cohen Stuart, N. A. M. Besseling and R. G. Fokkink, Langmuir, 1998, 14, 6846-6849.

75 A. Harada and K. Kataoka, Macromolecules, 1995, 28, 5294 5299.

76 K. A. Black, D. Priftis, S. L. Perry, J. Yip, W. Y. Byun and M. Tirrell, ACS Macro Lett., 2014, 3, 1088-1091.

77 A. Harada and K. Kataoka, Science, 1999, 283, 65-67.

78 N. Johnson and Y. Wang, Expert Opin. Drug Discovery, 2014, 11, 1829-1832.

79 R. J. Stewart, C. S. Wang and H. Shao, Adv. Colloid Interface Sci., 2011, 167, 85-93.

80 A. B.K., S. Das, R. Linstadt, Y. Kaufman, N. MartinezRodriguez, R. Mirshafian, E. Kesselman, Y. Talmon, B. Lipshutz, J. N. Israelachvili and J. Waite, Nat. Commun., 2015, 6, 8663.

81 R. J. Stewart, C. S. Wang, I. Song and J. Jones, Adv. Colloid Interface Sci., 2017, 239, 88-96.

82 D. J. Audus, J. D. Gopez, D. V. Krogstad, N. A. Lynd, E. J. Kramer, C. J. Hawker and G. H. Fredrickson, Soft Matter, 2015, 11, 1214-1225.

83 S. Srivastava, M. Andreev, A. E. Levi, D. J. Goldfeld, J. Mao, W. T. Heller, J. J. de Pablo and M. V. Tirrell, Nat. Commun., 2017, 8, 14131.

84 D. V. Krogstad, N. A. Lynd, S.-H. Choi, J. M. Spruell, C. J. Hawker, E. J. Kramer and M. V. Tirrell, Macromolecules, 2013, 46, 1512-1518.

85 D. V. Krogstad, S.-H. Choi, N. A. Lynd, D. J. Audus, S. L. Perry, J. D. Gopez, C. J. Hawker, E. J. Kramer and M. V. Tirrell, J Phys. Chem., 2014, 118, 13011-13018.

86 D. V. Krogstad, N. A. Lynd, D. Miyajima, J. Gopez, C. J. Hawker, E. J. Kramer and M. V. Tirrell, Macromolecules, 2014, 47, 8026-8032.

87 S. L. Perry and C. E. Sing, Macromolecules, 2015, 48, 50405053.

88 R. A. Riggleman, R. Kumar and G. H. Fredrickson, J. Chem. 
Phys., 2012, 136, 024903.

89 X. Du, D. Seeman, P. L. Dubin and D. Hoagland, Langmuir, 2015, 31, 8661-8666.

90 I. Nakamura, N. P. Balsara and Z.-G. Wang, Phys. Rev. Lett., 2011, 107, 198301.

91 I. Nakamura, Soft Matter, 2014, 10, 9596-9600.

92 X. Duan and I. Nakamura, Soft Matter, 2015, 11, 35663571.

93 A. Levy, D. Andelman and H. Orland, Phys. Rev. Lett., 2012, 108, 227801.

94 R. Kumar, B. G. Sumpter and M. Muthukumar, Macromolecules, 2014, 47, 6491-6502.

95 Y.-Z. Wei, P. Chiang and S. Sridhar, J. Chem. Phys., 1992, 96, 4569-4573.

96 R. Kumar and G. H. Fredrickson, J. Chem. Phys., 2009, 131, 104901.

97 A. Salehi and R. G. Larson, Macromolecules, 2016, 49, 97069719.

98 M. Radhakrishna and C. E. Sing, Macromol. Chem. Phys., 2016, 217, 126-136.

99 A. Kudlay, A. V. Ermoshkin and M. O. d. 1. Cruz, Macromolecules, 2004, 37, 9213-9241.

100 A. Kudlay and M. Olvera de la Cruz, J. Chem. Phys., 2004, 120, 404-412.

101 E. Sokolova, E. Spruijt, M. M. K. Hansen, E. Dubuc, J. Groen, V. Chokkalingam, A. P. H. A. Heus and W. T. S. Huck, Proc. Natl. Acad. Sci. U. S. A., 2013, 110, 11692-11697.

102 G. Fredrickson, The Equilibrium Theory of Inhomogeneous Polymers, Oxford University Press, 2006.

103 J. Lee, Y. O. Popov and G. H. Fredrickson, J. Chem. Phys., 2008, 128, 224908.

104 K. T. Delaney and G. H. Fredrickson, J. Chem. Phys., 2017, 146, 224902.

105 S. P. O. Danielsen, J. McCarty, J.-E. Shea, K. Delaney and G. Fredrickson, J. Chem. Phys., 2019, 151, 034904.

106 S. P. O. Danielsen, J. McCarty, J.-E. Shea, K. T. Delaney and G. H. Fredrickson, Proc. Natl. Acad. Sci. U. S. A., 2019, 82248232.

107 Z. Wang and M. Rubinstein, Macromolecules, 2006, 39, 5897-5912.

108 N. Shusharina, E. Zhulina, A. Dobrynin and M. Rubinstein, Macromolecules, 2005, 38, 8870-8881.

109 M. T. Record, C. F. Anderson and T. M. Lohman, Q. Rev. Biophys., 1978, 11, 103-178.

110 G. S. Manning, J. Chem. Phys., 1969, 51, 924-933.

111 S. Liu and M. Muthukumar, J. Chem. Phys., 2002, 116, 9975.

112 J. Qin and J. J. de Pablo, Macromolecules, 2016, 49, 87898800 .

113 A.-C. Shi and J. Noolandi, Macromol. Theory Simul., 1999, 8, 214-229.

114 Q. Wang, T. Taniguchi and G. H. Fredrickson, J. Phys. Chem. $B, 2004,108,6733-6744$.

115 V. Y. Borue and I. Y. Erukhimovich, Macromolecules, 1990, 23, 3625-3632.
116 M. Castelnovo and J.-F. Joanny, Eur. Phys. J. E: Soft Matter Biol. Phys., 2001, 6, 377-386.

117 d. P.G., Scaling concepts in polymer physics, Cornell University Press, 1979.

118 R. M. and R. Colby, Polymer physics, Oxford University Press, 2003.

119 M. Rubinstein, Q. Liao and S. Panyukov, Macromolecules, 2018, 51, 9572-9588.

120 A. M. Rumyantsev, E. B. Zhulina and O. V. Borisov, Macromolecules, 2018, 51, 3788-3801.

121 A. M. Rumyantsev and J. J. d. Pablo, Macromolecules, 2019, 52, 5140-5156.

122 A. Dobrynin, C. R.H. and M. Rubinstein, Macromolecules, 1995, 28, 1859-1871.

123 A. M. Rumyantsev, E. B. Zhulina and O. V. Borisov, ACS Macro Lett., 2018, 7, 811-816.

124 R. Nyquist, B.-Y. Ha and A. Liu, Macromolecules, 1999, 32, 3481-3487.

125 P. Gonzalez-Mozuelos and M. Olvera de la Cruz, J. Chem. Phys., 1995, 103, 3145-3157.

126 A. Dobrynin and M. Rubinstein, Prog. Polym. Sci., 2005, 30, 1049-1118.

127 R. Netz and H. Orland, Eur. Phys. J. E, 2003, 11, 301-311.

128 M. Stevens, Biophys. J., 2001, 80, 130-139.

129 Q. Liao, A. Dobrynin and M. Rubinstein, Macromolecules, 2003, 36, 3399-3410.

130 M. Deserno, C. Holm and S. May, Macromolecules, 2000, 33, 199-206.

131 Z. Ou and M. Muthukumar, J. Chem. Phys., 2006, 124, 154902.

132 B. Peng and M. Muthukumar, J. Chem. Phys., 2015, 143, 243133.

133 R. M. Elder, T. Emrick and A. Jayaraman, Biomacromolecules, 2011, 12, 3870-3879.

134 X. Xu, M. Kanduc, J. Wu and J. Dzubiella, J. Chem. Phys., 2016, 145, 034901.

135 S. Adhikari, M. A. Leaf and M. Muthukumar, J. Chem. Phys., 2018, 149, 163308.

136 D. Priftis, L. Leon, Z. Song, S. Perry, K. Margossian, A. Tropnikova, J. Cheng and M. Tirrell, Angew. Chem. Int. Ed., 2015, 54, 11128-11132.

137 T. K. Lytle, L.-W. Chang, N. Markiewicz, S. L. Perry and C. E. Sing, ACS Cent. Sci., 2019, 5, 709-718.

138 J. J. Madinya, L.-W. Chang, S. L. Perry and C. E. Sing, Mol. Syst. Des. Eng., 2019, DOI:10.1039/C9ME00074G.

139 S. Tabandeh and L. Leon, Molecules, 2019, 24, 868.

140 N. M. Pacalin, L. Leon and M. Tirrell, Eur. Phys. J. Special Topics, 2016, 225, 1805-1815.

141 J. F. Lutz, M. Ouchi, D. R. Liu and M. Sawamoto, Science, 2013, 341, 1238149.

142 J. F. Lutz, J. M. Lehn, E. W. Meijer and K. Matyjaszewski, Nat. Rev. Mater., 2016, 1, 1-14.

143 M. G. T. A. Rutten, F. W. Vaandrager, J. A. A. W. Elemans 
and R. J. M. Nolte, Nat. Rev. Chem., 2018, 2, 365-381.

144 S. Parelkar, R. Letteri, D. Chan-Seng, O. Zolochevska, J. Ellis, M. Figueiredo and T. Emrick, Biomacromolecules, 2014, 15, 1328 âĂŞ-1336.

145 Y. Zhang and P. Cremer, Curr. Opin. Chem. Biol., 2006, 10, 658-663.

146 K. Collins, Biophys. J., 1997, 72, 65-76.

147 K. Collins, Methods, 2004, 34, 300-311.

148 K. Collins, Biophys. Chem., 2006, 119, 271-281.

149 N. Vinh, S. Allen and K. Plaxco, J. Am. Chem. Soc., 2011, 133, 8942-8947.

150 D. George, A. Charkhesht and N. Vinh, Rev. Sci. Instrum., 2015, 86, 123105.

151 V. S. Rathee, H. Sidky, B. J. Sikora and J. K. Whitmer, J. Am. Chem. Soc., 2018, 140, 15319-15328.

152 M. Muthukumar, J. Chem. Phys., 2004, 120, 9343-9350.

153 J. P. Hansen and I. R. McDonald, Theory of Simple Liquids, Elsevier, Boston, 2006.

154 S. Friedowitz, A. Salehi, R. G. Larson and J. Qin, J. Chem. Phys., 2018, 149, 163335.

155 A. Yethiraj and C. Y. Shew, Phys. Rev. Lett., 1996, 77, 39373940.

156 A. Yethiraj, J. Chem. Phys., 1998, 108, 1184.

157 A. Yethiraj, J. Phys. Chem. B, 2008, 113, 1539-1551.

158 C. E. Sing, J. W. Zwanikken and M. O. d. 1. Cruz, Macromolecules, 2013, 46, 5053-5065.

159 C. E. Sing, J. W. Zwanikken and M. O. d. 1. Cruz, Nat. Mater., 2014, 13, 694-698.

160 C. E. Sing, J. W. Zwanikken and M. O. d. 1. Cruz, J. Chem. Phys., 2015, 142, 034902.

161 J. Brown, Y. Seo and L. Hall, Phys. Rev. Lett., 2018, 120, 127801.

162 J. Brown, Y. Seo, T. Maula and L. Hall, J. Chem. Phys., 2016, 144, 124904.

163 K. S. Schweizer and J. G. Curro, Phys. Rev. Lett., 1987, 58, 246-249.

164 A. Yethiraj and K. Schweizer, J. Chem. Phys., 1992, 97, 14551464.

165 J. W. Zwanikken, P. K. Jha and M. O. d. 1. Cruz, J. Chem. Phys., 2011, 135, 064106.

166 P. Zhang, N. M. Alsaifi, J. Wu and Z.-G. Wang, Macromolecules, 2016, 49, 9720-9730.

167 R. Zhang, Y. Zhang, H. S. Antila, J. L. Lutkenhaus and M. Sammalkorpi, J. Phys. Chem. B, 2017, 121, 322-333.

168 T. Boublik, J. Chem. Phys., 1970, 53, 471-472.

169 G. Mansoori, N. Carnahan, K. Starling and T. Leland Jr., J. Chem. Phys., 1971, 54, 1523-1525.

170 J. Jiang, L. Blum, O. Bernard and J. Prausnitz, Mol. Phys., 2001, 99, 1121-1128.

171 M. Wertheim, J. Chem. Phys., 1987, 87, 7323-7331.

172 T. Martin, T. Gartner III, R. Jones, C. Snyder and A. Jayaraman, Macromolecules, 2018, 51, 2906-2922.

173 M. Olvera de la Cruz, L. Belloni, M. Delsanti, J. Dalbiez,
O. Spalla and M. Drifford, J. Chem. Phys., 1995, 103, 57815791.

174 E. Raspaud, M. Olvera de la Cruz, J. Sikorav and F. Livolant, Biophys. J., 1998, 74, 381-393.

175 S. Adhikari, V. Prabhu and M. Muthukumar, Macromolecules, 2019, 52, 6998-7004.

176 T. K. Lytle and C. E. Sing, Mol. Syst. Des. Eng., 2018, 3, 183196.

177 T. K. Lytle, A. J. Salazar and C. E. Sing, J. Chem. Phys., 2018, 149, 163315.

178 R. Breitenkamp and T. Emrick, Biomacromolecules, 2008, 9, 2495-2500.

179 T. K. Lytle, M. Radhakrishna and C. E. Sing, Macromolecules, 2016, 49, 9693-9705.

180 P. Hagerman, Annu. Rev. Biophys. Biophys. Chem., 1988, 17, 265-286.

181 J. Perez-Vilar and R. Hill, J. Biol. Chem., 1999, 274, 3175131754.

182 L. Kjellen and U. Lindahl, Annu. Rev. Biochem., 1991, 60, 443-475.

183 J. Peter-Katalinic, Meth. Enzymol., 2005, 405, 139-171.

184 J. R. Vieregg, M. Lueckheide, M. A. B, L. Leon, B. A. J, R. J. R and M. V. Tirrell, J. Am. Chem. Soc., 2018, 140, 1632-1638.

185 R. M. Elder and A. Jayaraman, Macromolecules, 2012, 45, 8083-8096.

186 R. M. Elder and A. J. Jayaraman, J. Phys. Chem. B, 2013, 117, 11988-11999.

187 A. Shakya, M. Girard, J. King and M. Olvera de la Cruz, Macromolecules, 2020, 53, DOI: 10.1021/acs.macromol.9b02355.

188 S. L. Perry, L. Leon, K. Q. Hoffmann, M. J. Kade, D. Priftis, K. A. Black, D. Wong, R. A. Klein, C. F. P. III, K. O. Margossian, J. K. Whitmer, J. Qin, J. J. d. Pablo and M. Tirrell, Nat. Commun., 2015, 6, 6052.

189 A. Michaels, Ind. Eng. Chem., 1965, 57, 32-40.

190 C. Porcel and J. B. Schlenoff, Biomacromolecules, 2009, 10, 2968-2975.

191 H. Hariri and J. B. Schlenoff, Macromolecules, 2010, 43, 8656-8663.

192 P. Schaaf and J. B. Schlenoff, Adv. Mater., 2015, 27, 24202432.

193 T. Phoeung, M. Spanedda, E. Roger, B. Heurtault, S. Fournel, A. Reisch, A. Mutschler, F. Perrin-Schmitt, J. Hemmerlé, D. Collin, M. Rawiso, F. Boulmedais, P. Schaaf, P. Lavalle and B. Frisch, Chem. Mater., 2017, 29, 10418-10425.

194 R. F. Shamoun, A. Reisch and J. B. Schlenoff, Adv. Funct. Mater., 2012, 22, 1923-1931.

195 K. Kelly and J. Schlenoff, ACS Appl. Mater. Interfaces, 2015, 7, 13980-13986.

196 I. Kurtz, S. Sui, X. Hao, M. Huang, S. Perry and J. Schiffman, ACS Appl. Bio Mater., 2019, 2, 3926-3933.

197 F. Zhu, L. Cheng, J. Yin, W. Z.L., J. Qian, F. J. and Q. Zheng, ACS Appl. Mater. Interfaces, 2016, 8, 31304-31310.

198 X. Meng, S. Perry and J. Schiffman, ACS Macro Letters, 2017, 
6, 505-511.

199 X. Meng, J. Schiffman and S. Perry, Macromolecules, 2018, 51, 8821-8832.

200 P. Suarez-Martinez, P. Batys, M. Sammalkorpi and J. Lutkenhaus, Macromolecules, 2019, 52, 3066-3074.

201 M. Rubinstein and A. Semenov, Macromolecules, 2001, 34, 1058-1068.

202 M. Andreev, V. M. Prabhu, J. F. Douglas, M. V. Tirrell and J. J. de Pablo, Macromolecules, 2018, 51, 6717-6723.

203 Y. Zhang, F. Li, L. D. Valenzuela, M. Sammalkorpi and J. L. Lutkenhaus, Macromolecules, 2016, 49, 7563-7570.

204 R. Zhang, Y. Zhang, H. Antila, J. L. Lutkenhaus and M. Sammalkorpi, J. Phys. Chem. B, 2017, 121, 322-333.

205 O’Neal, J.T., K. Wilcox, Y. Zhang, I. George and J. Lutkenhaus, J. Chem. Phys., 2018, 149, 163317.

206 Y. Zhang, P. Batys, J. O’Neal, F. Li, M. Sammalkorpi and J. Lutkenhaus, ACS Cent. Sci., 2018, 4, 638-644.

207 E. Yildirim, Y. Zhang, J. L. Lutkenhaus and M. Sammalkorpi, ACS Macro Lett., 2015, 4, 1017-1021.

208 A. Vidyasagar, C. Sung, R. Gamble and J. L. Lutkenhaus, ACS Nano, 2012, 6, 6174-6184.

209 A. Vidyasagar, C. Sung, K. Losensky and J. L. Lutkenhaus, Macromolecules, 2012, 45, 9169-9176.

210 C. Sung, K. Hearn and J. Lutkenhaus, Soft Matter, 2014, 10, 6467-6476.

211 F. S. Bates, Science, 1991, 251, 898-905.

212 C. M. Bates and F. S. Bates, Macromolecules, 2017, 50, 3-22.

213 Y. Yan, A. de Keizer, M. A. Cohen Stuart, M. Drechsler and N. A. M. Besseling, J. Phys. Chem. B, 2008, 112, 1090810914.

214 H. M. van der Kooij, E. Spruijt, I. K. Voets, R. G. Fokkink, M. A. Cohen Stuart and J. van der Gucht, Langmuir, 2012, 28, 14180-14191.

215 W. Blocher and S. Perry, WIREs Nanomed. Nanobiotech., 2017, 9, e1442.

216 M. A. Cohen Stuart, N. A. M. Besseling and R. G. Fokkink, Langmuir, 2004, 20, 2785-2791.

217 E. Y. Kramarenko, A. Khokhlov and P. Reineker, J. Chem. Phys., 2006, 125, 194902.

218 M. Cohen Stuart, B. Hofs and d. K. A. Voets, I.K., Curr. Opin. Colloid Interface Sci., 2005, 10, 30-36.

219 B. Hofs, A. de Keizer and M. Cohen Stuart, J. Phys. Chem. B, 2007, 111, 5621-5627.

220 H. Dautzenberg, Macromol. Chem. Phys., 2000, 201, 17651773.

221 S. Holappa, L. Kantonen, T. Andersson, F. Winnik and H. Tenhu, Langmuir, 2005, 21, 11431-11438.

222 J.-F. Gohy, S. K. Varshney, S. Antoun and R. Jerome, Macromolecules, 2000, 33, 9298-9305.

223 B. Hofs, I. Voets, A. de Keizer and M. Cohen Stuart, Phys. Chem. Chem. Phys., 2006, 8, 4242-4251.

224 D. V. Pergushov, A. H. E. MÃijller and F. H. Schacher, Chem. Soc. Rev., 2012, 41, 6888-6901.

225 J. N. Hunt, K. E. Feldman, N. A. Lynd, J. Deek, L. M. Campos,
J. M. Spruell, B. M. Hernandez and E. J. Kramer, Adv. Mater., 2011, 23, 2327-2331.

226 G. M. C. Ong and C. E. Sing, Soft Matter, 2019, 15, 51165127.

227 F. Bossard, V. Sfika and C. Tsitsilianis, Macromolecules, 2004, 37, 3899-3904.

228 H. Cui, X. Zhuang, C. He., Y. Wei and X. Chen, Acta Biomaterialia, 2015, 11, 183-190.

229 M. Lemmers, I. Voets, M. Cohen Stuart and J. van der Gucht, Soft Matter, 2011, 7, 1378-1389.

230 M. Lemmers, J. Sprakel, I. Voets, J. van der Gucht and M. Cohen Stuart, Angew. Chem. Int. Ed., 2010, 49, 708-711.

231 T. P. Lodge, K. J. Hanley, B. Pudil and V. Alahapperuma, Macromolecules, 2003, 36, 816-822.

232 T. P. Lodge, B. Pudil and K. J. Hanley, Macromolecules, 2002, 35, 4707-4717.

233 C.-I. Huang and T. P. Lodge, Macromolecules, 1998, 31, 3556-3565.

234 C. Lai, W. B. Russel and R. A. Register, Macromolecules, 2002, 35, 841-849.

235 C. E. Sing, J. Chem. Phys., 2020, 152, DOI: 10.1063/1.5140756.

236 G. Decher and J. Hong, Makromol. Chem., Macromol. Symp., 1991, 46, 321-327.

237 G. Decher and J. Hong, Ber Bunsen Phys Chem, 1991, 95, 1430-1434.

238 G. Decher, Science, 1997, 277, 1232-1237.

239 D. Kovacevic, S. van der Burgh, A. de Keizer and M. Cohen Stuart, Langmuir, 2002, 18, 5607-5612.

240 S. Lindhoud and M. Cohen Stuart, Adv. Polym. Sci., 2014, 255, 139-172.

241 A. Salehi, P. Desai, J. Li, C. Steele and R. G. Larson, Macromolecules, 2015, 48, 400-409.

242 C. P. Brangwynne, C. Eckmann, D. Courson, A. Rybarska, C. Hoege, J. Gharakhani, F. Jülicher and A. A. Hyman, Science, 2009, 324, 1729-1732.

243 R. Narayanaswamy, M. Levy, M. Tsechansky, G. Stovall, J. O'Connell, J. Mirrielees, A. Ellington and E. Marcotte, Proc. Natl. Acad. Sci. U. S. A., 2009, 106, 10147-10152.

244 C. P. Brangwynne, T. J. Mitchison and A. A. Hyman, Proc. Natl. Acad. Sci. U. S. A., 2011, 108, 4334-4339.

245 A. A. Hyman and K. Simons, Science, 2012, 337, 1047-1049.

246 A. A. Hyman, C. A. Weber and F. Jülicher, Annu. Rev. Cell Dev. Biol., 2014, 40, 39-58.

247 S. C. Weber and C. P. Brangwynne, Cell, 2012, 149, 11881191.

248 S. C. Weber, Curr. Opin. Cell Bio., 2017, 46, 62-71.

249 L. Zhu and C. P. Brangwynne, Curr. Opin. Cell Biol., 2015, 34, 23-30.

250 J. Werner, E. Chen, J. Guberman, A. Zippilli, J. Irgon and Z. Gitai, Proc. Natl. Acad. Sci. U. S. A., 2009, 106, 78587863.

251 C. Bayas, J. Wang, M. Lee, J. Schrader, L. Shapro and W. Mo- 
erner, Proc. Natl. Acad. Sci. U. S. A., 2018, 115, E3712E3721.

252 S. Perry and C. Sing, ACS Macro Lett., 2020, 9, 216-225.

253 S. F. Banani, H. O. Lee, A. A. Hyman and M. K. Rosen, Nat. Rev. Mol. Cell Biol., 2017, 18, 285-298.

254 C. Lee, C. P. Brangwynne, J. Gharakhani, A. Hyman and F. Jülicher, Phys. Rev. Lett., 2013, 111, 088101.

255 A. Rai, J.-X. Chen, M. Selbach and L. Pelkmans, Nature, 2018, 559, 211-216.

256 S. Saha, C. Weber, M. Nousch, O. Adame-Arana, C. Hoerge, M. Hein, E. Osborne-Nishimura, J. Mahamid, M. Jahnel, L. Jawerth, A. Pozniakovski, C. Eckmann, F. Jülicher and A. A. Hyman, Cell, 2016, 166, 1572-1584.

257 B. Schuster, E. Reed, R. Parthasarathy, C. Jahnke, R. Caldwell, J. Bermudez, H. Ramage, M. Good and D. Hammer, Nat. Commun., 2018, 9, 2985.

258 Y.-H. Lin, J. Song, J. Forman-Kay and H. Chan, J. Mol. Liq., 2017, 273, 176-193.

259 S. Das, A. N. Amin, Y.-H. Lin and H. S. Chan, Phys. Chem. Chem. Phys., 2018, 20, 28558.

260 G. L. Dignon, W. Zheng, R. B. Best, Y. C. Kim and J. Mittal, Proc. Natl. Acad. Sci. USA, 2018, 115, 9929-9934.

261 A. Statt, H. Casademunt, C. P. Brangwynne and A. Z. Panagiotopoulos, Model for disordered proteins with strongly sequence-dependent liquid phase behavior, 2019.

262 R. K. Das and R. V. Pappu, Proc. Natl. Acad. Sci. U. S. A., 2013, 110, 13392-13397.

263 Y.-H. Lin, J. D. Forman-Kay and H. S. Chan, Phys. Rev. Lett., 2016, 117, 178101, year.

264 Y. H. Lin and H. S. Chan, Biophys. J., 2017, 112, 2043-2046.

265 R. Das, R. K.M. and R. Pappu, Curr. Opin. in Struct. Bio., 2015, 32, 102-112.

266 L. Sawle and K. Ghosh, J. Chem. Phys., 2015, 143, 085101.

267 V. Sethuraman, M. McGovern, D. C. Morse and K. D. Dorfman, Soft Matter, 2019, 15, 5431-5442.

268 F. S. Bates, M. A. Hillmyer, T. P. Lodge, C. M. Bates, K. T. Delaney and G. H. Fredrickson, Science, 2012, 336, 434440.

269 P. Hodrokoukes, G. Floudas, S. Pispas and N. Hadjichristidis, Macromolecules, 2001, 34, 650-657.

270 M. E. Seitz, C. D. Chan, K. L. Opper, T. W. Baughman, K. B. Wagener and K. I. Winey, J. Am. Chem. Soc., 2010, 132, 8165-8174.

271 L. M. Hall, M. E. Seitz, K. I. Winey, K. L. Opper, K. B. Wagener, M. J. Stevens and A. L. Friscknecht, J. Am. Chem. Soc., 2012, 134, 574-587.

272 J. Jeon and A. V. Dobrynin, J. Phys. Chem. B, 2006, 110, 24652-24665.

273 J. Jeon and A. V. Dobrynin, Macromolecules, 2005, 38, 53005312.

274 J. Jeon and A. V. Dobrynin, Phys. Rev. E, 2003, 67, 061803.

275 K. Q. Hoffmann, S. L. Perry, L. Leon, D. Priftis, M. Tirrell and J. J. de Pablo, Soft Matter, 2015, 11, 1525-1538.

276 M. Rubinstein and A. Dobrynin, Trends in Polymer Science,
1997, 5, 181-186.

277 A. N. Semenov and M. Rubinstein, Macromolecules, 1998, 31, 1373-1385.

278 S. Danielsen, T. Nguyen, G. Fredrickson and R. Segalman, ACS Macro Lett., 2019, 8, 88-94.

279 W. Hollingsworth, C. Segura, J. Balderrama, N. Lopez, P. Schleissner and A. Ayzner, J. Phys. Chem. B, 2016, 120, 7767-7774.

280 M. Record, T. Lohman and P. de Hasketh, J. Mol. Biol., 1976, 107, 145-158.

281 G. Manning, Q. Rev. Biophys., 1978, 11, 179-246.

282 D. Mascotti and T. Lohman, Proc. Natl. Acad. Sci. U. S. A., 1990, 87, 3142-3146.

283 S. Yu, X. Xu, C. Yigit, M. van der Geit, W. Zidek, J. Jankowski, J. Dzubiella and M. Ballauf, Soft Matter, 2015, 11, 46304639.

284 P. L. Dubin, J. Gao and K. Mattison, Sep. Purif. Methods, 1994, 21, 1-16.

285 A. B. Kayitmazer, D. Seeman, B. B. Minsky, P. L. Dubin and Y. Xu, Soft Matter, 2013, 9, 2553-2583.

286 A. Obermeyer, C. Mills, X. Dong, R. Flores and B. Olsen, Soft Matter, 2016, 12, 3570-3581.

287 S. Lindhoud and M. Claessens, Soft Matter, 2016, 12, 408413.

288 C. S. Cummings and A. C. Obermeyer, Biochemistry, 2018, 57, 314-323.

289 J. van Lente and L. S. Claessens, M.M.A.E., Biomacromolecules, 2019, 20, 3696-3703.

290 M. Zhao and N. Zacharia, J. Chem. Phys., 2018, 149, 163326.

291 W. Blocher McTigue and S. Perry, Soft Matter, 2019, 15, 3089-3103.

292 A. Harada and K. Kataoka, J. Am. Chem. Soc., 1999, 121, 9241-9242.

293 M. Giannotti, I. Abasolo, M. Oliva, F. Andrade, N. GarciaAranda, M. Melgarejo, D. Pulido, J. Corchero, Y. Fernandez, A. Villaverde, M. Royo, M. Garcia-Parajo, F. Sanz and S. Schwartz, ACS Appl. Mater. Interfaces, 2016, 8, 2574125752.

294 A. Kishimura, A. Koide, K. Osada, Y. Yamasaki and K. Kataoka, Angew. Chem. Int. Ed., 2007, 46, 6085-6088.

295 S. Lindhoud, L. Voorhaar, R. de Vries, R. Schweins, M. Cohen Stuart and W. Norde, Langmuir, 2009, 25, 408-413.

296 S. Lindhoud, R. de Vries, R. Schweins, M. Cohen Stuart and W. Norde, Soft Matter, 2009, 5, 242-250.

297 S. Lindhoud, R. de Vries, R. Schweins, M. Cohen Stuart and W. Norde, Biomacromolecules, 2007, 8, 2219-2227.

298 A. Nolles, A. Westphal, J. de Hoop, R. Fokkink, J. Kleijn, W. van Berkel and J. Borst, Biomacromolecules, 2015, 16, 1542-1549.

299 N. Pippa, M. Karayianni, S. Pispas and C. Demetzos, Int. J. Pharm., 2015, 491, 136-143.

300 N. Pippa, R. Kalinova, I. Dimitrov, S. Pispas and C. Demetzos, J. Phys. Chem. B, 2015, 119, 6813-6819. 
301 Y. Zhang, K. Han, D. Lu and Z. Liu, Soft Matter, 2013, 9, 8723-8729.

302 R. Samanta and V. Ganesan, Soft Matter, 2018, 14, 94759488.

303 G. Pandav, V. Pryamitsyn, J. Errington and V. Ganesan, J. Phys. Chem. B, 2015, 119, 14536-14550.

304 V. Pryamitsyn and V. Ganesan, J. Chem. Phys., 2015, 143, 164904.

305 V. Pryamitsyn and V. Ganesan, J. Chem. Phys., 2015, 143, 164904.

306 A. Salis and B. W. Ninham, Chem. Soc. Rev., 2014, 43, 73587377.

307 C. Wang, C.-K. D. Ma, H. Yeon, X. Wang, S. Gellman and N. Abbott, J. Am. Chem. Soc., 2017, 51, 18536-18544.

308 H. Yeon, C. Wang, R. van Lehn and N. Abbott, Langmuir, 2017, 33, 4628-4637.

309 L. Leibler, M. Rubinstein and R. Colby, Macromolecules, 1991, 24, 4701-4707.

310 D. Ermak and J. McCammon, J. Chem. Phys., 1978, 69, 1352-1360.

311 P. Rouse, J. Chem. Phys., 1953, 21, 1272-1280.

312 R. Larson, J. Rheol., 2005, 49, 1-70.

313 B. Zimm, J. Chem. Phys., 1956, 24, 269-278.

314 C. Schroeder, J. Rheol., 2018, 62, 371-403.

315 C. Stoltz, J. de Pablo and M. Graham, J. Rheol., 2006, 50, 137-167.

316 C.-C. Huang, R. Winkler, G. Sutmann and G. Gompper, Macromolecules, 2010, 43, 10107-10116.
317 C. Sasmal, K.-W. Hsiao, C. Schroeder and J. Ravi Prakash, J. Rheol., 2017, 61, 169-186.

318 C. Young and C. E. Sing, J. Chem. Phys., 2019, 151, 124907.

319 A. Saadat and B. Khomami, Phys. Rev. E, 2015, 92, 033307.

320 M. Muthukumar, Proc. Nat. Acad. Sci. USA, 2016, 113, 12627-12632.

321 M. Muthukumar, J. Chem. Phys., 1997, 107, 2619-2635.

322 Y. Wang, K. Kimura, Q. Huang and P. L. Dubin, Macromolecules, 1999, 32, 7128-7134.

323 Y. Fan, M. Kellermeier, A. Xu, B. Boyko, S. Mirtschin and P. L. Dubin, Macromolecules, 2017, 50, 5518-5526.

324 F. Comert, A. Xu, V. Madro, S.P. Liadinskaia and P. L. Dubin, J. Chem. Phys., 2018, 149, 163321.

325 F. Karimi, N. Qazvini and R. Namivandi-Zangeneh, Int. J. Biol. Macromol., 2013, 61, 102-113.

326 B. Jing, J. Qiu and Y. Zhu, Soft Matter, 2017, 13, 4881-4889.

327 V. Pryamitsyn and V. Ganesan, Macromolecules, 2014, 47, 6095-6112.

328 R. Messina, C. Holm and K. Kremer, J. Polym. Sci. Part B: Polym. Phys., 2004, 42, 3557-3570.

329 C. Tong, J. Chem. Phys., 2013, 139, 084903.

330 T. Wallin and P. Linse, Langmuir, 1996, 12, 305-314.

331 J. Koski, H. Chao and R. Riggleman, J. Chem. Phys., 2013, 139, 244911.

332 N. Nair and A. Jayaraman, Macromolecules, 2010, 43, 82518263.

333 T. Martin, P. Dodd and A. Jayaraman, Phys. Rev. Lett., 2013, 110, 018301. 